\title{
Control of Diastereoselectivity in Tandem Asymmetric Reactions Generating Nonadjacent Stereocenters with Bifunctional Catalysis by Cinchona Alkaloids
}

\author{
Baomin Wang, Fanghui Wu, Yi Wang, Xiaofeng Liu and Li Deng* \\ Department of Chemistry \\ Brandeis University \\ Waltham, Massachusetts 02454-9110
}

\section{Supporting Information}

General Information. ${ }^{1} \mathrm{H}$ and ${ }^{13} \mathrm{C}$ NMR spectra were recorded on a Varian instrument (400 $\mathrm{MHz}$ and $100 \mathrm{MHz}$, respectively) and internally referenced to tetramethylsilane signal or residual protio solvent signals. Data for ${ }^{1} \mathrm{H}$ NMR are recorded as follows: chemical shift $(\delta, \mathrm{ppm})$, multiplicity (s, singlet; $\mathrm{d}$, doublet; t, triplet; $\mathrm{q}$, quartet; $\mathrm{m}$, multiplet), integration, coupling constant $(\mathrm{Hz})$. Data for ${ }^{13} \mathrm{C} N M R$ are reported in terms of chemical shift $(\delta, \mathrm{ppm})$. Infrared spectra were recorded on a Perkin Elmer FT-IR Spectrometer and are reported in frequency of absorption. Low resolution and high resolution mass spectra were recorded on either a Micromass 70-VSE-B instrument (EI, CI) or a Micromass Q-Tof instrument (ESI). Specific rotations were measured on a Jasco Digital Polarimeter.

High performance liquid chromatography (HPLC) analysis was performed on a HewlettPackard 1100 Series instrument equipped with a quaternary pump, using a Daicel Chiralcel OJ, OD Column ( 250 x $4.6 \mathrm{~mm})$ or Chiralpak AD, AS Column $(250 \times 4.6 \mathrm{~mm})$. UV absorption was monitored at $220 \mathrm{~nm}, 254 \mathrm{~nm}$ or $280 \mathrm{~nm}$. Chiral GC was performed on Hewlett-Packard 6890 GC system, using HP chiral (20\% Permethylated $\beta$ Cyclodextrin) column. 
Materials: 3a, ${ }^{1} \mathbf{3 b},{ }^{2} \mathbf{3 c},{ }^{3} \mathbf{3 d},{ }^{4} \mathbf{3 f}-\mathbf{k},{ }^{5} 3 \mathbf{3},{ }^{6} \mathbf{3} \mathbf{m},{ }^{7}$ were prepared according to literature methods. 3e was purchased from Alfa Aesar and used without purification. $\alpha$ Chloroacrylonitrile 4 and acrylonitrile 6 were purchased from Aldrich and used without further purification. (Caution: Acrylonitrile 6 is a regulated carcinogen and should be used with careful precaution in hood.)

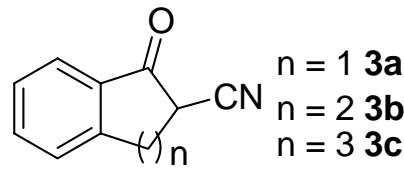<smiles>CC(=O)C(C)C#N</smiles>

3)<smiles>C=CCC(C#N)C(=O)OC</smiles>

$3 m$<smiles>N#CC1[Ge]CCCC1=O</smiles>

$$
\begin{aligned}
& \mathrm{Ar}=\mathrm{Ph}-\quad \mathbf{3 e} \\
& \text { 4-F-Ph- } \quad 3 f \\
& \text { 4-Cl-Ph- } \quad 3 g \\
& \text { 4-Br-Ph- } \quad 3 h \\
& \text { 4-Me-Ph- 3i } \\
& \text { 4-MeoPh- 3j } \\
& \text { 2-naphthyl- } \mathbf{3 k}
\end{aligned}
$$

Quinine, quinidine, and cinchonidine were purchased from Aldrich Inc. and used without further purification. C9-epi-Q was prepared according to literature procedure. ${ }^{8} \mathrm{Q}-\mathbf{1 a}$, QD1a, Q-1b, and QD-1b were prepared according to our reported methods. ${ }^{9}$ Q-2a, Q-2b, Q$\mathbf{2 c}$, QD-2c were prepared according to literature procedures. ${ }^{10}$<smiles>C=C1C2CCN1C([C@H](O)c1ccnc3ccc(OC)cc13)C2C</smiles>

Quinine<smiles>C=CC1C2CCN1N(C)C2[C@H](O)c1ccnc2ccc(OC)cc12</smiles>
C9-epi-Q<smiles>O[C@H](c1ccnc2ccccc12)C1CC2C=CC1C2</smiles>

Cinchonidine<smiles>C=CC1CN2CCC1C2[C@H](O)c1ccnc2ccc(OC)cc12</smiles>

Quinidine<smiles>O=C1CC2CCN1CC2[C@H](O)c1ccnc2ccc(O)cc12</smiles>

QD-1

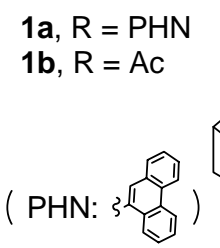

(5)

Q-1<smiles>C=CC1C2CCN1C([C@H](NC(=S)NC)c1ccnc3ccc(OC)cc13)C2</smiles>

QD-2<smiles>COc1ccc2nccc([C@H](NC(=S)NC3CCCCC3)C3C4CCC3CC4)c2c1</smiles>

$2 \mathbf{a} \mathrm{Ar}=\mathrm{Ph}-; \quad \mathbf{2 b} \mathrm{Ar}=4-t-\mathrm{Bu}-\mathrm{Ph}-;$

2c Ar $=3,5-b i s \mathrm{CF}_{3} \mathrm{Ph}-$ 
S-Table 1. Asymmetric Conjugate Addition-Protonation of Michael donors (3a-e, 3h, 3l) with 2-Chloroacrylonitrile catalyzed by catalysts $2 \mathrm{c}$ and $\mathbf{1}$.

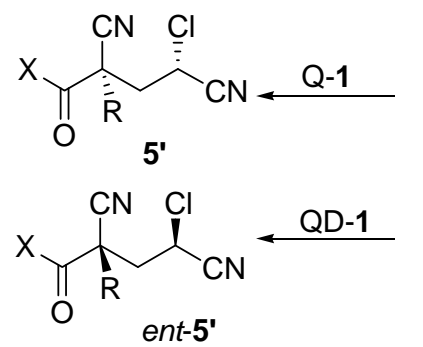

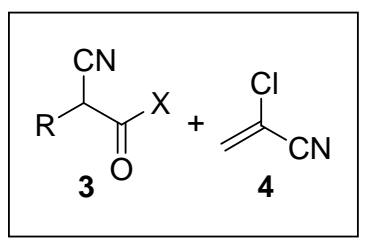

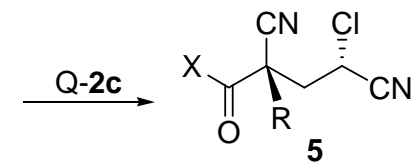

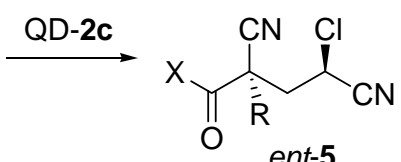

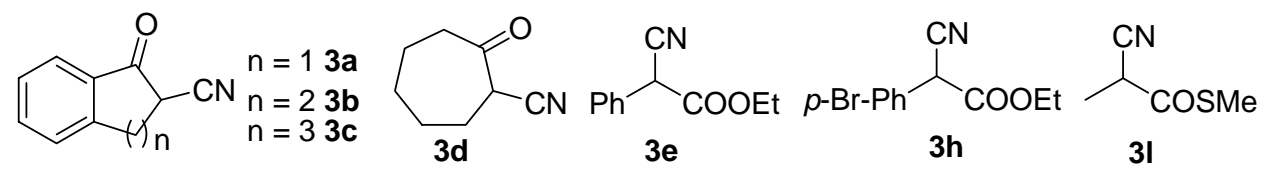

\begin{tabular}{|c|c|c|c|c|c|c|c|c|c|c|c|c|c|}
\hline \multirow{2}{*}{3} & \multirow{2}{*}{ cat. } & \multicolumn{3}{|c|}{5} & \multicolumn{3}{|c|}{ ent -5} & \multicolumn{3}{|c|}{$5^{\prime}$} & \multicolumn{3}{|c|}{ ent-5' } \\
\hline & & $\mathrm{dr}$ & ee/\% & yield $/ \%$ & $\mathrm{dr}$ & ee/\% & yield/\% & $d r$ & ee $/ \%$ & yield/\% & $\mathrm{dr}$ & ee/\% & yield/\% \\
\hline \multirow{3}{*}{$3 a$} & \multirow{3}{*}{$\begin{array}{l}\text { Q-2c } \\
\text { QD-2c } \\
\text { Q-1a } \\
\text { QD-1a }\end{array}$} & \multirow[t]{3}{*}{ 10:1 } & \multirow[t]{3}{*}{97} & \multirow[t]{3}{*}{99} & \multirow{3}{*}{$9: 1$} & \multirow{3}{*}{97} & \multirow{3}{*}{99} & & & & & & \\
\hline & & & & & & & & $20: 1$ & 93 & $\left.95\right|^{a}$ & & & \\
\hline & & & & & & & & & & & $20: 1$ & 96 & $95]^{a}$ \\
\hline \multirow{3}{*}{$3 b$} & \multirow{3}{*}{$\begin{array}{l}\text { Q-2c } \\
\text { QD-2c } \\
\text { Q-1a } \\
\text { QD-1a }\end{array}$} & \multirow[t]{3}{*}{$16: 1$} & \multirow[t]{3}{*}{99} & \multirow[t]{3}{*}{94} & \multirow{3}{*}{$12: 1$} & \multirow{3}{*}{97} & \multirow{3}{*}{98} & & & & & & \\
\hline & & & & & & & & $8: 1$ & 87 & $92^{a}$ & & & \\
\hline & & & & & & & & & & & 11:1 & 91 & $87^{d}$ \\
\hline \multirow{3}{*}{$3 c$} & \multirow{3}{*}{$\begin{array}{l}\text { Q-2c } \\
\text { QD-2c } \\
\text { Q-1a } \\
\text { QD-1a }\end{array}$} & \multirow[t]{3}{*}{$9: 1$} & \multirow[t]{3}{*}{97} & \multirow[t]{3}{*}{98} & \multirow{3}{*}{$9: 1$} & \multirow{3}{*}{96} & \multirow{3}{*}{96} & \multirow{3}{*}{$25: 1$} & & & & & \\
\hline & & & & & & & & & 94 & $92^{a}$ & & & \\
\hline & & & & & & & & & & & $25: 1$ & 95 & $\left.88\right|^{a}$ \\
\hline & Q-2c & 14:1 & 94 & 98 & $10 \cdot 1$ & 98 & 97 & & & & & & \\
\hline 3d & Q-1a & & & & & & & $5: 1$ & 85 & 92 & & & \\
\hline & QD-1a & & & & & & & & & & $7: 1$ & 91 & 87 \\
\hline & Q-2c & $15: 1$ & 95 & 98 & & & & & & & & & \\
\hline $3 e$ & $\begin{array}{l}\text { QD-2c } \\
\text { Q-1b }\end{array}$ & & & & 16:1 & 95 & 99 & $5: 1$ & 86 & 45 & & & \\
\hline & QD-1b & & & & & & & & & & $4: 1$ & 88 & $\left.85\right|^{d}$ \\
\hline & Q-2c & $12: 1$ & 95 & 98 & & & & & & & & & \\
\hline $3 h$ & $0-1 \mathrm{~h}$ & & & & 12.1 & 94 & 90 & $2 \cdot 1$ & 80 & 75 & & & \\
\hline & QD-1b & & & & & & & & & & $4: 1$ & 86 & 98 \\
\hline & Q-2c & $9: 1$ & 96 & 98 & & & & & & & & & \\
\hline 31 & $\begin{array}{l}\text { QD-2c } \\
\text { O-1b }\end{array}$ & & & & 9:1 & 96 & 98 & $7 \cdot 1$ & 88 & 44 & & & \\
\hline & QD-1b & & & & & & & & & & $10: 1$ & 93 & 71 \\
\hline
\end{tabular}

${ }^{a}$ These data were documented in our previous report. ${ }^{11}$ 


\section{General procedure for the asymmetric conjugate addition of $\alpha$-cyano carbonyl compounds to acrylonitrile or $\alpha$-chloroacrylonitrile}

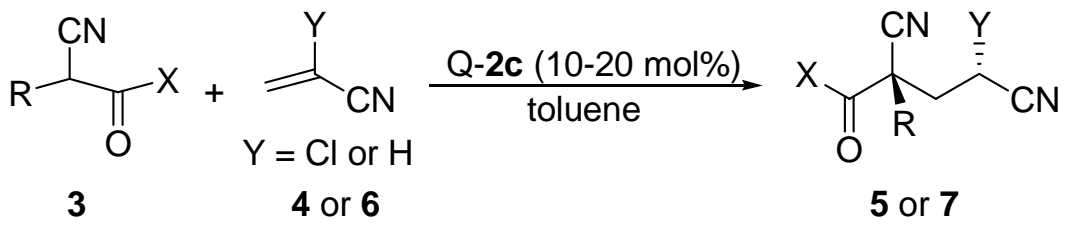

To a solution of $\alpha$-cyano carbonyl compound $3(0.2 \mathrm{mmol}$ or $0.3 \mathrm{mmol})$ and catalyst Q2c $(10-20 \mathrm{~mol} \%)$ in toluene $(2.0 \mathrm{~mL}$ or $3.0 \mathrm{~mL})$ was added $\alpha$-chloroacrylonitrile 4 (3.0 equiv.) or acrylonitrile 6 (3.0 equiv). The reaction mixture was kept at room temperature until $\alpha$-cyano carbonyl compound 3 was completely consumed. The crude reaction mixture was filtered through a short plug of silica gel for the removal of the catalyst and washed with diethyl ether $(5.0 \mathrm{~mL})$. The filtrate was concentrated in vacuo, and the residue was subjected to flash chromatography on silica gel to give the pure adduct 5 or 7.

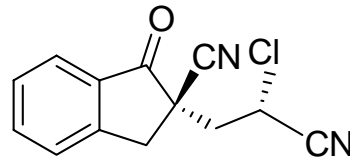

$5 a$

Q-2c (12.0 mg, $10 \mathrm{~mol} \%)$ catalyzed reaction of 3a (31.4 mg, 0.2 $\mathrm{mmol})$ and $4(52.5 \mathrm{mg}, 0.6 \mathrm{mmol})$ in toluene $(2.0 \mathrm{~mL})$ was run at room temperature for $1 \mathrm{~h}$ to furnish the crude product $[\mathrm{dr}=10: 1$, determined by ${ }^{1} \mathrm{H}$ NMR analysis of the crude product]. The crude product was purified by flash chromatography on silica gel (hexanes/ethyl acetate $=3 / 1$ ) to give adducts $\mathbf{5 a}$ and 5'a $(\mathrm{dr}=10: 1)$ as a white solid in $99 \%$ yield. The ee of the major diastereomer was determined to be $97 \%$ ee [determined by HPLC, Chiralpak $\mathrm{AD} \times 2$, hexanes: isopropanol $=80: 20,1.0 \mathrm{~mL} / \mathrm{min}, \lambda 220 \mathrm{~nm}, \mathrm{t}$ (major) $=18.16 \mathrm{~min}, \mathrm{t}($ minor $)=23.75 \mathrm{~min}] .{ }^{1} \mathrm{H} \mathrm{NMR}$ $\left(400 \mathrm{MHz}, \mathrm{CDCl}_{3}\right) \delta 7.87(\mathrm{~d}, J=8.0 \mathrm{~Hz}, 1 \mathrm{H}), 7.78(\mathrm{t}, J=7.6 \mathrm{~Hz}, 1 \mathrm{H}), 7.57-7.51(\mathrm{~m}$, 2H), 4.93 (dd, $J=5.2 \mathrm{~Hz}, 10.4 \mathrm{~Hz}, 1 \mathrm{H}$ ), 3.83 (d, $J=17.6 \mathrm{~Hz}, 1 \mathrm{H}), 3.63$ (d, $J=17.6 \mathrm{~Hz}$, $1 \mathrm{H}), 2.87(\mathrm{dd}, J=5.2 \mathrm{~Hz}, 14.8 \mathrm{~Hz}, 1 \mathrm{H}), 2.45(\mathrm{dd}, J=10.4 \mathrm{~Hz}, 14.0 \mathrm{~Hz}, 1 \mathrm{H}) ;{ }^{13} \mathrm{C} \mathrm{NMR}$ $\left(100 \mathrm{MHz}, \mathrm{CDCl}_{3}\right) \delta 195.0,150.4,137.3,132.1,129.2,126.8,126.0,117.3,116.2,45.9$, 41.4, 38.4; HRMS (EI/[M $\left.{ }^{+}\right]$) m/z calcd. for $\mathrm{C}_{13} \mathrm{H}_{9} \mathrm{~N}_{2} \mathrm{OCl}$ : 244.0403, found: 244.0400; IR (neat) $\vee 1717 \mathrm{~cm}^{-1}$. 
ent-5a was obtained as a white solid in $99 \%$ yield and $97 \%$ ee with 9:1 dr from a reaction catalyzed by QD-2c (10 mol\%) at room temperature for $1 \mathrm{~h}$.

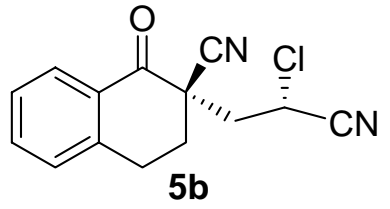

Q-2c (18.0 mg, $10 \mathrm{~mol} \%)$ catalyzed reaction of $\mathbf{3 b}(51.3 \mathrm{mg}, 0.3$ $\mathrm{mmol})$ and $4(78.8 \mathrm{mg}, 0.9 \mathrm{mmol})$ in toluene $(3.0 \mathrm{~mL})$ was run at room temperature for $11 \mathrm{~h}$ to furnish the crude product $[\mathrm{dr}=$ 16:1, determined by ${ }^{1} \mathrm{H}$ NMR analysis of the crude product]. The crude product was purified by flash chromatography on silica gel (hexanes/ethyl acetate $=3 / 1$ ) to give adducts $\mathbf{5 b}$ and $\mathbf{5} \mathbf{b}(\mathrm{dr}=17: 1)$ as a colorless oil in $94 \%$ yield. The ee of the major diastereomer was determined to be $99 \%$ ee [determined by HPLC, (R, R)-Whelk-O 1, hexanes: isopropanol $=98: 2,0.6 \mathrm{~mL} / \mathrm{min}, \lambda 220 \mathrm{~nm}, \mathrm{t}($ major $)=60.28 \mathrm{~min}, \mathrm{t}($ minor $)=$ $46.04 \mathrm{~min}] .{ }^{1} \mathrm{H}$ NMR (400 MHz, $\left.\mathrm{CDCl}_{3}\right) \delta 8.03(\mathrm{~d}, J=7.6 \mathrm{~Hz}, 1 \mathrm{H}), 7.61(\mathrm{t}, J=7.2 \mathrm{~Hz}$, $1 \mathrm{H}), 7.40(\mathrm{t}, J=7.2 \mathrm{~Hz}, 1 \mathrm{H}), 7.31(\mathrm{~d}, J=7.2 \mathrm{~Hz}, 1 \mathrm{H}), 4.92(\mathrm{dd}, J=5.2 \mathrm{~Hz}, 8.4 \mathrm{~Hz}, 1 \mathrm{H})$, 3.45-3.37 (m, 1H), 3.11 (dt, $J=4.4 \mathrm{~Hz}, 17.6 \mathrm{~Hz}, 1 \mathrm{H}), 2.90$ (dd, $J=5.6 \mathrm{~Hz}, 14.8 \mathrm{~Hz}, 1 \mathrm{H}$ ), 2.73-2.63 (m, 2H), 2.38-2.31 (m, 1H); ${ }^{13} \mathrm{C}$ NMR $\left(100 \mathrm{MHz}, \mathrm{CDCl}_{3}\right) \delta 188.3,142.3$,

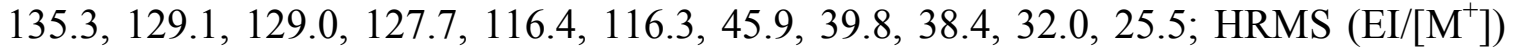
$\mathrm{m} / \mathrm{z}$ calcd. for $\mathrm{C}_{14} \mathrm{H}_{11} \mathrm{~N}_{2} \mathrm{OCl}: 258.0560$, found: 258.0558 ; IR (neat) $v 1700 \mathrm{~cm}^{-1}$.

ent-5b was obtained as a colorless oil in $98 \%$ yield and $97 \%$ ee with $12: 1 \mathrm{dr}$ from a reaction catalyzed by QD-2c (10 mol\%) at room temperature for $11 \mathrm{~h}$.

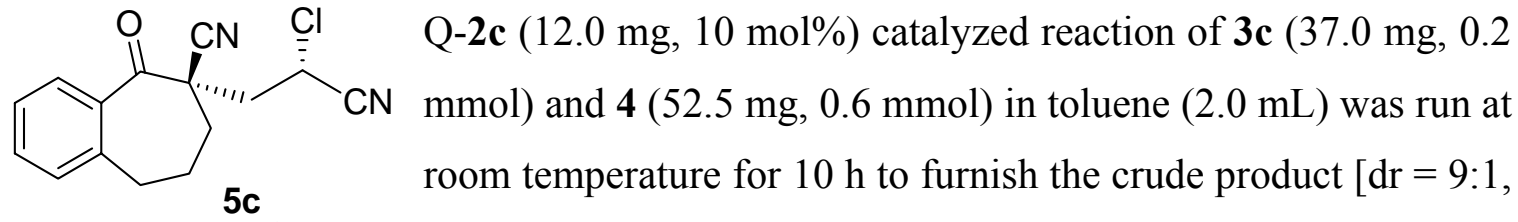
determined by ${ }^{1} \mathrm{H}$ NMR analysis of the crude product]. The crude product was purified by flash chromatography on silica gel (hexanes/ethyl acetate $=3 / 1$ ) to give adducts $\mathbf{5 c}$ and $\mathbf{5} \mathbf{c}(\mathrm{dr}=9: 1)$ as a colorless oil in $98 \%$ yield. The ee of the major diastereomer was determined to be $97 \%$ ee [determined by HPLC, Chiralcel OD, hexanes: isopropanol $=$ 90: 10, $1.0 \mathrm{~mL} / \mathrm{min}, \lambda 220 \mathrm{~nm}, \mathrm{t}$ (major) $=43.67 \mathrm{~min}, \mathrm{t}$ (minor) $=33.04 \mathrm{~min}] .{ }^{1} \mathrm{H} \mathrm{NMR}$ (400 MHz, $\left.\mathrm{CDCl}_{3}\right) \delta$ 7.49-7.25 (m, 4H), $4.76(\mathrm{t}, J=7.2 \mathrm{~Hz}, 1 \mathrm{H}), 3.21(\mathrm{~m}, 1 \mathrm{H}), 3.04$ (dd, $J=7.2 \mathrm{~Hz}, 14.4 \mathrm{~Hz}, 1 \mathrm{H}), 2.95$ (m, 1H), 2.57 (dd, $J=7.2 \mathrm{~Hz}, 14.4 \mathrm{~Hz}, 1 \mathrm{H}), 2.42-2.26$ (m, 
2H), 2.07-1.95 (m, 2H); ${ }^{13} \mathrm{C}$ NMR $\left(100 \mathrm{MHz}, \mathrm{CDCl}_{3}\right) \delta 197.9,138.7,137.2,132.5$, $129.8,128.7,127.1,117.8,116.1,50.8,40.9,38.7,36.3,32.9,24.1 ; \mathrm{HRMS}\left(\mathrm{EI} /\left[\mathrm{M}^{+}\right]\right) \mathrm{m} / \mathrm{z}$ calcd. for $\mathrm{C}_{15} \mathrm{H}_{13} \mathrm{~N}_{2} \mathrm{OCl}$ : 272.0716, found: 272.0714; IR (neat) $v 1700 \mathrm{~cm}^{-1}$.

ent-5c was obtained as a colorless oil in 96\% yield and 96\% ee with 9:1 dr from a reaction catalyzed by QD-2c (10 mol\%) at room temperature for $11 \mathrm{~h}$.

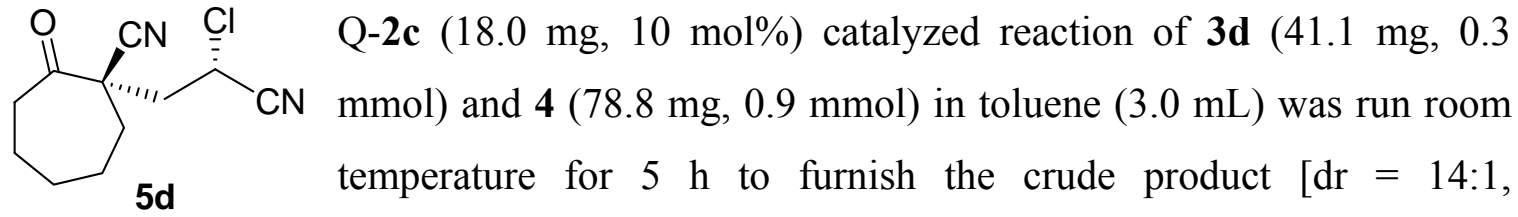
determined by ${ }^{1} \mathrm{H}$ NMR analysis of the crude product]. The crude product was purified by flash chromatography on silica gel (hexanes/ethyl acetate $=4 / 1$ ) to give adducts $\mathbf{5 d}$ and $\mathbf{5} \mathbf{d}(\mathrm{dr}=16: 1)$ as a white solid in $98 \%$ yield. The ee of the major diastereomer was determined to be $94 \%$ ee [determined by GC, $\beta$-ICD, $100{ }^{\circ} \mathrm{C}, 30 \mathrm{~min}$, then $100{ }^{\circ} \mathrm{C}-200^{\circ} \mathrm{C}$ $\left(0.5{ }^{\circ} \mathrm{C} / \mathrm{min}\right), \mathrm{t}$ (major) $=181.60 \mathrm{~min}, \mathrm{t}$ (minor $\left.)=180.52 \mathrm{~min}\right] .{ }^{1} \mathrm{H}$ NMR $(400 \mathrm{MHz}$, $\left.\mathrm{CDCl}_{3}\right) \delta 4.75(\mathrm{dd}, J=6.4 \mathrm{~Hz}, 8.8 \mathrm{~Hz}, 1 \mathrm{H}), 3.00(\mathrm{~m}, 1 \mathrm{H}), 2.93(\mathrm{dd}, J=8.4 \mathrm{~Hz}, 14.4 \mathrm{~Hz}$, 1H), 2.70-2.63 (m, 1H), 2.44 (dd, $J=6.8 \mathrm{~Hz}, 14.8 \mathrm{~Hz}, 1 \mathrm{H}), 2.17$ (dt, $J=2.4 \mathrm{~Hz}, 13.2 \mathrm{~Hz}$, 1H), 1.97-1.88 (m, 4H), 1.83-1.64 (m, 2H), 1.33-1.22 (m, 1H); ${ }^{13} \mathrm{C}$ NMR (100 MHz, $\left.\mathrm{CDCl}_{3}\right) \delta 202.8,118.0,115.9,52.0,41.2,40.7,38.8,36.9,27.1,25.9,22.8$; HRMS $\left(\mathrm{EI} /\left[\mathrm{M}^{+}\right]\right) \mathrm{m} / \mathrm{z}$ calcd. for $\mathrm{C}_{11} \mathrm{H}_{13} \mathrm{~N}_{2} \mathrm{OCl}$ : 224.0716, found: 224.0719; IR (neat) $v 1717 \mathrm{~cm}^{-}$ 1.

ent-5d was obtained as a white solid in $97 \%$ yield and $98 \%$ ee with $10: 1 \mathrm{dr}$ from a reaction catalyzed by QD-2c (10 mol\%) at room temperature for $12 \mathrm{~h}$.

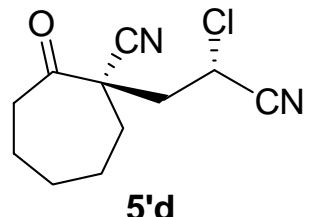

5'd was obtained as a colorless oil in $92 \%$ yield and $85 \%$ ee (The ee was determined by converting $\mathbf{5}$ 'd to compound $\mathbf{8}$ in conc. $\mathrm{HCl}$ at 80 ${ }^{\circ} \mathrm{C}$ for $1 \mathrm{~h}$.) with 5:1 dr from a reaction catalyzed by Q-1a (20 mol\%) at room temperature for $96 \mathrm{~h} .{ }^{1} \mathrm{H} \mathrm{NMR}\left(400 \mathrm{MHz}, \mathrm{CDCl}_{3}\right) \delta 4.72(\mathrm{t}, J$ $=7.2 \mathrm{~Hz}, 1 \mathrm{H}), 3.03(\mathrm{~m}, 1 \mathrm{H}), 2.90(\mathrm{dd}, J=7.2 \mathrm{~Hz}, 14.4 \mathrm{~Hz}, 1 \mathrm{H}), 2.67$ (m, 1H), 2.39 (dd, $J$ $=7.2 \mathrm{~Hz}, 14.8 \mathrm{~Hz}, 1 \mathrm{H}), 2.15(\mathrm{~m}, 1 \mathrm{H}), 1.97(\mathrm{~m}, 4 \mathrm{H}), 1.82(\mathrm{~m}, 1 \mathrm{H}), 1.66(\mathrm{~m}, 1 \mathrm{H}), 1.25(\mathrm{~m}$, 
$1 \mathrm{H}) ;{ }^{13} \mathrm{C}$ NMR $\left(100 \mathrm{MHz}, \mathrm{CDCl}_{3}\right) \delta 203.0,118.3,115.9,51.5,41.6,41.3,38.4,37.6$, 27.1, 26.1, 22.5; HRMS (ESI/[M+H] ${ }^{+}$) m/z calcd. for $\mathrm{C}_{11} \mathrm{H}_{14} \mathrm{~N}_{2} \mathrm{OCl}$ : 225.0795, found: 225.0801; IR (neat) $v 1715 \mathrm{~cm}^{-1}$.

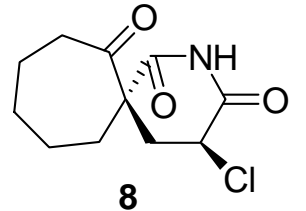

Compound 8. $85 \%$ ee [determined by HPLC, Daicel chiralcel OJ, hexanes: isopropanol $=70: 30,1.0 \mathrm{~mL} / \mathrm{min}, \lambda 220 \mathrm{~nm}, \mathrm{t}$ (major) $=$ $21.14 \mathrm{~min}, \mathrm{t}($ minor $)=25.24 \mathrm{~min}] .{ }^{1} \mathrm{H}$ NMR $\left(400 \mathrm{MHz}, \mathrm{CDCl}_{3}\right) \delta 7.96$ (brs, 1H), 4.59 (dd, $J=5.6 \mathrm{~Hz}, 10.4 \mathrm{~Hz}, 1 \mathrm{H}), 2.93(\mathrm{~m}, 2 \mathrm{H}), 2.71(\mathrm{~m}, 1 \mathrm{H}), 2.37-2.25$ (m, $2 \mathrm{H}), 1.94-1.83$ (m, 3H), 1.73-1.52 (m, 4H); ${ }^{13} \mathrm{C} \mathrm{NMR}\left(100 \mathrm{MHz}, \mathrm{CDCl}_{3}\right) \delta 208.3,172.0$, 166.6, 58.6, 51.3, 41.9, 35.6, 32.7, 30.4, 26.5, 24.7; HRMS (ESI/[M+H] $\left.{ }^{+}\right) \mathrm{m} / \mathrm{z}$ calcd. for $\mathrm{C}_{11} \mathrm{H}_{15} \mathrm{NO}_{3} \mathrm{Cl}: 244.0740$, found: 244.0738; IR (neat) $v 1715,1689 \mathrm{~cm}^{-1}$.

ent-5'd was obtained as a colorless oil in $87 \%$ yield and $91 \%$ ee with $7: 1 \mathrm{dr}$ from a reaction catalyzed by QD-1a (20 mol\%) at room temperature for $96 \mathrm{~h}$.

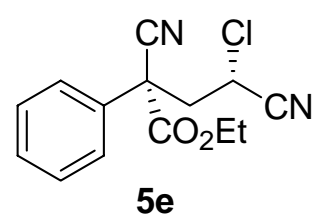

Q-2c $(18.0 \mathrm{mg}, 10 \mathrm{~mol} \%)$ catalyzed reaction of $3 \mathbf{e}(56.7 \mathrm{mg}, 0.3$ $\mathrm{mmol})$ and $4(78.8 \mathrm{mg}, 0.9 \mathrm{mmol})$ in toluene $(3.0 \mathrm{~mL})$ was run at room temperature for $12 \mathrm{~h}$ to furnish the crude product [ $\mathrm{dr}=15: 1$, determined by ${ }^{1} \mathrm{H}$ NMR analysis of the crude product]. The crude product was purified by flash chromatography on silica gel (hexanes/ethyl acetate $=5 / 1$ ) to give adducts $\mathbf{5 e}$ and $\mathbf{5} \mathbf{e}(\mathrm{dr}=17: 1)$ as a colorless oil in $98 \%$ yield. The ee of the major diastereomer was determined to be $95 \%$ ee [determined by HPLC, Chiralcel OD, hexanes: isopropanol $=80: 20,1.0 \mathrm{~mL} / \mathrm{min}, \lambda 220 \mathrm{~nm}, \mathrm{t}$ (major) $=70.07 \mathrm{~min}, \mathrm{t}($ minor $)=$ $12.04 \mathrm{~min}] .{ }^{1} \mathrm{H}$ NMR $\left(400 \mathrm{MHz}, \mathrm{CDCl}_{3}\right) \delta 7.56(\mathrm{~m}, 2 \mathrm{H}), 7.47(\mathrm{~m}, 3 \mathrm{H}), 4.58(\mathrm{dd}, J=5.6$ $\mathrm{Hz}, 8.8 \mathrm{~Hz}, 1 \mathrm{H}), 4.34-4.22(\mathrm{~m}, 2 \mathrm{H}), 3.21$ (dd, $J=9.2 \mathrm{~Hz}, 14.8 \mathrm{~Hz}, 1 \mathrm{H}), 2.96$ (dd, $J=5.6$ $\mathrm{Hz}, 14.8 \mathrm{~Hz}, 1 \mathrm{H}), 1.26$ (t, $J=7.2 \mathrm{~Hz}, 3 \mathrm{H}) ;{ }^{13} \mathrm{C} \mathrm{NMR}\left(100 \mathrm{MHz}, \mathrm{CDCl}_{3}\right) \delta 166.0,132.0$, 130.0, 129.8, 125.9, 116.2, 115.4, 64.2, 51.4, 42.6, 38.8, 13.6; HRMS $\left(E I /\left[\mathrm{M}^{+}\right]\right) \mathrm{m} / \mathrm{z}$ calcd. for $\mathrm{C}_{14} \mathrm{H}_{13} \mathrm{~N}_{2} \mathrm{O}_{2} \mathrm{Cl}$ : 276.0666, found: 276.0670; IR (neat) $v 1734 \mathrm{~cm}^{-1}$.

ent-5e was obtained as a colorless oil in $99 \%$ yield and $95 \%$ ee with $16: 1 \mathrm{dr}$ from a reaction catalyzed by QD-2c (10 mol\%) at room temperature for $11 \mathrm{~h}$. 


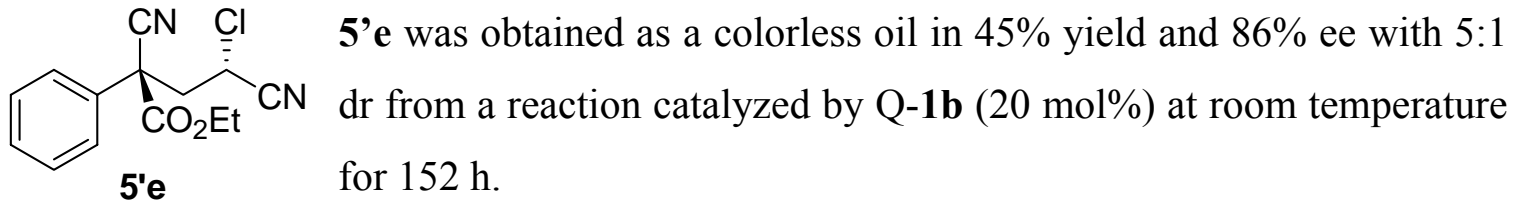<smiles>CCOC(=O)CC(C#N)(C#N)c1ccc(F)cc1</smiles>

Q-2c (18.0 mg, $10 \mathrm{~mol} \%)$ catalyzed reaction of $\mathbf{3 f}(62.1 \mathrm{mg}, 0.3$ $\mathrm{mmol})$ and $4(78.8 \mathrm{mg}, 0.9 \mathrm{mmol})$ in toluene $(3.0 \mathrm{~mL})$ was run at room temperature for $12 \mathrm{~h}$ to furnish the crude product [ $\mathrm{dr}=13: 1$, determined by ${ }^{1} \mathrm{H}$ NMR analysis of the crude product]. The crude product was purified by flash chromatography on silica gel (hexanes/ethyl acetate $=6 / 1$ ) to give adducts $\mathbf{5} \mathbf{f}$ and $\mathbf{5} \mathbf{f}(\mathrm{dr}=17: 1)$ as a colorless oil in $98 \%$ yield. The ee of the major diastereomer was determined to be $95 \%$ ee [determined by HPLC, Chiralcel OD, hexanes: isopropanol $=90: 10,1.0 \mathrm{~mL} / \mathrm{min}, \lambda 220 \mathrm{~nm}, \mathrm{t}($ major $)=18.79 \mathrm{~min}, \mathrm{t}($ minor $)=$ $15.83 \mathrm{~min}] .{ }^{1} \mathrm{H}$ NMR (400 MHz, $\left.\mathrm{CDCl}_{3}\right) \delta 7.55$ (q, 2H), $7.17(\mathrm{t}, J=8.8 \mathrm{~Hz}, 2 \mathrm{H}), 4.61$ (dd, $J=6.0 \mathrm{~Hz}, 8.4 \mathrm{~Hz}, 1 \mathrm{H}), 4.32-4.22(\mathrm{~m}, 2 \mathrm{H}), 3.21(\mathrm{dd}, J=9.2 \mathrm{~Hz}, 15.2 \mathrm{~Hz}, 1 \mathrm{H}), 2.92$ $(\mathrm{dd}, J=5.6 \mathrm{~Hz}, 14.8 \mathrm{~Hz}, 1 \mathrm{H}), 1.27(\mathrm{t}, J=7.2 \mathrm{~Hz}, 3 \mathrm{H}) ;{ }^{13} \mathrm{C} \mathrm{NMR}\left(100 \mathrm{MHz}, \mathrm{CDCl}_{3}\right) \delta$ 165.9, 163.4 (d, $J=249.7 \mathrm{~Hz}), 128.1$ (d, $J=9.1 \mathrm{~Hz}), 127.8$ (d, $J=3.8 \mathrm{~Hz}), 116.9$ (d, $J=$ $22 \mathrm{~Hz}), 116.0,115.3,64.4,50.9,42.7,38.7,13.6$; HRMS $\left(\mathrm{EI} /\left[\mathrm{M}^{+}\right]\right) \mathrm{m} / \mathrm{z}$ calcd. for $\mathrm{C}_{14} \mathrm{H}_{12} \mathrm{~N}_{2} \mathrm{O}_{2} \mathrm{ClF}$ : 294.0571, found: 294.0578; IR (neat) $v 1734 \mathrm{~cm}^{-1}$.

ent-5f was obtained as a colorless oil in $98 \%$ yield and $95 \%$ ee with 12:1 dr from a reaction catalyzed by QD-2c (10 mol\%) at room temperature for $12 \mathrm{~h}$.

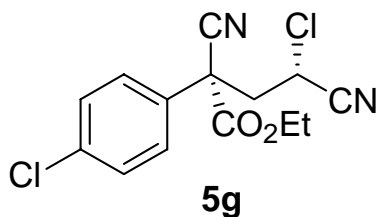

Q-2c (12.0 mg, $10 \mathrm{~mol} \%)$ catalyzed reaction of $\mathbf{3 g}(44.7 \mathrm{mg}, 0.2$ $\mathrm{mmol})$ and $4(52.5 \mathrm{mg}, 0.6 \mathrm{mmol})$ in toluene $(2.0 \mathrm{~mL})$ was run at room temperature for $12 \mathrm{~h}$ to furnish the crude product [ $\mathrm{dr}=$ 13:1, determined by ${ }^{1} \mathrm{H}$ NMR analysis of the crude product]. The crude product was purified by flash chromatography on silica gel (hexanes/ethyl acetate $=$ $6 / 1)$ to give adducts $\mathbf{5} \mathbf{g}$ and $\mathbf{5} \mathbf{g}(\mathrm{dr}=14: 1)$ as a colorless oil in $98 \%$ yield. The ee of the major diastereomer was determined to be $94 \%$ ee [determined by HPLC, Chiralcel OD, 
hexanes: isopropanol $=80: 20,1.0 \mathrm{~mL} / \mathrm{min}, \lambda 220 \mathrm{~nm}, \mathrm{t}($ major $)=21.45 \mathrm{~min}, \mathrm{t}($ minor $)=$ $11.89 \mathrm{~min}] .{ }^{1} \mathrm{H}$ NMR (400 MHz, $\left.\mathrm{CDCl}_{3}\right) \delta 7.50(\mathrm{~d}, J=8.8 \mathrm{~Hz}, 2 \mathrm{H}), 7.44(\mathrm{~d}, J=8.4 \mathrm{~Hz}, 2$ H), $4.61(\mathrm{dd}, J=5.6 \mathrm{~Hz}, 9.2 \mathrm{~Hz}, 1 \mathrm{H}), 4.35-4.20(\mathrm{~m}, 2 \mathrm{H}), 3.22(\mathrm{dd}, J=9.2 \mathrm{~Hz}, 15.2 \mathrm{~Hz}$, $1 \mathrm{H}), 2.90(\mathrm{dd}, J=6.4 \mathrm{~Hz}, 15.6 \mathrm{~Hz}), 1.27(\mathrm{t}, J=7.2 \mathrm{~Hz}, 3 \mathrm{H}) ;{ }^{13} \mathrm{C} \mathrm{NMR}(100 \mathrm{MHz}$, $\left.\mathrm{CDCl}_{3}\right) \delta 165.7,136.3,130.5,129.9,127.4,115.8,115.2,64.5,51.0,42.4,38.7,13.6$; HRMS (ESI/[M+H] $\left.]^{+}\right) \mathrm{m} / \mathrm{z}$ calcd. for $\mathrm{C}_{14} \mathrm{H}_{13} \mathrm{~N}_{2} \mathrm{O}_{2} \mathrm{Cl}_{2}: 311.0354$, found: 311.0365; IR (neat) $v 1747 \mathrm{~cm}^{-1}$.

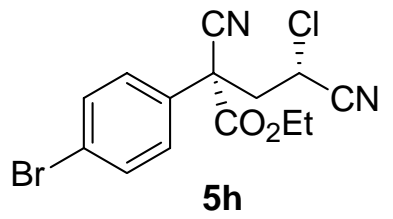

Q-2c (18.0 mg, $10 \mathrm{~mol} \%)$ catalyzed reaction of $\mathbf{3 h}(80.4 \mathrm{mg}, 0.3$ $\mathrm{mmol})$ and $4(78.8 \mathrm{mg}, 0.9 \mathrm{mmol})$ in toluene $(3.0 \mathrm{~mL})$ was run at room temperature for $12 \mathrm{~h}$ to furnish the crude product $[\mathrm{dr}=$ 12:1, determined by ${ }^{1} \mathrm{H}$ NMR analysis of the crude product]. The crude product was purified by flash chromatography on silica gel (hexanes/ethyl acetate $=$ $5 / 1)$ to give adducts $\mathbf{5} \mathbf{h}$ and $\mathbf{5}^{\prime} \mathbf{h}(\mathrm{dr}=14: 1)$ as a colorless oil in $98 \%$ yield. The ee of the major diastereomer was determined to be $95 \%$ ee [determined by HPLC, Chiralcel OD, hexanes: isopropanol $=90: 10,1.0 \mathrm{~mL} / \mathrm{min}, \lambda 220 \mathrm{~nm}, \mathrm{t}($ major $)=63.06 \mathrm{~min}, \mathrm{t}($ minor $)=$ $22.78 \mathrm{~min}] .{ }^{1} \mathrm{H}$ NMR $\left(400 \mathrm{MHz}, \mathrm{CDCl}_{3}\right) \delta 7.60(\mathrm{~d}, J=8.8 \mathrm{~Hz}, 2 \mathrm{H}), 7.43(\mathrm{~d}, J=8.8 \mathrm{~Hz}$, 2H), 4.61 (dd, $J=5.2 \mathrm{~Hz}, 8.8 \mathrm{~Hz}, 1 \mathrm{H}), 4.35-4.20(\mathrm{~m}, 2 \mathrm{H}), 3.22$ (dd, $J=9.2 \mathrm{~Hz}, 14.4 \mathrm{~Hz}$, $1 \mathrm{H}), 2.90(\mathrm{dd}, J=5.6 \mathrm{~Hz}, 15.2 \mathrm{~Hz}, 1 \mathrm{H}), 1.27$ (t, $J=7.2 \mathrm{~Hz}, 3 \mathrm{H}) ;{ }^{13} \mathrm{C}$ NMR $(100 \mathrm{MHz}$, $\left.\mathrm{CDCl}_{3}\right) \delta$ 165.7, 132.9, 131.1, 127.6, 124.4, 115.8, 115.2, 64.5, 51.1, 42.3, 38.7, 13.6; HRMS (EI/[M $\left.{ }^{+}\right]$) m/z calcd. for $\mathrm{C}_{14} \mathrm{H}_{12} \mathrm{~N}_{2} \mathrm{O}_{2} \mathrm{ClBr}$ : 353.9771, found: 353.9762; IR (neat) $v$ $1750 \mathrm{~cm}^{-1}$.

ent-5h was obtained as a colorless oil in $98 \%$ yield and $94 \%$ ee with $12: 1 \mathrm{dr}$ from a reaction catalyzed by QD-2c (10 mol\%) at room temperature for $12 \mathrm{~h}$.

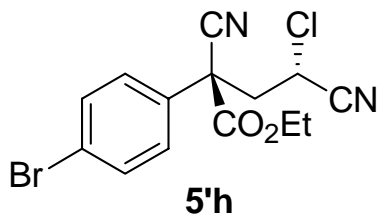

5'h was obtained as a colorless oil in $75 \%$ yield and $80 \%$ ee [determined by HPLC, Chiralcel OD, hexanes: isopropanol $=90$ : $10,1.0 \mathrm{~mL} / \mathrm{min}, \lambda 220 \mathrm{~nm}, \mathrm{t}$ (major) $=16.07 \mathrm{~min}, \mathrm{t}($ minor $)=$ $19.38 \mathrm{~min}$ ] with $3: 1 \mathrm{dr}$ from a reaction catalyzed by Q-1b $(20 \mathrm{~mol} \%)$ at room temperature for $152 \mathrm{~h} .{ }^{1} \mathrm{H}$ NMR $\left(400 \mathrm{MHz}, \mathrm{CDCl}_{3}\right) \delta 7.59(\mathrm{~d}, J=9.2 \mathrm{~Hz}, 2 \mathrm{H}), 7.43(\mathrm{~d}, J=8.8 \mathrm{~Hz}$, 
2H), $4.67(\mathrm{dd}, J=6.4 \mathrm{~Hz}, 8.8 \mathrm{~Hz}, 1 \mathrm{H}), 4.93-4.22(\mathrm{~m}, 2 \mathrm{H}), 3.26(\mathrm{dd}, J=9.2 \mathrm{~Hz}, 12.0 \mathrm{hz}$, $1 \mathrm{H}), 2.76(\mathrm{dd}, J=6.4 \mathrm{~Hz}, 14.8 \mathrm{~Hz}, 1 \mathrm{H}), 1.29$ (t, $J=7.2 \mathrm{~Hz}, 3 \mathrm{H}) ;{ }^{13} \mathrm{C}$ NMR $(100 \mathrm{MHz}$, $\left.\mathrm{CDCl}_{3}\right) \delta 165.4,132.8,131.7,127.5,124.3,115.8,115.1,64.6,51.1,42.9,38.2,13.6$; HRMS (EI/[M $\left.{ }^{+}\right]$) m/z calcd. for $\mathrm{C}_{14} \mathrm{H}_{12} \mathrm{~N}_{2} \mathrm{O}_{2} \mathrm{ClBr}$ : 353.9771, found: 353.9762; IR (neat) $v$ $1747 \mathrm{~cm}^{-1}$.

ent-5'h was obtained as a colorless oil in $98 \%$ yield and $86 \%$ ee with $4: 1 \mathrm{dr}$ from a reaction catalyzed by QD-1b (20 mol\%) at room temperature for $96 \mathrm{~h}$.

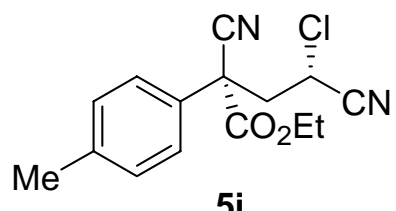

Q-2c (12.0 mg, $10 \mathrm{~mol} \%)$ catalyzed reaction of $3 \mathbf{i}(40.6 \mathrm{mg}, 0.2$ $\mathrm{mmol})$ and $4(52.5 \mathrm{mg}, 0.6 \mathrm{mmol})$ in toluene $(2.0 \mathrm{~mL})$ was run at room temperature for $12 \mathrm{~h}$ to furnish the crude product $[\mathrm{dr}=$ 14:1, determined by ${ }^{1} \mathrm{H}$ NMR analysis of the crude product]. The crude product was purified by flash chromatography on silica gel (hexanes/ethyl acetate $=6 / 1$ ) to give adducts $\mathbf{5 i}$ and $\mathbf{5} \mathbf{i}(\mathrm{dr}=17: 1)$ as a colorless oil in $100 \%$ yield. The ee of the major diastereomer was determined to be $95 \%$ ee [determined by HPLC, Chiralcel OJ, hexanes: isopropanol $=60: 40,1.0 \mathrm{~mL} / \mathrm{min}, \lambda 220 \mathrm{~nm}, \mathrm{t}$ (major) $=58.70 \mathrm{~min}, \mathrm{t}($ minor $)=12.59$ min]. ${ }^{1} \mathrm{H}$ NMR (400 MHz, $\left.\mathrm{CDCl}_{3}\right) \delta 7.41(\mathrm{~d}, J=7.6 \mathrm{~Hz}, 2 \mathrm{H}), 7.25(\mathrm{~d}, J=8.0 \mathrm{~Hz}, 2 \mathrm{H})$, $4.56(\mathrm{dd}, J=5.6 \mathrm{~Hz}, 9.2 \mathrm{~Hz}, 1 \mathrm{H}), 4.34-4.19(\mathrm{~m}, 2 \mathrm{H}), 3.18$ (dd, $J=8.4 \mathrm{~Hz}, 14.8 \mathrm{~Hz}, 1 \mathrm{H})$, 2.94 (dd, $J=5.2 \mathrm{~Hz}, 14.4 \mathrm{~Hz}, 1 \mathrm{H}), 2.38$ (s, 3H), 1.26 (t, $J=7.2 \mathrm{~Hz}, 3 \mathrm{H}) ;{ }^{13} \mathrm{C}$ NMR (100 $\left.\mathrm{MHz}, \mathrm{CDCl}_{3}\right) \delta 166.2,140.1,130.4,128.9,125.7,116.4,115.5,64.1,51.1,42.5,38.8$, 21.0, 13.6; HRMS (ESI/[M+H] $\left.]^{+}\right) \mathrm{m} / \mathrm{z}$ calcd. for $\mathrm{C}_{15} \mathrm{H}_{16} \mathrm{~N}_{2} \mathrm{O}_{2} \mathrm{Cl}$ : 291.0900, found: 291.0911; IR (neat) $v 1747 \mathrm{~cm}^{-1}$.

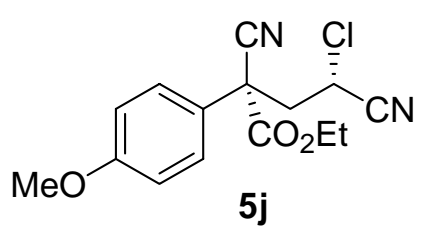

Q-2c (18.0 mg, $10 \mathrm{~mol} \%)$ catalyzed reaction of $\mathbf{3 j}(65.7 \mathrm{mg}$, $0.3 \mathrm{mmol})$ and $4(78.8 \mathrm{mg}, 0.9 \mathrm{mmol})$ in toluene $(3.0 \mathrm{~mL})$ was run at room temperature for $12 \mathrm{~h}$ to furnish the crude product $\left[\mathrm{dr}=13: 1\right.$, determined by ${ }^{1} \mathrm{H}$ NMR analysis of the crude product]. The crude product was purified by flash chromatography on silica gel (hexanes/ethyl acetate $=5 / 1)$ to give adducts $\mathbf{5} \mathbf{j}$ and $\mathbf{5} \mathbf{j}(\mathbf{d r}=20: 1)$ as a colorless oil in $98 \%$ yield. The ee of the major diastereomer was determined to be $95 \%$ ee [determined 
by HPLC, Chiralcel OD, hexanes: isopropanol $=80: 20,1.0 \mathrm{~mL} / \mathrm{min}, \lambda 220 \mathrm{~nm}, \mathrm{t}$ (major) $=20.60 \mathrm{~min}, \mathrm{t}($ minor $)=13.05 \mathrm{~min}] .{ }^{1} \mathrm{H} \mathrm{NMR}\left(400 \mathrm{MHz}, \mathrm{CDCl}_{3}\right) \delta 7.44(\mathrm{~d}, J=8.4 \mathrm{~Hz}$, 2H), $6.95(\mathrm{~d}, J=8.4 \mathrm{~Hz}, 2 \mathrm{H}), 4.57$ (dd, $J=5.6 \mathrm{~Hz}, 8.8 \mathrm{~Hz}, 1 \mathrm{H}), 4.34-4.19(\mathrm{~m}, 2 \mathrm{H}), 3.83$ (s, 3H), 3.16 (dd, $J=9.6 \mathrm{~Hz}, 14.4 \mathrm{~Hz}, 1 \mathrm{H}), 2.94$ (dd, $J=6.0 \mathrm{~Hz}, 15.2 \mathrm{~Hz}, 1 \mathrm{H}), 1.26$ (t, $J$ $=7.2 \mathrm{~Hz}, 3 \mathrm{H}) ;{ }^{13} \mathrm{C} \mathrm{NMR}\left(100 \mathrm{MHz}, \mathrm{CDCl}_{3}\right) \delta 166.2,160.6,127.2,123.6,116.5,115.5$, 115.0, 64.1, 55.4, 50.8, 42.6, 38.8, 13.6; HRMS $\left(\mathrm{EI} /\left[\mathrm{M}^{+}\right]\right) \mathrm{m} / \mathrm{z}$ calcd. for $\mathrm{C}_{15} \mathrm{H}_{15} \mathrm{~N}_{2} \mathrm{O}_{3} \mathrm{Cl}$ : 306.0771, found: 306.0773 ; IR (neat) $v 1748 \mathrm{~cm}^{-1}$.

ent-5j was obtained as a colorless oil in $99 \%$ yield and $95 \%$ ee with $15: 1 \mathrm{dr}$ from a reaction catalyzed by QD-2c (10 mol\%) at room temperature for $12 \mathrm{~h}$.

年 14:1, determined by ${ }^{1} \mathrm{H}$ NMR analysis of the crude product]. The crude product was purified by flash chromatography on silica gel (hexanes/ethyl acetate $=5 / 1$ ) to give adducts $\mathbf{5} \mathbf{k}$ and $\mathbf{5} \mathbf{k}(\mathrm{dr}=17: 1)$ as a colorless oil in $98 \%$ yield. The ee of the major diastereomer was determined to be $95 \%$ ee [determined by HPLC, Chiralcel OD, hexanes: isopropanol $=80: 20,1.0 \mathrm{~mL} / \mathrm{min}, \lambda 254 \mathrm{~nm}, \mathrm{t}($ major $)=90.77 \mathrm{~min}, \mathrm{t}($ minor $)=$ $16.58 \mathrm{~min}] .{ }^{1} \mathrm{H}$ NMR (400 MHz, $\left.\mathrm{CDCl}_{3}\right) \delta 8.10(\mathrm{~s}, 1 \mathrm{H}), 7.95-7.86(\mathrm{~m}, 3 \mathrm{H}), 7.60-7.51(\mathrm{~m}$, $3 \mathrm{H}), 4.58(\mathrm{dd}, J=5.2 \mathrm{~Hz}, 8.8 \mathrm{~Hz}, 1 \mathrm{H}), 4.36-4.19(\mathrm{~m}, 2 \mathrm{H}), 3.30(\mathrm{dd}, J=9.2 \mathrm{~Hz}, 15.6 \mathrm{~Hz}$, $1 \mathrm{H}), 3.06(\mathrm{dd}, J=2.8 \mathrm{~Hz}, 15.2 \mathrm{~Hz}, 1 \mathrm{H}), 1.24(\mathrm{t}, J=7.2 \mathrm{~Hz}, 3 \mathrm{H}) ;{ }^{13} \mathrm{C}$ NMR $(100 \mathrm{MHz}$, $\left.\mathrm{CDCl}_{3}\right) \delta 166.0,133.3,133.0,130.0,129.0,128.4,127.7,127.6,127.4,126.2,122.0$, 116.4, 115.5, 64.3, 51.5, 42.3, 38.9, 13.6; HRMS (EI/[M $\left.\left.\mathrm{M}^{+}\right]\right) \mathrm{m} / \mathrm{z}$ calcd. for $\mathrm{C}_{18} \mathrm{H}_{15} \mathrm{~N}_{2} \mathrm{O}_{2} \mathrm{Cl}$ : 326.0822, found: 326.0815 ; IR (neat) $v 1749 \mathrm{~cm}^{-1}$.

\footnotetext{
CN $\stackrel{C}{\vdots} \quad$ Q-2c $(18.0 \mathrm{mg}, 10 \mathrm{~mol} \%)$ catalyzed reaction of 31 (38.7 mg, $0.3 \mathrm{mmol})$ $\widehat{\overline{C O S M e}}^{\mathrm{CN}}$ 5 and $4(78.8 \mathrm{mg}, 0.9 \mathrm{mmol})$ in toluene $(3.0 \mathrm{~mL})$ was run at room temperature for $12 \mathrm{~h}$ to furnish the crude product [ $\mathrm{dr}=9: 1$, determined by ${ }^{1} \mathrm{H}$ NMR analysis of the crude product]. The crude product was purified by flash
} 
chromatography on silica gel (hexanes/ethyl acetate $=8 / 1)$ to give adducts $\mathbf{5 l}$ and $\mathbf{5} \mathbf{l} \mathbf{~}(\mathrm{dr}$ $=11: 1)$ as a colorless oil in $98 \%$ yield. The ee of the major diastereomer was determined to be $96 \%$ ee [determined by HPLC, Chiralcel OD, hexanes: isopropanol $=90: 10,1.0$ $\mathrm{mL} / \mathrm{min}, \lambda 220 \mathrm{~nm}, \mathrm{t}$ (major) $=22.86 \mathrm{~min}, \mathrm{t}($ minor $)=20.01 \mathrm{~min}] .{ }^{1} \mathrm{H} \mathrm{NMR}(400 \mathrm{MHz}$, $\left.\mathrm{CDCl}_{3}\right) \delta 4.71(\mathrm{dd}, J=6.4 \mathrm{~Hz}, 8.8 \mathrm{~Hz}, 1 \mathrm{H}), 2.89(\mathrm{dd}, J=8.4 \mathrm{~Hz}, 14.4 \mathrm{~Hz}, 1 \mathrm{H}), 2.55(\mathrm{dd}$, $J=6.0 \mathrm{~Hz}, 14.8 \mathrm{~Hz}, 1 \mathrm{H}), 2.44(\mathrm{~s}, 3 \mathrm{H}), 1.73(\mathrm{~s}, 3 \mathrm{H}) ;{ }^{13} \mathrm{C} \mathrm{NMR}\left(100 \mathrm{MHz}, \mathrm{CDCl}_{3}\right) \delta$ 194.8, 117.9, 115.6, 48.5, 42.2, 38.6, 25.2, 12.7; HRMS $\left(\mathrm{EI} /\left[\mathrm{M}^{+}\right]\right) \mathrm{m} / \mathrm{z}$ calcd. for $\mathrm{C}_{8} \mathrm{H}_{9} \mathrm{~N}_{2} \mathrm{OClS}: 216.0124$, found: 216.0120; IR (neat) $v 1684 \mathrm{~cm}^{-1}$.

ent-5l was obtained as a colorless oil in 98\% yield and 96\% ee with 9:1 dr from a reaction catalyzed by QD-2c (10 mol\%) at room temperature for $12 \mathrm{~h}$.

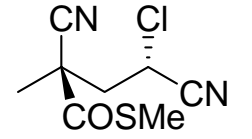

5'।

5'l was obtained as a colorless oil in $44 \%$ yield and $88 \%$ ee with $7: 1 \mathrm{dr}$ from a reaction catalyzed by Q-1b (20 mol\%) at room temperature for 96 h.

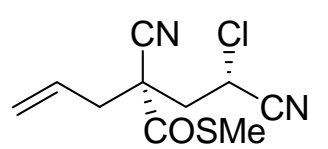

$5 \mathrm{~m}$ Q-2c (18.0 mg, $10 \mathrm{~mol} \%)$ catalyzed reaction of $\mathbf{3 m}(46.5 \mathrm{mg}, 0.3$ $\mathrm{mmol})$ and $4(78.8 \mathrm{mg}, 0.9 \mathrm{mmol})$ in toluene $(3.0 \mathrm{~mL})$ was run at room temperature for $14 \mathrm{~h}$ to furnish the crude product $[\mathrm{dr}=9: 1$, determined by ${ }^{1} \mathrm{H}$ NMR analysis of the crude product]. The crude product was purified by flash chromatography on silica gel (hexanes/ethyl acetate $=6 / 1$ ) to give adduct $\mathbf{5 m}$ and $\mathbf{5}^{\prime} \mathbf{m}(\mathrm{dr}=12: 1)$ as a colorless oil in $99 \%$ yield. The ee of the major diastereomer was determined to be $95 \%$ ee [determined by HPLC, Chiralcel OJ, hexanes: isopropanol $=96$ : $4,1.0 \mathrm{~mL} / \mathrm{min}, \lambda 220 \mathrm{~nm}, \mathrm{t}$ (major) $=65.75 \mathrm{~min}, \mathrm{t}$ (minor) $=80.75 \mathrm{~min}] .{ }^{1} \mathrm{H}$ NMR $(400$ $\left.\mathrm{MHz}, \mathrm{CDCl}_{3}\right) \delta 5.84-5.74(\mathrm{~m}, 1 \mathrm{H}), 5.34(\mathrm{~d}, J=9.6 \mathrm{~Hz}, 1 \mathrm{H}), 5.28(\mathrm{~d}, J=17.2 \mathrm{~Hz}, 1 \mathrm{H})$, $4.70(\mathrm{dd}, J=5.6 \mathrm{~Hz}, 9.2 \mathrm{~Hz}, 1 \mathrm{H}), 2.87(\mathrm{dd}, J=9.2 \mathrm{~Hz}, 14.8 \mathrm{~Hz}, 1 \mathrm{H}), 2.75(\mathrm{dd}, J=6.8$ $\mathrm{Hz}, 14.0 \mathrm{~Hz}, 1 \mathrm{H}), 2.55$ (m, 2H), 2.43 (s, 3H); : ${ }^{13} \mathrm{C}$ NMR (100 MHz, $\left.\mathrm{CDCl}_{3}\right) \delta 194.3$, 128.7, 122.8, 116.7, 115.5, 53.3, 42.6, 40.6, 38.8, 12.8; HRMS (EI/[M $\left.\left.{ }^{+}\right]\right) \mathrm{m} / \mathrm{z}$ calcd. for $\mathrm{C}_{10} \mathrm{H}_{11} \mathrm{~N}_{2} \mathrm{OClS}: 242.0281$, found: 242.0290 ; IR (neat) $v 1684 \mathrm{~cm}^{-1}$. 
ent-5m was obtained as a colorless oil in $98 \%$ yield and $95 \%$ ee with $10: 1 \mathrm{dr}$ from a reaction catalyzed by QD-2c (10 mol\%) at room temperature for $12 \mathrm{~h}$.

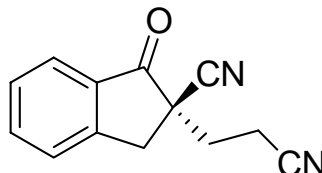

Q-2c (24.0 mg, $20 \mathrm{~mol} \%)$ catalyzed reaction of 3a (31.4 mg, 0.2 $\mathrm{mmol})$ and $6(32.0 \mathrm{mg}, 0.6 \mathrm{mmol})$ in toluene was run at room $(-)-7 a$ temperature for $12 \mathrm{~h}$ to furnish the adduct (-)-7a as a white solid in $100 \%$ yield after flash chromatography (elution gradient: hexanes/ethyl acetate $=2 / 1$ ) and 93\% ee as determined by HPLC analysis [Daicel chiralcel OD, hexanes: isopropanol = $60: 40,1.0 \mathrm{~mL} / \mathrm{min}, \lambda 220 \mathrm{~nm}, \mathrm{t}$ (major) $=21.50 \mathrm{~min}, \mathrm{t}($ minor $)=15.66 \mathrm{~min}] .[\alpha]_{\mathrm{D}}^{25}=$ 45.2 (c 0.92, $\left.\mathrm{CHCl}_{3}\right) ;{ }^{1} \mathrm{H}$ NMR $\left(400 \mathrm{MHz}, \mathrm{CDCl}_{3}\right) \delta 7.84$ (d, $\left.J=8 \mathrm{~Hz}, 1 \mathrm{H}\right), 7.75(\mathrm{t}, J=$ $7.2 \mathrm{~Hz}, 1 \mathrm{H}), 7.52(\mathrm{~m}, 2 \mathrm{H}), 3.76$ (d, $J=17.6 \mathrm{~Hz}, 1 \mathrm{H}), 3.37$ (d, $J=17.6 \mathrm{~Hz}, 1 \mathrm{H}), 2.90-2.82$ (m, $1 \mathrm{H}), 2.78-2.69(\mathrm{~m}, 1 \mathrm{H}), 2.38-2.31(\mathrm{~m}, 1 \mathrm{H}), 2.24-2.17(\mathrm{~m}, 1 \mathrm{H}) ;{ }^{13} \mathrm{C}$ NMR $(100 \mathrm{MHz}$, $\left.\mathrm{CDCl}_{3}\right) \delta 196.3,150.2,137.0,132.4,129.1,126.6,125.8,118.0,117.9,46.5,38.4,31.9$, 13.6; HRMS (EI/[M+]) m/z calcd. for $\mathrm{C}_{13} \mathrm{H}_{10} \mathrm{~N}_{2} \mathrm{O}: 210.0793$, found: 210.0789 ; IR (neat) $v$ $1734 \mathrm{~cm}^{-1}$.

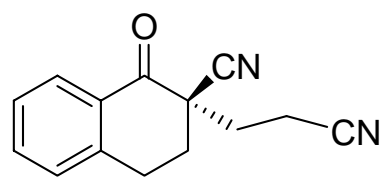

$(+)-7 b$

Q-2c (24.0 mg, $20 \mathrm{~mol} \%)$ catalyzed reaction of $\mathbf{3 b}(34.2 \mathrm{mg}, 0.2$ $\mathrm{mmol})$ and $6(32.0 \mathrm{mg}, 0.6 \mathrm{mmol})$ in toluene was run at room temperature for $36 \mathrm{~h}$ to furnish the adduct $(+)-7 \mathbf{b}$ as a colorless oil in $92 \%$ yield after flash chromatography (elution gradient: hexanes/ethyl acetate=4/1) and 94\% ee as determined by HPLC analysis [Daicel chiralcel OD, hexanes: isopropanol $=80: 20,1.0 \mathrm{~mL} / \mathrm{min}, \lambda 220 \mathrm{~nm}, \mathrm{t}$ (major) $=30.63 \mathrm{~min}, \mathrm{t}$ $($ minor $)=23.87 \mathrm{~min}] .[\alpha]_{\mathrm{D}}^{25}=+35.6\left(c \quad 0.96, \mathrm{CHCl}_{3}\right) ;{ }^{1} \mathrm{H}$ NMR $\left(400 \mathrm{MHz}, \mathrm{CDCl}_{3}\right) \delta$ $8.03(\mathrm{~d}, J=7.6 \mathrm{~Hz}, 1 \mathrm{H}), 7.59$ (t, $J=7.6 \mathrm{~Hz}, 1 \mathrm{H}), 7.39$ (t, $J=7.6 \mathrm{~Hz}, 1 \mathrm{H}), 7.30(\mathrm{~d}, J=8.0$ $\mathrm{Hz}, 1 \mathrm{H}), 3.39-3.32(\mathrm{~m}, 1 \mathrm{H}), 3.15-3.08(\mathrm{~m}, 1 \mathrm{H}), 2.84-2.76(\mathrm{~m}, 1 \mathrm{H}), 2.73-2.65(\mathrm{~m}, 1 \mathrm{H})$, 2.61-2.47 (m, 2H), 2.33-2.26 (m, 1H), 2.23-2.16 (m, 1H); ${ }^{13} \mathrm{C}$ NMR (100 MHz, $\left.\mathrm{CDCl}_{3}\right)$ $\delta 189.2,142.3,135.1,129.4,129.0,128.9,127.6,118.3,117.2,46.9,32.3,29.9,25.5$, 13.7; HRMS (EI/[M $\left.\left.{ }^{+}\right]\right) \mathrm{m} / \mathrm{z}$ calcd. for $\mathrm{C}_{14} \mathrm{H}_{12} \mathrm{~N}_{2} \mathrm{O}$ : 224.0950, found: 224.0947; IR (neat) $v$ $1700 \mathrm{~cm}^{-1}$. 


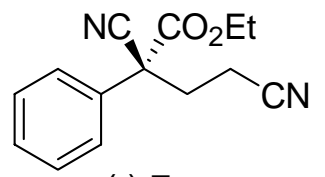

$(-)-7 e$

Q-2c $(24.0 \mathrm{mg}, 20 \mathrm{~mol} \%)$ catalyzed reaction of $3 \mathbf{e}(38.0 \mathrm{mg}, 0.2$ $\mathrm{mmol})$ and $6(32.0 \mathrm{mg}, 0.6 \mathrm{mmol})$ in toluene was run at room temperature for $96 \mathrm{~h}$ to furnish the adduct (-)-7e as a colorless oil in 95\% yield after flash chromatography (elution gradient: hexanes/ethyl acetate $=5 / 1$ ) and 89\% ee as determined by HPLC analysis [Daicel chiralcel OD, hexanes: isopropanol $=$ $80: 20,1.0 \mathrm{~mL} / \mathrm{min}, \lambda 220 \mathrm{~nm}, \mathrm{t}$ (major) $=17.64 \mathrm{~min}, \mathrm{t}($ minor $)=14.04 \mathrm{~min}] .[\alpha]_{\mathrm{D}}^{25}=$ 23.6 (c 1.16, $\left.\mathrm{CHCl}_{3}\right) ;{ }^{1} \mathrm{H}$ NMR (400 MHz, $\left.\mathrm{CDCl}_{3}\right) \delta$ 7.52-7.43 (m, 5H), $4.26(\mathrm{~m}, 2 \mathrm{H})$, $2.76(\mathrm{~m}, 1 \mathrm{H}), 2.55(\mathrm{~m}, 2 \mathrm{H}), 2.39(\mathrm{~m}, 1 \mathrm{H}), 1.25$ (t, $J=7.2 \mathrm{~Hz}, 3 \mathrm{H}) ;{ }^{13} \mathrm{C} \mathrm{NMR}(100 \mathrm{MHz}$, $\left.\mathrm{CDCl}_{3}\right) \delta 166.2,132.5,129.6,125.7,117.5,116.8,63.9,52.8,33.6,13.8,13.7$; HRMS $\left(\mathrm{EI} /\left[\mathrm{M}^{+}\right]\right) \mathrm{m} / \mathrm{z}$ calcd. for $\mathrm{C}_{14} \mathrm{H}_{14} \mathrm{~N}_{2} \mathrm{O}_{2}: 242.1055$, found: 242.1063; IR (neat) $\vee 1734 \mathrm{~cm}^{-1}$.

$(+)-7$ e was obtained as a colorless oil in $93 \%$ yield and $90 \%$ ee from a reaction catalyzed by QD-2c (20 mol\%) at room temperature for $96 \mathrm{~h}$.

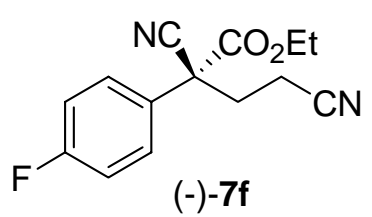

Q-2c (24.0 mg, $20 \mathrm{~mol} \%)$ catalyzed reaction of $\mathbf{3 f}(41.4 \mathrm{mg}, 0.2$ $\mathrm{mmol})$ and $6(32.0 \mathrm{mg}, 0.6 \mathrm{mmol})$ in toluene was run at room temperature for $120 \mathrm{~h}$ to furnish the adduct (-)-7f as a colorless oil in $84 \%$ yield after flash chromatography (elution gradient: hexanes/ethyl acetate $=5 / 1$ ) and $88 \%$ ee as determined by HPLC analysis [Daicel chiralpak AD, hexanes: isopropanol $=$ 98:2, $1.0 \mathrm{~mL} / \mathrm{min}, \lambda 220 \mathrm{~nm}, \mathrm{t}$ (major) $=22.86 \mathrm{~min}, \mathrm{t}$ (minor) $=20.00 \mathrm{~min}] .[\alpha]_{\mathrm{D}}{ }^{25}=-$ $22.4\left(\right.$ c 1.09, $\left.\mathrm{CHCl}_{3}\right) ;{ }^{1} \mathrm{H}$ NMR (400 MHz, $\left.\mathrm{CDCl}_{3}\right) \delta 7.51(\mathrm{dd}, J=4.8 \mathrm{~Hz}, 9.2 \mathrm{~Hz}, 2 \mathrm{H})$, $7.16(\mathrm{t}, J=8.0 \mathrm{~Hz}, 2 \mathrm{H}), 4.34-4.21(\mathrm{~m}, 2 \mathrm{H}), 2.80-2.71(\mathrm{~m}, 1 \mathrm{H}), 2.60-2.39(\mathrm{~m}, 3 \mathrm{H}), 1.26(\mathrm{t}$, $J=7.2 \mathrm{~Hz}, 3 \mathrm{H}) ;{ }^{13} \mathrm{C}$ NMR $\left(100 \mathrm{MHz}, \mathrm{CDCl}_{3}\right) \delta 166.1,163.2(\mathrm{~d}, J=248.9 \mathrm{~Hz}), 128.4(\mathrm{~d}$, $J=3.8 \mathrm{~Hz}), 127.8(\mathrm{~d}, J=8.4 \mathrm{~Hz}), 117.3,116.7(\mathrm{~d}, J=22.1 \mathrm{~Hz}), 116.7,64.0,52.3,33.7$, 13.8, 13.7; HRMS (EI/[M+]) m/z calcd. for $\mathrm{C}_{14} \mathrm{H}_{13} \mathrm{~N}_{2} \mathrm{O}_{2} \mathrm{~F}: 260.0961$, found: 260.0966; IR (neat) $v 1747 \mathrm{~cm}^{-1}$.

$(+)-7 \mathbf{f}$ was obtained as a colorless oil in $87 \%$ yield and $90 \%$ ee from a reaction catalyzed by QD-2c (20 mol\%) at room temperature for $72 \mathrm{~h}$. 


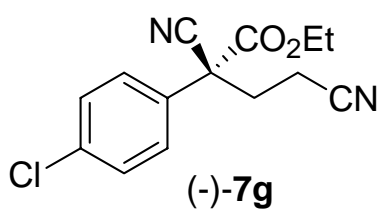

Q-2c (24.0 mg, $20 \mathrm{~mol} \%)$ catalyzed reaction of $\mathbf{3 g}(44.7 \mathrm{mg}, 0.2$ $\mathrm{mmol})$ and $6(32.0 \mathrm{mg}, 0.6 \mathrm{mmol})$ in toluene was run at room temperature for $52 \mathrm{~h}$ to furnish the adduct (-)-7 $\mathrm{g}$ as a colorless oil in $96 \%$ yield after flash chromatography (elution gradient: hexanes/ethyl acetate $=5 / 1$ ) and $88 \%$ ee as determined by HPLC analysis [Daicel chiralcel OJ, hexanes: isopropanol $=$ $60: 40,0.8 \mathrm{~mL} / \mathrm{min}, \lambda 220 \mathrm{~nm}, \mathrm{t}$ (major) $=38.01 \mathrm{~min}, \mathrm{t}($ minor $)=28.57 \mathrm{~min}] .[\alpha]_{\mathrm{D}}{ }^{25}=-$ 15.0 (c 1.07, $\left.\mathrm{CHCl}_{3}\right) ;{ }^{1} \mathrm{H}$ NMR (400 MHz, $\left.\mathrm{CDCl}_{3}\right) \delta 7.44(\mathrm{~m}, 4 \mathrm{H}), 4.32-4.22(\mathrm{~m}, 2 \mathrm{H})$, 2.79-2.71 (m, 1H), 2.60-2.38 (m, 3H), $1.26(\mathrm{t}, J=7.2 \mathrm{~Hz}, 3 \mathrm{H}) ;{ }^{13} \mathrm{C}$ NMR $(100 \mathrm{MHz}$, $\left.\mathrm{CDCl}_{3}\right) \delta 165.9,135.9,131.1,129.8,127.2,117.3,116.5,64.1,52.4,33.5,13.8,13.7$; HRMS (EI/[M+ $\left.\left.{ }^{+}\right]\right) \mathrm{m} / \mathrm{z}$ calcd. for $\mathrm{C}_{14} \mathrm{H}_{13} \mathrm{~N}_{2} \mathrm{O}_{2} \mathrm{Cl}$ : 276.0666, found: 276.0671; IR (neat) $v$ $1733 \mathrm{~cm}^{-1}$.

$(+)-7 \mathrm{~g}$ was obtained as a colorless oil in $96 \%$ yield and $89 \%$ ee from a reaction catalyzed by QD-2c (20 mol\%) at room temperature for $52 \mathrm{~h}$.

Q-2c $(24.0 \mathrm{mg}, 20 \mathrm{~mol} \%)$ catalyzed reaction of $\mathbf{3 h}(53.6 \mathrm{mg}, 0.2$
mmol) and $\mathbf{6}(32.0 \mathrm{mg}, 0.6 \mathrm{mmol})$ in toluene was run at room in $89 \%$ yield after flash chromatography (elution gradient: hexanes/ethyl acetate=5/1) and $89 \%$ ee as determined by HPLC analysis $[(\mathrm{R}, \mathrm{R})-$ Whelk-O 1 , hexanes: isopropanol $=$ 97:3, $1.0 \mathrm{~mL} / \mathrm{min}, \lambda 220 \mathrm{~nm}, \mathrm{t}$ (major) $=18.39 \mathrm{~min}, \mathrm{t}($ minor $)=22.44 \mathrm{~min}] .[\alpha]_{\mathrm{D}}{ }^{25}=$ 11.2 (c 0.98, $\mathrm{CHCl}_{3}$ ); ${ }^{1} \mathrm{H} \mathrm{NMR}\left(400 \mathrm{MHz}, \mathrm{CDCl}_{3}\right) \delta 7.59$ (d, $\left.J=8.8 \mathrm{~Hz}, 2 \mathrm{H}\right), 7.39$ (d, $J=$ $8.8 \mathrm{~Hz}, 2 \mathrm{H}), 4.34-4.20(\mathrm{~m}, 2 \mathrm{H}), 2.79-2.71(\mathrm{~m}, 1 \mathrm{H}), 2.61-2.38(\mathrm{~m}, 3 \mathrm{H}), 1.26(\mathrm{t}, J=7.2$ $\mathrm{Hz}, 3 \mathrm{H}) ;{ }^{13} \mathrm{C} \mathrm{NMR}\left(100 \mathrm{MHz}, \mathrm{CDCl}_{3}\right) \delta 165.8,132.8,131.7,127.5,124.1,117.3,116.4$, 64.2, 52.5, 33.5, 13.8, 13.7; HRMS (EI/[M $\left.\left.{ }^{+}\right]\right) \mathrm{m} / \mathrm{z}$ calcd. for $\mathrm{C}_{14} \mathrm{H}_{13} \mathrm{~N}_{2} \mathrm{O}_{2} \mathrm{Br}$ : 320.0160, found: 320.0167 ; IR (neat) $v 1747 \mathrm{~cm}^{-1}$.

$(+)-7 \mathrm{~h}$ was obtained as a colorless oil in $82 \%$ yield and $89 \%$ ee from a reaction catalyzed by QD-2c (20 mol\%) at room temperature for $72 \mathrm{~h}$. 


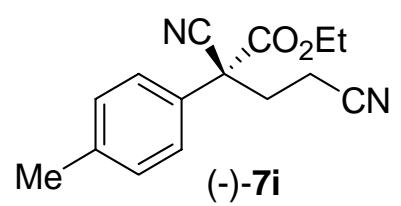

Q-2c $(24.0 \mathrm{mg}, 20 \mathrm{~mol} \%)$ catalyzed reaction of $3 \mathbf{i}(40.6 \mathrm{mg}, 0.2$ $\mathrm{mmol})$ and $6(32.0 \mathrm{mg}, 0.6 \mathrm{mmol})$ in toluene was run at room temperature for $114 \mathrm{~h}$ to furnish the adduct (-)-7i as a colorless oil in 92\% yield after flash chromatography (elution gradient: hexanes/ethyl acetate $=5 / 1$ ) and $90 \%$ ee as determined by HPLC analysis [Daicel chiralcel OJ, hexanes: isopropanol $=60: 40,1.0 \mathrm{~mL} / \mathrm{min}, \lambda 220 \mathrm{~nm}, \mathrm{t}$ (major) $=30.98 \mathrm{~min}, \mathrm{t}($ minor $)=21.24 \mathrm{~min}] .[\alpha]_{\mathrm{D}}{ }^{25}=-$ 19.5 (c 0.91, $\left.\mathrm{CHCl}_{3}\right) ;{ }^{1} \mathrm{H}$ NMR $\left(400 \mathrm{MHz}, \mathrm{CDCl}_{3}\right) \delta 7.37$ (d, $\left.J=8.0 \mathrm{~Hz}, 2 \mathrm{H}\right), 7.24$ (d, $J=$ $8.8 \mathrm{~Hz}, 2 \mathrm{H}), 4.31-4.20(\mathrm{~m} 2 \mathrm{H}), 2.76-2.70(\mathrm{~m}, 1 \mathrm{H}), 2.55-2.47$ (m, 2H), 2.41-2.35 (m, 1H), $2.37(\mathrm{~s}, 3 \mathrm{H}), 1.25(\mathrm{t}, J=7.2 \mathrm{~Hz}, 3 \mathrm{H}) ;{ }^{13} \mathrm{C} \mathrm{NMR}\left(100 \mathrm{MHz}, \mathrm{CDCl}_{3}\right) \delta 166.3,139.7,130.2$, 129.5, 125.6, 117.6, 117.0, 63.8, 52.5, 33.6, 21.0, 13.70, 13.68; HRMS (EI/[M $\left.\left.{ }^{+}\right]\right) \mathrm{m} / \mathrm{z}$ calcd. for $\mathrm{C}_{15} \mathrm{H}_{16} \mathrm{~N}_{2} \mathrm{O}_{2}: 256.1212$, found: 256.1227 ; IR (neat) $v 1749 \mathrm{~cm}^{-1}$.

Q-2c $(24.0 \mathrm{mg}, 20 \mathrm{~mol} \%)$ catalyzed reaction of $\mathbf{3 j}(44.0 \mathrm{mg}$,
$0.2 \mathrm{mmol})$ and $\mathbf{6}(32.0 \mathrm{mg}, 0.6 \mathrm{mmol})$ in toluene was run at
room temperature for $117 \mathrm{~h}$ to furnish the adduct (-)-7j as a colorless oil in $85 \%$ yield after flash chromatography (elution gradient: hexanes/ethyl acetate $=5 / 1$ ) and $90 \%$ ee as determined by HPLC analysis [Daicel chiralcel OD, hexanes: isopropanol $=80: 20,1.0 \mathrm{~mL} / \mathrm{min}, \lambda 220 \mathrm{~nm}, \mathrm{t}($ major $)=17.34 \mathrm{~min}, \mathrm{t}($ minor $)=23.57$ $\min ] .[\alpha]_{D}{ }^{25}=-18.3\left(c \quad 0.98, \mathrm{CHCl}_{3}\right) ;{ }^{1} \mathrm{H}$ NMR $\left(400 \mathrm{MHz}, \mathrm{CDCl}_{3}\right) \delta 7.41(\mathrm{~d}, J=8.4 \mathrm{~Hz}$, $2 \mathrm{H}), 6.94(\mathrm{~d}, J=8.4 \mathrm{~Hz}, 2 \mathrm{H}), 4.28-4.23(\mathrm{~m}, 2 \mathrm{H}), 3.83(\mathrm{~s}, 3 \mathrm{H}), 2.72(\mathrm{~m}, 1 \mathrm{H}), 2.55-2.50$ (m, 2H), 2.42-2.37 (m, 1H), $1.25(\mathrm{t}, J=7.2 \mathrm{~Hz}, 3 \mathrm{H}) ;{ }^{13} \mathrm{C} \mathrm{NMR}\left(100 \mathrm{MHz}, \mathrm{CDCl}_{3}\right) \delta$ 166.4, 160.3, 127.1, 124.2, 117.6, 117.1, 114.8, 63.8, 55.4, 52.2, 33.6, 13.7; HRMS $\left(\mathrm{EI} /\left[\mathrm{M}^{+}\right]\right) \mathrm{m} / \mathrm{z}$ calcd. for $\mathrm{C}_{15} \mathrm{H}_{16} \mathrm{~N}_{2} \mathrm{O}_{3}: 272.1161$, found: 272.1163; IR (neat) $v 1734 \mathrm{~cm}^{-1}$.

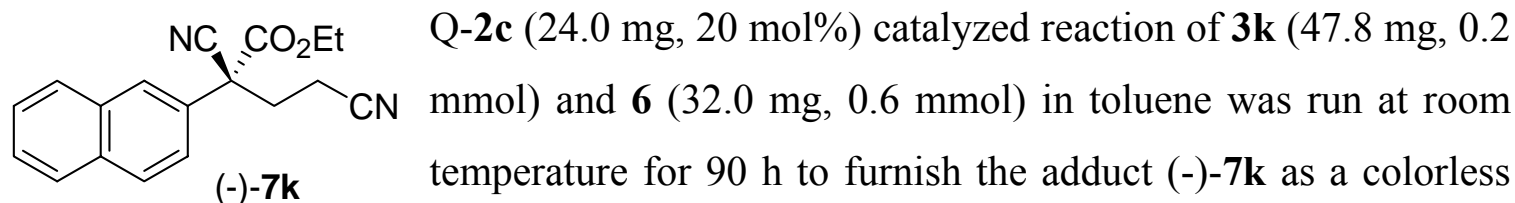
oil in $89 \%$ yield after flash chromatography (elution gradient: hexanes/ethyl acetate $=5 / 1$ ) and $89 \%$ ee as determined by HPLC analysis [ $(\mathrm{R}, \mathrm{R})$-Whelk-O 1 , hexanes: isopropanol $=$ $80: 20,1.0 \mathrm{~mL} / \mathrm{min}, \lambda 280 \mathrm{~nm}, \mathrm{t}$ (major) $=9.57 \mathrm{~min}, \mathrm{t}$ (minor) $=12.38 \mathrm{~min}] .[\alpha]_{\mathrm{D}}{ }^{25}=$ - 
27.0 (c 1.07, $\left.\mathrm{CHCl}_{3}\right) ;{ }^{1} \mathrm{H}$ NMR (400 MHz, $\left.\mathrm{CDCl}_{3}\right) \delta 8.04(\mathrm{~s}, 1 \mathrm{H}), 7.93-7.86(\mathrm{~m}, 3 \mathrm{H})$, 7.59-7.49 (m, 3H), 4.33-4.21 (m, 2H), 2.89-2.82 (m, 1H), 2.68-2.54 (m, 2H), 2.42-2.35 $(\mathrm{m}, 1 \mathrm{H}), 1.24(\mathrm{t}, J=7.2 \mathrm{~Hz}, 3 \mathrm{H}) ;{ }^{13} \mathrm{C}$ NMR $\left(100 \mathrm{MHz}, \mathrm{CDCl}_{3}\right) \delta 166.2,133.2,133.0$, $129.9,129.6,128.3,127.7,127.5,127.3,126.0,122.0,117.6,116.9,64.0,53.0,33.5$, 13.8, 13.7; HRMS (EI/[M+]) m/z calcd. for $\mathrm{C}_{18} \mathrm{H}_{16} \mathrm{~N}_{2} \mathrm{O}_{2}: 292.1212$, found: 292.1208; IR (neat) $v 1749 \mathrm{~cm}^{-1}$.

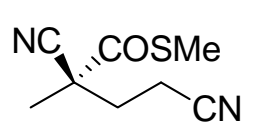

$(-)-71$

Q-2c $(24.0 \mathrm{mg}, 20 \mathrm{~mol} \%)$ catalyzed reaction of $\mathbf{3 1}(25.8 \mathrm{mg}, 0.2 \mathrm{mmol})$ and $6(32.0 \mathrm{mg}, 0.6 \mathrm{mmol})$ in toluene was run at room temperature for 72 $\mathrm{h}$ to furnish the adduct (-)-7l as a colorless oil in $80 \%$ yield after flash chromatography (elution gradient: hexanes/ethyl acetate=4/1) and 93\% ee as determined by HPLC analysis [Daicel chiralpak AS, hexanes: isopropanol $=80: 20,1.0 \mathrm{~mL} / \mathrm{min}, \lambda$ $220 \mathrm{~nm}, \mathrm{t}($ major $)=19.79 \mathrm{~min}, \mathrm{t}($ minor $)=24.11 \mathrm{~min}] .[\alpha]_{\mathrm{D}}{ }^{25}=-5.1\left(\right.$ c $\left.1.07, \mathrm{CHCl}_{3}\right) ;{ }^{1} \mathrm{H}$ NMR (400 MHz, $\left.\mathrm{CDCl}_{3}\right)$ \& 2.64-2.39 (m, 3H), 2.43 (s, 3H), 2.19-2.11 (m, 1H), 1.68 (s, $3 \mathrm{H}) ;{ }^{13} \mathrm{C} \mathrm{NMR}\left(100 \mathrm{MHz}, \mathrm{CDCl}_{3}\right) \delta 195.1,118.6,117.5,49.8,33.5,24.2,13.7,12.6$; HRMS (ESI/[M+H] $]^{+}$m/z calcd. for $\mathrm{C}_{8} \mathrm{H}_{11} \mathrm{~N}_{2} \mathrm{OS}: 183.0592$, found: 183.0597; IR (neat) $v$ $1684 \mathrm{~cm}^{-1}$.

$\underbrace{\mathrm{NCN}}_{(-)-7 \mathrm{~m}} \mathrm{CO}$ mmol) and $6(32.0 \mathrm{mg}, 0.6 \mathrm{mmol})$ in toluene was run at room temperature for $88 \mathrm{~h}$ to furnish the adduct (-)-7m as a colorless oil in $82 \%$ yield after flash chromatography (elution gradient: hexanes/ethyl acetate $=5 / 1$ ) and $91 \%$ ee as determined by HPLC analysis [Daicel chiralpak AS, hexanes: isopropanol $=85: 15,1.0$ $\mathrm{mL} / \mathrm{min}, \lambda 220 \mathrm{~nm}, \mathrm{t}$ (major) $=21.38 \mathrm{~min}, \mathrm{t}$ (minor) $=18.55 \mathrm{~min}] .[\alpha]_{\mathrm{D}}{ }^{25}=-35.5$ (c 0.93 , $\left.\mathrm{CHCl}_{3}\right) ;{ }^{1} \mathrm{H} \mathrm{NMR}\left(400 \mathrm{MHz}, \mathrm{CDCl}_{3}\right) \delta 5.81-5.72(\mathrm{~m}, 1 \mathrm{H}), 5.30(\mathrm{~d}, J=10.4 \mathrm{~Hz}, 1 \mathrm{H}), 5.26$ $(\mathrm{d}, J=16.8 \mathrm{~Hz}, 1 \mathrm{H}), 2.73(\mathrm{~m}, 1 \mathrm{H}), 2.64-2.34(\mathrm{~m}, 4 \mathrm{H}), 2.43(\mathrm{~s}, 3 \mathrm{H}), 2.20-2.12(\mathrm{~m}, 1 \mathrm{H})$; ${ }^{13} \mathrm{C}$ NMR $\left(100 \mathrm{MHz}, \mathrm{CDCl}_{3}\right) \delta 194.4,129.1,122.2,117.6,117.5,54.9,41.8,32.0,13.6$, 12.7; HRMS (EI/[M $\left.{ }^{+}\right]$) m/z calcd. for $\mathrm{C}_{10} \mathrm{H}_{12} \mathrm{~N}_{2} \mathrm{OS}: 208.0670$, found: 208.0680; IR (neat) $\checkmark 1684 \mathrm{~cm}^{-1}$. 


\section{Absolute configuration determination of compounds 5a, 5l, 7a, 71 .}

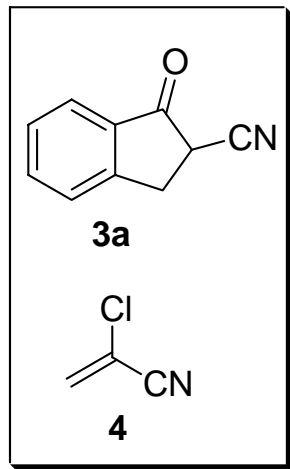

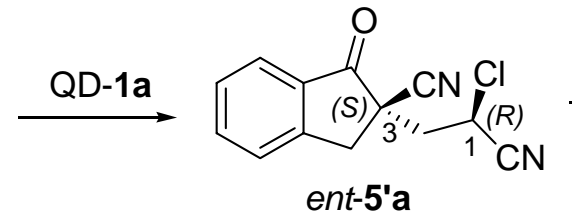

$\underset{\mathrm{Et}_{2} \mathrm{O}, \mathrm{rt}}{\stackrel{\mathrm{Zn} / \mathrm{AcOH}}{\longrightarrow}}$

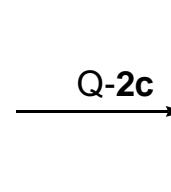

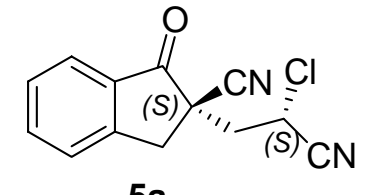

$5 a$

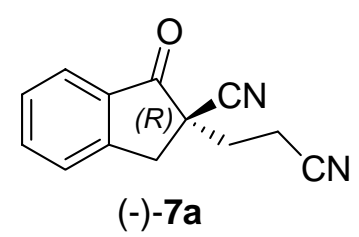

$(-)-7 a$<smiles>N#CCC[C@]1(C#N)Cc2ccccc2C1=O</smiles>

$(-)-7 a$
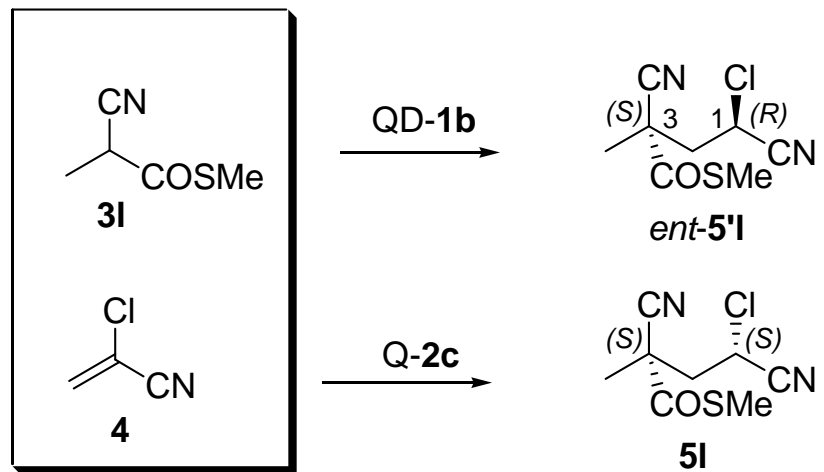

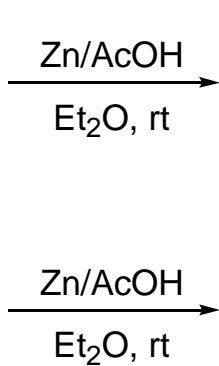

$\mathrm{Et}_{2} \mathrm{O}, \mathrm{rt}$
(S)<smiles>COC(=O)CC1CCCC1</smiles>

$(-)-71$

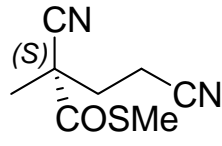

$(-)-71$

As determined previously by us, ${ }^{11}$ compound ent-5'a (obtained from QD-1a catalyzed reaction of $\mathbf{3 a}$ and $\mathbf{4})$ has the absolute configuration of $(1 R, 3 S)$ as shown above. Compound ent-5'l (obtained from QD-1b catalyzed reaction of $\mathbf{3 1}$ and 4) has the absolute configuration of $(1 R, 3 S)$ as shown above. ${ }^{11}$

To a solution of ent-5'a $(24.5 \mathrm{mg}, 0.1 \mathrm{mmol})$ in diethyl ether $(0.5 \mathrm{~mL})$ was added acetic acid $(0.5 \mathrm{~mL})$ and zinc powder $(26.2 \mathrm{mg}, 0.4 \mathrm{mmol})$. The resulting mixture was stirred at room temperature for $20 \mathrm{~h}$. TLC analysis showed ent-5'a was completely consumed. The reaction mixture was diluted with diethyl ether $(5 \mathrm{~mL})$, washed with water $(5 \mathrm{~mL} \times 3)$ and brine, dried over anhydrous $\mathrm{Na}_{2} \mathrm{SO}_{4}$. Purification by flash chromatography on silica gel (hexanes/ethyl acetate $=2 / 1$ ) afforded dechlorination product (-)-7a in quantitative yield. Thus (-)-7a has the absolute configuration of $R$. According to the same procedure, the dechlorination of 5a (obtained from Q-2c catalyzed reaction of $\mathbf{3 a}$ and 4) was performed. The dechlorination product was found to be (-)-7a as shown by comparison with the 
dechlorination product obtained from ent-5'a via HPLC analysis, thus 5a was determined to have the absolute configuration of $(1 S, 3 S)$. The respective dechlorination of ent-5'l and $5 \mathbf{l}$ afforded the same product (-)-7l by the same procedures, thus (-)-7l has the absolute configuration of $S$, and $\mathbf{5 l}$ has the absolute configuration of $(1 S, 3 S)$. 


\section{Formal synthesis of Manzacidin C. ${ }^{12,13}$}
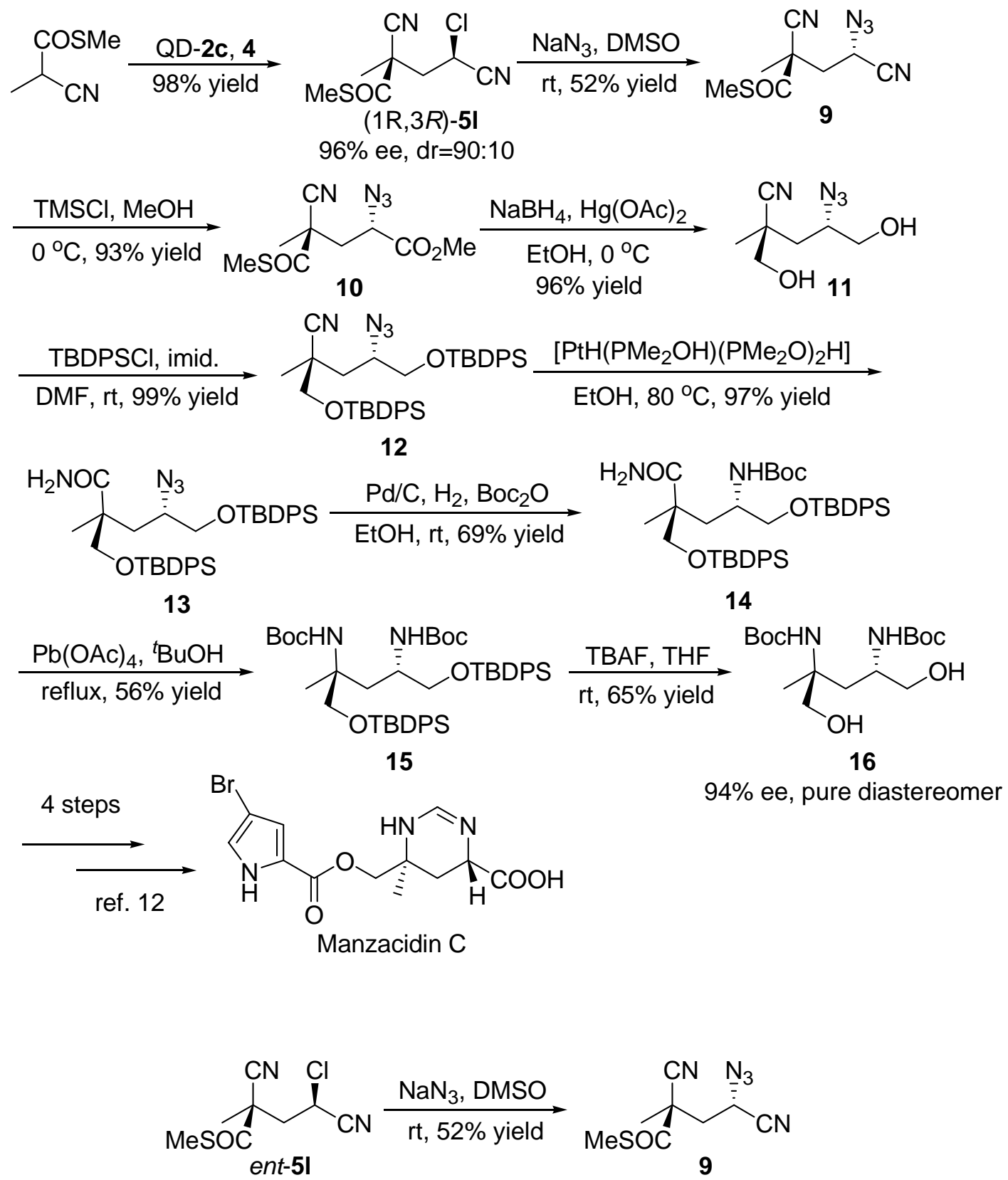

To a solution of $(1 R, 3 R)-5 \mathrm{l}(1.07 \mathrm{~g}, 4.94 \mathrm{mmol}, \mathrm{dr}=90: 10)$ in DMSO (25 mL) was added $\mathrm{NaN}_{3}$ (482 mg, $7.41 \mathrm{mmol}$ ) at room temperature. After stirring at the same temperature for $42 \mathrm{~h}$, the reaction mixture was diluted with water $(50 \mathrm{~mL})$, and extracted with diethyl ether $(2 \times 50 \mathrm{~mL})$. The combined organic layers were washed with water $(4 \times 50 \mathrm{~mL})$ and brine, dried over anhydrous $\mathrm{Na}_{2} \mathrm{SO}_{4}$. After removal of the solvent under reduced 
pressure, the residue was subjected to flash chromatography on silica gel (hexanes /ethyl acetate $=5 / 1$ ) to afford 9 as a colorless oil (573 mg, 52\% yield) in 89:11 dr. ${ }^{1} \mathrm{H}$ NMR (400 $\left.\mathrm{MHz}, \mathrm{CDCl}_{3}\right) \delta 4.42(\mathrm{dd}, J=5.6 \mathrm{~Hz}, 8.0 \mathrm{~Hz}, 1 \mathrm{H}), 2.52(\mathrm{dd}, J=6.0 \mathrm{~Hz}, 14.8 \mathrm{~Hz}, 1 \mathrm{H})$, 2.41 (s, 3H), 2.24 (dd, $J=8.4 \mathrm{~Hz}, 14.4 \mathrm{~Hz}, 1 \mathrm{H}), 1.69$ (s, 3H); ${ }^{13} \mathrm{C} \mathrm{NMR}(100 \mathrm{MHz}$, $\left.\mathrm{CDCl}_{3}\right) \delta 195.0,118.3,114.8,47.9,47.7,39.6,25.4,12.7 ;$ HRMS $\left(\mathrm{CI} /[\mathrm{M}+\mathrm{H}]^{+}\right) \mathrm{m} / \mathrm{z}$ calcd. for $\mathrm{C}_{8} \mathrm{H}_{10} \mathrm{ON}_{5} \mathrm{~S}: 224.0606$, found: 224.0605; IR (neat) $v 1682,2117 \mathrm{~cm}^{-1}$.

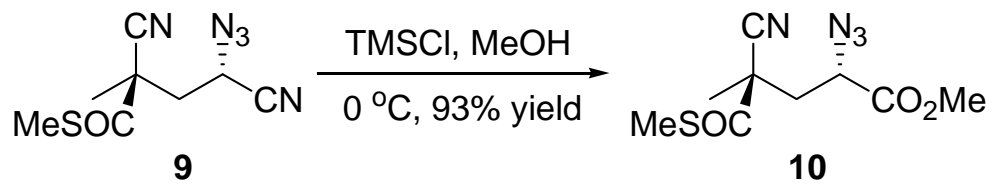

A solution of $9(705 \mathrm{mg}, 3.16 \mathrm{mmol})$ and $\mathrm{TMSCl}(1.45 \mathrm{~mL})$ in anhydrous $\mathrm{MeOH}(6.6$ $\mathrm{mL}$ ) was kept at $0{ }^{\circ} \mathrm{C}$ without stirring. After $48 \mathrm{~h}$, the reaction mixture was diluted with water $(30 \mathrm{~mL})$, extracted with ethyl acetate $(3 \times 30 \mathrm{~mL})$. The combined organic layers were washed with brine, dried over anhydrous $\mathrm{Na}_{2} \mathrm{SO}_{4}$. After removal of the solvent under reduced pressure, the residue was subjected to flash chromatography on silica gel (hexanes /ethyl acetate $=5 / 1$ ) to afford $\mathbf{1 0}$ as a colorless oil (753 $\mathrm{mg}, 93 \%$ yield) in 83:17 dr. ${ }^{1} \mathrm{H}$ NMR (400 MHz, $\left.\mathrm{CDCl}_{3}\right) \delta 4.07$ (dd, $\left.J=4.0 \mathrm{~Hz}, 9.6 \mathrm{~Hz}, 1 \mathrm{H}\right), 3.83(\mathrm{~s}, 3 \mathrm{H}), 2.55$ (dd, $4.0 \mathrm{~Hz}, 14.4 \mathrm{~Hz}, 1 \mathrm{H}$ ), 2.42 (s, 3H), 2.15 (dd, $J=9.2 \mathrm{~Hz}, 14.8 \mathrm{~Hz}, 1 \mathrm{H}$ ), 1.71 (s, 3H); ${ }^{13} \mathrm{C}$ NMR $\left(100 \mathrm{MHz}, \mathrm{CDCl}_{3}\right) \delta 195.8,169.3,119.0,58.7,53.0,48.2,38.2,25.3,12.5$; HRMS $\left(\mathrm{CI} /[\mathrm{M}+\mathrm{H}]^{+}\right) \mathrm{m} / \mathrm{z}$ calcd. for $\mathrm{C}_{9} \mathrm{H}_{13} \mathrm{O}_{3} \mathrm{~N}_{4} \mathrm{~S}: 257.0708$, found: 257.0711 ; IR (neat) $v$ $1682,1747,2114 \mathrm{~cm}^{-1}$.

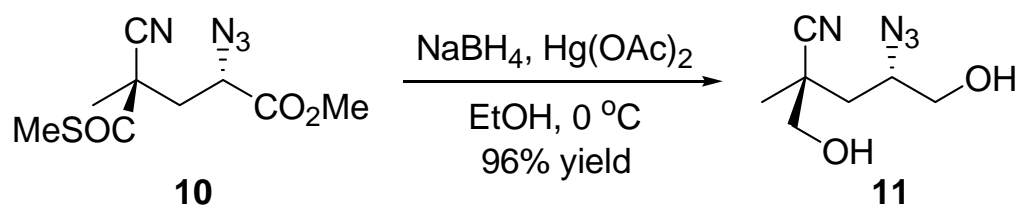

To a solution of $10(666 \mathrm{mg}, 2.60 \mathrm{mmol})$ in EtOH was added $\mathrm{Hg}(\mathrm{OAc})_{2}(828.5 \mathrm{mg}, 2.60$ mmol) at $0{ }^{\circ} \mathrm{C}$. The resulting mixture was stirred for $10 \mathrm{mins}$, and $\mathrm{NaBH}_{4}(786 \mathrm{mg}, 20.8$ 
mmol) was added in portions. After 20 min, the reaction was quenched with saturated aq. $\mathrm{NH}_{4} \mathrm{Cl}$ solution, and the resulting mixture was extracted with ethyl acetate $(4 \times 50 \mathrm{~mL})$. The combined organic layers were washed brine, dried over anhydrous $\mathrm{Na}_{2} \mathrm{SO}_{4}$. After removal of the solvent under reduced pressure, the residue was subjected to flash chromatography on silica gel (hexanes /ethyl acetate=1/1) to afford $\mathbf{1 1}$ as a colorless oil (461 mg, 96\% yield) in 86:14 dr. ${ }^{1} \mathrm{H}$ NMR $\left(400 \mathrm{MHz}, \mathrm{CD}_{3} \mathrm{OD}\right) \delta 3.72-3.51(\mathrm{~m}, 5 \mathrm{H}), 1.82$ (dd, $J=2.4 \mathrm{~Hz}, 14.4 \mathrm{~Hz}, 1 \mathrm{H}), 1.49$ (dd, $J=9.6 \mathrm{~Hz}, 14.8 \mathrm{~Hz}, 1 \mathrm{H}), 1.38(\mathrm{~s}, 3 \mathrm{H}) ;{ }^{13} \mathrm{C} \mathrm{NMR}$ $\left(100 \mathrm{MHz}, \mathrm{CD}_{3} \mathrm{OD}\right) \delta 125.5,69.8,67.9,64.0,40.9,38.5,23.7 ; \mathrm{HRMS}\left(\mathrm{CI} /[\mathrm{M}+\mathrm{H}]^{+}\right) \mathrm{m} / \mathrm{z}$ calcd. for $\mathrm{C}_{7} \mathrm{H}_{13} \mathrm{O}_{2} \mathrm{~N}_{4}$ : 185.1039 , found: 185.1040 ; IR (neat) $v 2115,3418 \mathrm{~cm}^{-1}$.

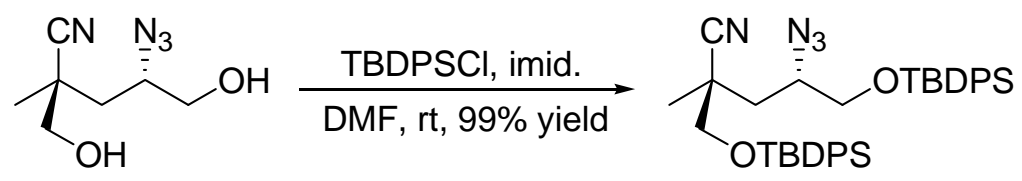

11

12

To a solution of 11 (396 mg, $2.15 \mathrm{mmol})$ and imidazole (442 $\mathrm{mg}, 6.46 \mathrm{mmol})$ in anhydrous DMF $(3.0 \mathrm{~mL})$ was added tert-butyl chlorodiphenylsilane $(1.22 \mathrm{~mL}, 4.73$ mmol) at room temperature. After stirring at the same temperature for $12 \mathrm{~h}$, the reaction was quenched with water $(30 \mathrm{~mL})$. The reaction mixture was extracted with diethyl ether $(3 \times 30 \mathrm{~mL})$. The combined organic layers were washed with water $(3 \times 30 \mathrm{~mL})$ and brine, dried over anhydrous $\mathrm{Na}_{2} \mathrm{SO}_{4}$. After removal of the solvent under reduced pressure, the residue was subjected to flash chromatography on silica gel (hexanes /ethyl acetate=20/1) to afford 12 as a colorless oil (1.41 g, 99\% yield) in 86:14 dr. ${ }^{1} \mathrm{H}$ NMR (400 MHz, $\left.\mathrm{CDCl}_{3}\right) \delta$ 7.68-7.63 (m, 8H), 7.46-7.38 (m, 12H), 3.74-3.52 (m, 5H), $1.81(\mathrm{~d}, J=14.8 \mathrm{~Hz}$, $1 \mathrm{H}), 1.54-1.48(\mathrm{~m}, 1 \mathrm{H}), 1.41(\mathrm{~s}, 3 \mathrm{H}), 1.07(\mathrm{~s}, 18 \mathrm{H}) ;{ }^{13} \mathrm{C} \mathrm{NMR}\left(100 \mathrm{MHz}, \mathrm{CDCl}_{3}\right) \delta$ $139.9,135.61,135.57,135.54,132.6,132.4,132.3,130.04,130.01,129.9,127.88$, 127.86, 122.5, 68.9, 67.6, 60.6, 38.5, 35.9, 26.71, 26.66, 21.5, 19.3, 19.1; HRMS $\left(\mathrm{ESI} /[\mathrm{M}+\mathrm{H}]^{+}\right) \mathrm{m} / \mathrm{z}$ calcd. for $\mathrm{C}_{39} \mathrm{H}_{49} \mathrm{O}_{2} \mathrm{~N}_{4} \mathrm{Si}_{2}:$ 661.3394, found: 661.3371; IR (neat) $v$ $2116 \mathrm{~cm}^{-1}$. 


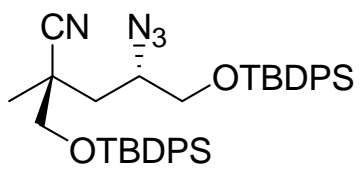

12

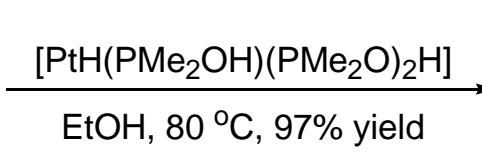

$\mathrm{EtOH}, 80^{\circ} \mathrm{C}, 97 \%$ yield

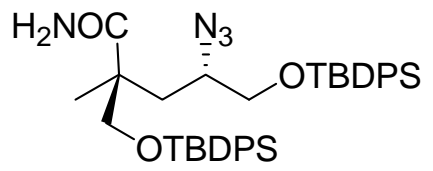

13

To a solution of 12 (465 $\mathrm{mg}, 0.7 \mathrm{mmol})$ in $\mathrm{EtOH}(2.0 \mathrm{~mL})$ was added $\left[\mathrm{PtH}\left(\mathrm{PMe}_{2} \mathrm{OH}\right)\left(\mathrm{PMe}_{2} \mathrm{O}\right)_{2} \mathrm{H}\right](15.7 \mathrm{mg}, 0.035 \mathrm{mmol})$. The resulting mixture was stirred at $80{ }^{\circ} \mathrm{C}$ for $12 \mathrm{~h}$. After cooled to room temperature, the reaction mixture was diluted with water $(20 \mathrm{~mL})$, and extracted with EtOAc $(3 \times 20 \mathrm{~mL})$. The combined organic layers were washed with brine, dried over anhydrous $\mathrm{Na}_{2} \mathrm{SO}_{4}$. After removal of the solvent under reduced pressure, the residue was subjected to flash chromatography on silica gel (hexanes /ethyl acetate $=4 / 1$ ) to afford 13 as a colorless oil (460 mg, 97\% yield) in 86:14 dr. ${ }^{1} \mathrm{H}$ NMR (400 MHz, $\left.\mathrm{CDCl}_{3}\right) \delta$ 7.68-7.63 (m, 8H), 7.43-7.35 (m, 12H), 6.57 (brs, 1H), 6.00 (brs, 1H), 3.71-3.53 (m, 5H), 1.78 (d, $J=14.0 \mathrm{~Hz}, 1 \mathrm{H}), 1.39$ (dd, $J=9.2 \mathrm{~Hz}, 14.4$ $\mathrm{Hz}, 1 \mathrm{H}), 1.23(\mathrm{~s}, 3 \mathrm{H}), 1.07(\mathrm{~s}, 18 \mathrm{H}) ;{ }^{13} \mathrm{C} \mathrm{NMR}\left(100 \mathrm{MHz}, \mathrm{CDCl}_{3}\right) \delta 178.2,135.53$, 135.50, 132.80, 132.76, 132.4, 132.3, 129.9, 129.7, 127.8, 127.7, 69.3, 68.0, 60.4, 46.3, 35.7, 26.8, 26.6, 19.6, 19.2, 19.0; HRMS (ESI/[M+H] $]^{+}$) m/z calcd. for $\mathrm{C}_{39} \mathrm{H}_{51} \mathrm{O}_{3} \mathrm{~N}_{4} \mathrm{Si}_{2}$ : 679.3500, found: 679.3472 ; IR (neat) $v 1676 \mathrm{~cm}^{-1}$.

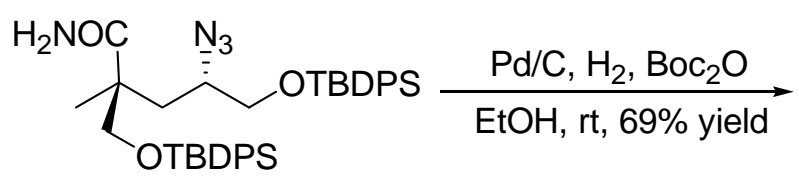

13

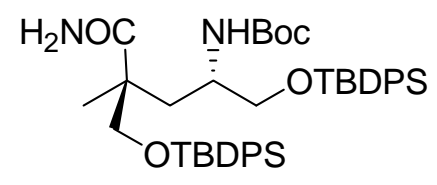

14

To a suspension of $\mathbf{1 3}(1.172 \mathrm{~g}, 1.73 \mathrm{mmol}), 10 \%$ palladium on carbon (150 $\mathrm{mg})$ in EtOH $(20 \mathrm{~mL})$ was added $\mathrm{Boc}_{2} \mathrm{O}(755 \mathrm{mg}, 3.46 \mathrm{mmol})$. The resulting mixture was stirred under $\mathrm{H}_{2}$ atmosphere (1 atm) for 23 at room temperature, followed by filtration on Cellite ${ }^{\circledR} 521$. The filtrate was concentrated in vacuo, and then subjected to flash chromatography on silica gel (hexanes /ethyl acetate $=5 / 1)$ to afford 14 as a colorless oil ( $900 \mathrm{mg}, 69 \%$ yield). The dr of 14 can not be determined by ${ }^{1} \mathrm{H}$ NMR. ${ }^{1} \mathrm{H}$ NMR (400 MHz, $\left.\mathrm{CDCl}_{3}\right) \delta$ 7.66-7.60 
(m, 8H), 7.44-7.34 (m, 12H), 6.88 (brs, 1H), 5.50 (brs, 1H), 4.73 (d, J=9.2 Hz, 1H), 3.98 (d, $J=10.4 \mathrm{~Hz}, 1 \mathrm{H}), 3.73(\mathrm{~m}, 1 \mathrm{H}), 3.51(\mathrm{~m}, 3 \mathrm{H}), 2.00-1.93(\mathrm{~m}, 1 \mathrm{H}), 1.60(\mathrm{~d}, J=12.4 \mathrm{~Hz}$, 1H), 1.41 (s, 9H), 1.21 (s, 3H), 1.08 (s, 9H), $1.03(\mathrm{~s}, 9 \mathrm{H}) ;{ }^{13} \mathrm{C} \mathrm{NMR}\left(100 \mathrm{MHz}, \mathrm{CDCl}_{3}\right) \delta$ 179.2, 155.2, 135.6, 135.5, 133.3, 133.2, 132.5, 129.9, 129.7, 129.6, 127.8, 127.8, 127.7, 127.6, 78.8, 48.6, 45.8, 37.5, 28.4, 28.2, 26.9, 26.8, 21.9, 19.3, 19.2; HRMS $\left(\mathrm{ESI} /[\mathrm{M}+\mathrm{H}]^{+}\right) \mathrm{m} / \mathrm{z}$ calcd. for $\mathrm{C}_{44} \mathrm{H}_{61} \mathrm{O}_{5} \mathrm{~N}_{2} \mathrm{Si}_{2}:$ 753.4119, found: 753.4099; IR (neat) $v$ $1678,1710 \mathrm{~cm}^{-1}$.

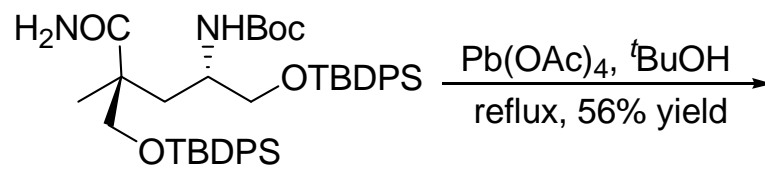

14

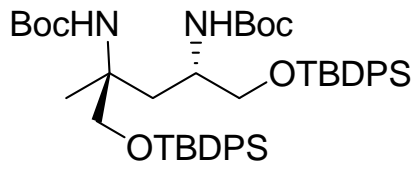

15

To a solution of $14(247 \mathrm{mg}, 0.33 \mathrm{mmol})$ in ${ }^{t} \mathrm{BuOH}(8.0 \mathrm{~mL})$ was added $\mathrm{Pb}(\mathrm{OAc})_{4}(291$ $\mathrm{mg}, 0.66 \mathrm{mmol}$ ) at room temperature. The resulting suspension was heated to reflux for 3 h. After cooled to room temperature, the reaction mixture was diluted with diethyl ether $(30 \mathrm{~mL})$, and quenched with saturated aq. $\mathrm{NaHCO}_{3}$ solution $(20 \mathrm{~mL})$. The aqueous phase was extracted with diethyl ether $(3 \times 20 \mathrm{~mL})$. The combined organic layers were washed with brine and dried over anhydrous $\mathrm{Na}_{2} \mathrm{SO}_{4}$. After removal of the solvent under reduced pressure, the residue was subjected to flash chromatography on silica gel (hexanes /ethyl acetate $=15 / 1)$ to afford $\mathbf{1 5}$ as a white amorphous solid (152 mg, 56\% yield) in 80:20 dr. ${ }^{1} \mathrm{H}$ NMR (400 MHz, $\mathrm{CDCl}_{3}$ ) $\delta 7.64$ (m, 8H), 7.42-7.35 (m, 10H), 4.96 (brs, 1H), 4.67 (d, $J=8.4 \mathrm{~Hz}, 1 \mathrm{H}), 3.82(\mathrm{~m}, 1 \mathrm{H}), 3.73-3.57(\mathrm{~m}, 4 \mathrm{H}), 2.08(\mathrm{~d}, J=14.0 \mathrm{~Hz}, 1 \mathrm{H}), 1.76-1.65$ (m, 1H), 1.39 (s, 9H), 1.38 (s, 9H), 1.29 (s, 3H), 1.07 (s, 9H), 1.06 (s, 9H); ${ }^{13} \mathrm{C} \mathrm{NMR}$ $\left(100 \mathrm{MHz}, \mathrm{CDCl}_{3}\right) \delta 155.1,154.8,135.6,133.32,133.26,133.20,129.7,127.7,78.9$, 68.4, 66.9, 55.6, 48.5, 37.5, 28.4, 26.92, 26.88, 19.4, 19.3; HRMS (ESI/[M+H] $\left.{ }^{+}\right) \mathrm{m} / \mathrm{z}$ calcd. for $\mathrm{C}_{48} \mathrm{H}_{69} \mathrm{O}_{6} \mathrm{~N}_{2} \mathrm{Si}_{2}: 825.4694$, found: 825.4672; IR (neat) $v 1714 \mathrm{~cm}^{-1}$. 


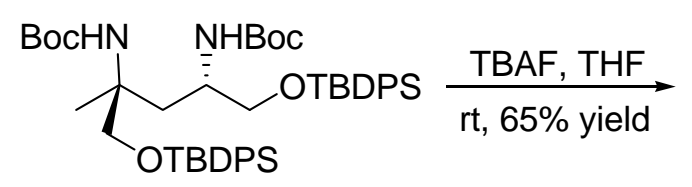

15

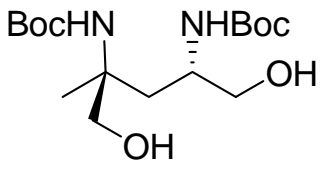

16

To a solution of $15(254 \mathrm{mg}, 0.31 \mathrm{mmol}, \mathrm{dr}=80: 20)$ in THF was added TBAF (330 mg, $1.26 \mathrm{mmol}$ ) at room temperature. After stirring for $1 \mathrm{~h}$, the reaction was quenched with saturated aq. $\mathrm{NH}_{4} \mathrm{Cl}$ solution. The resulting mixture was diluted with brine $(30 \mathrm{~mL})$, and extracted with EtOAc $(3 \times 30 \mathrm{~mL})$. The combined organic layers were washed with brine and dried over anhydrous $\mathrm{Na}_{2} \mathrm{SO}_{4}$. After removal of the solvent under reduced pressure, the residue was subjected to flash chromatography on silica gel (hexanes /ethyl acetate $=1 / 1)$ to afford 16 in diastereomerically pure form as a colorless oil $(70 \mathrm{mg}$, $65 \%$ yield). The ee of 16 was determined by transforming the diol to diester $17 .[\alpha]_{\mathrm{D}}{ }^{25}=$ $+1.8\left(\right.$ c 1.07, $\left.\mathrm{CHCl}_{3}\right) ;{ }^{1} \mathrm{H} \mathrm{NMR}\left(400 \mathrm{MHz}, \mathrm{CDCl}_{3}\right) \delta 5.41$ (s, 1H), 5.16 (s, 1H), 4.64 (brs, 1H), 3.70-3.62 (m 1H), 3.60-3.50 (m, 4H), 3.29 (brs, 1H), 1.98 (dd, J=3.6 Hz, $14.8 \mathrm{~Hz}$, $1 \mathrm{H}), 1.81(\mathrm{dd}, J=7.6 \mathrm{~Hz}, 14.4 \mathrm{~Hz}, 1 \mathrm{H}), 1.40$ (s, 9H), 1.39 (s, 9H), 1.19 (s, 3H); ${ }^{13} \mathrm{C}$ NMR $\left(100 \mathrm{MHz}, \mathrm{CDCl}_{3}\right) \delta 156.53,156.24,79.87,79.71,69.19,66.40,55.99,49.14$, 36.68, 28.37, 28.32, 22.73; HRMS (ESI/[M+H] $]^{+}$) m/z calcd. for $\mathrm{C}_{16} \mathrm{H}_{33} \mathrm{O}_{6} \mathrm{~N}_{2}: 349.2339$, found: 349.2337; IR (neat) $\vee 1694,3352 \mathrm{~cm}^{-1}$. The analytic data of 16 was consistent with the reported data of the intermediate for the synthesis of manzacidin $\mathrm{C}^{12}$ (S-Table 2).

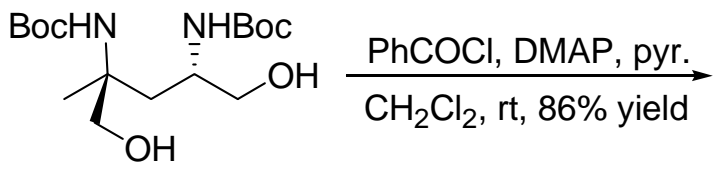

16

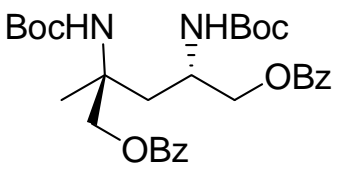

17

To a solution of $16(20 \mathrm{mg}, 0.057 \mathrm{mmol})$ in $\mathrm{CH}_{2} \mathrm{Cl}_{2}(0.5 \mathrm{~mL})$ was added pyridine $(23 \mathrm{mg}$, $0.29 \mathrm{mmol})$, DMAP (2.0 mg), and benzoyl chloride (32 $\mathrm{mg}, 0.23 \mathrm{mmol}$ ) sequentially. The resulting solution was stirred at room temperature for $1 \mathrm{~h}$. The reaction mixture was diluted with EtOAc $(20 \mathrm{~mL})$, washed with water $(10 \mathrm{~mL})$ and brine, dried over anhydrous 
$\mathrm{Na}_{2} \mathrm{SO}_{4}$. After removal of the solvent under reduced pressure, the residue was subjected to flash chromatography on silica gel (hexanes /ethyl acetate $=5 / 1$ ) to afford $\mathbf{1 7}$ as a white amorphous solid (27.6 $\mathrm{mg}, 86 \%$ yield). The ee of $\mathbf{1 7}$ was determined to be $94 \%$ ee [determined by HPLC, Chiralcel OD, hexanes: isopropanol $=97: 3,1.0 \mathrm{~mL} / \mathrm{min}, \lambda 220$ $\mathrm{nm}, \mathrm{t}($ major $)=16.79 \mathrm{~min}, \mathrm{t}($ minor $)=13.68 \mathrm{~min}] .[\alpha]_{\mathrm{D}}{ }^{25}=-1.1\left(\right.$ c $\left.1.10, \mathrm{CHCl}_{3}\right) ;{ }^{1} \mathrm{H}$ NMR (400 MHz, $\left.\mathrm{CDCl}_{3}\right) \delta 8.03(\mathrm{~m}, 4 \mathrm{H}), 7.58-7.54$ (m, 2H), 7.47-7.40 (m, 4H), 4.90 (s, 1H), 4.78 (d, $J=8.0 \mathrm{~Hz}, 1 \mathrm{H}), 4.54$ (d, $J=10.8 \mathrm{~Hz}, 1 \mathrm{H}), 4.37$ (d, $J=11.2 \mathrm{~Hz}, 1 \mathrm{H}), 4.29$ (m, 3H), 2.34 (d, $J=14.0 \mathrm{~Hz}, 1 \mathrm{H}), 1.88$ (dd, $J=8.0 \mathrm{~Hz}, 14.8 \mathrm{~Hz}, 1 \mathrm{H}), 1.43$ (s, 3H), 1.41 (s, 9H), 1.39 (s, 9H); ${ }^{13} \mathrm{C}$ NMR (100 MHz, $\left.\mathrm{CDCl}_{3}\right) \delta 166.4,166.1,155.2,154.5,133.13$, $133.08,129.69,129.61,128.42,128.33,79.7,68.5,67.7,54.4,46.0,37.2,28.32,28.27$, 22.8; HRMS (ESI/[M+H] $]^{+}$) m/z calcd. for $\mathrm{C}_{30} \mathrm{H}_{41} \mathrm{O}_{8} \mathrm{~N}_{2}$ : 557.2863, found: 557.2851; IR (neat) $v 1714 \mathrm{~cm}^{-1}$. 
S-Table 2. Comparison of characteristic data of 16 with that of literature reported ${ }^{12}$.

\begin{tabular}{|c|c|c|}
\hline & 16 & $\begin{array}{c}\text { Manzacidin } \mathrm{C} \\
\text { intermediate, ref.12 }\end{array}$ \\
\hline Optical rotation & $\begin{array}{l}{[\alpha]_{\mathrm{D}}^{25}=+1.8} \\
\left(c 1.07, \mathrm{CHCl}_{3}\right)\end{array}$ & $\begin{array}{l}{[\alpha]_{\mathrm{D}}^{20}=+3.9} \\
\left(c 1.30, \mathrm{CHCl}_{3}\right)\end{array}$ \\
\hline $\begin{array}{l}{ }^{1} \mathrm{H} \text { NMR } \\
\left(400 \mathrm{MHz}, \mathrm{CDCl}_{3}\right)\end{array}$ & $\begin{array}{l}5.41(\mathrm{~s}, 1 \mathrm{H}) \\
5.16(\mathrm{~s}, 1 \mathrm{H}) \\
4.64(\mathrm{brs}, 1 \mathrm{H}) \\
3.70-3.62(\mathrm{~m}, 1 \mathrm{H}) \\
3.60-3.50(\mathrm{~m}, 4 \mathrm{H}) \\
3.29(\mathrm{brs}, 1 \mathrm{H}) \\
1.98(\mathrm{dd}, \mathrm{J}=3.6 \mathrm{~Hz}, 14.8 \mathrm{~Hz}, 1 \mathrm{H}) \\
1.81(\mathrm{dd}, \mathrm{J}=7.6 \mathrm{~Hz}, 14.4 \mathrm{~Hz}, 1 \mathrm{H}) \\
1.40(\mathrm{~s}, 9 \mathrm{H}) \\
1.39(\mathrm{~s}, 9 \mathrm{H}) \\
1.19(\mathrm{~s}, 3 \mathrm{H})\end{array}$ & $\begin{array}{l}5.40(\mathrm{~s}, 1 \mathrm{H}) \\
5.23(\mathrm{~s}, 1 \mathrm{H}) \\
\\
3.66(\mathrm{~m}, 1 \mathrm{H}) \\
3.60-3.50(\mathrm{~m}, 5 \mathrm{H}) \\
\\
1.98(\mathrm{dd}, \mathrm{J}=3.8 \mathrm{~Hz}, 14.5 \mathrm{~Hz}, 1 \mathrm{H}) \\
1.81(\mathrm{dd}, \mathrm{J}=7.8 \mathrm{~Hz}, 14.5 \mathrm{~Hz}, 1 \mathrm{H}) \\
1.41(\mathrm{~s}, 9 \mathrm{H}) \\
1.35(\mathrm{~s}, 9 \mathrm{H}) \\
1.20(\mathrm{~s}, 3 \mathrm{H})\end{array}$ \\
\hline $\begin{array}{l}{ }^{13} \mathrm{C} \mathrm{NMR} \\
\left(100 \mathrm{MHz}, \mathrm{CDCl}_{3}\right)\end{array}$ & $\begin{array}{l}156.53 \\
156.24 \\
79.87 \\
79.71 \\
69.19 \\
66.40 \\
55.99 \\
49.14 \\
36.68 \\
28.37 \\
28.32 \\
22.73\end{array}$ & $\begin{array}{l}156.43 \\
156.18 \\
79.78 \\
79.69 \\
69.05 \\
66.21 \\
55.93 \\
49.11 \\
36.80 \\
28.37 \\
28.33 \\
22.65\end{array}$ \\
\hline
\end{tabular}




\section{References:}

1) Johnson, W. S.; Anderson, J. M.; Shelerg, W. E. J. Am. Chem. Soc. 1944, 66, 218.

2) Johnson, W. S.; Sherleg. W. E. J. Am. Chem. Soc. 1945, 67, 1745

3) Hirota, T.; Ieno, K.; Sasaki, K. J. Heterocyclic Chem. 1986, 23, 1685.

4) Liu, H.-J.; Ly, T.-W.; Tai, C.-L.; Wu, J.-D.; Liang, J.-K.; Guo, J.-C.; Tseng, N.-W.; Shia, K.-S. Tetrahedron 2003, 59, 1209.

5) (a) Kaiser, E. M.; Solter, L. E.; Schwarz, R. A.; Beard, R. D.; Hause, C. R. J. Am. Chem. Soc. 1971, 93, 4237; (b) Albarella, J. M. J. Org. Chem. 1977, 42, 2009.

6) Yokoyama, M.; Hatanaka, H.; Sasaki, A.; Shiraishi, T.; Kumata, K.; Sakamoto, K.; Ogata, K. J. Chem. Soc., Perkin 1 1986, 1197.

7) (a) Samii, Z. K. M. A. E.; Ashmawy, M. I. A.; Mellor, J. M. J. Chem. Soc., Perkin 1 1988, 2523. (b) Jevric, M.; Taylor, D. K.; Greatrex, B. W.; Tiekink, E. R. T. Tetrahedron 2005, 61, 1885. (c) Heaney, F.; Fenlon, J.; McArdle, P.; Cunningham, D. Org. Biomol. Chem. 2003, 1, 1122.

8) Hiratake, J.; Inagaki, M.; Yamamoto, Y.; Oda, J. J. Chem. Soc., Perkin 1 1987, 1053.

9) (a) Li, H.; Wang, Y.; Tang, L.; Deng, L. J. Am. Chem. Soc. 2004, 126, 9906. (b) Li, H.; Wang, Y.; Tang, L.; Wu, F.; Liu, X.; Guo, C.; Foxman, B. M.; Deng, L. Angew. Chem. Int. Ed. 2005, 44, 105.

10) (a) Vakulya, B.; Varga, S.; Csámpai, A.; Soós, T. Org Lett. 2005, 7, 1967. (b) Li, B.; Jiang, L.; Liu, M.; Chen, Y.; Ding, L.; Wu, Y. Synlett 2005, 4, 603.

11) Wang, Y.; Liu, X.; Deng, L. J. Am. Chem. Soc. 2006, 128, 3928.

12) Namba, K.; Shinada, T.; Teramoto, T.; Ohfune, Y. J. Am. Chem. Soc. 2000, 122, 10708.

13) For other asymmetric total syntheses of manzacidins, see: (a) Wehn, P. M.; Du Bois, J. J. Am. Chem. Soc. 2002, 124, 12950. (b) Lanter, J. C.; Chen, H.; Zhang, X.; Sui, Z. Org. Lett. 2005, 7, 5905. (c) Kano, T.; Hashimoto, T.; Maruoka, K. J. Am. Chem. Soc. 2006, 128, 2174. 
HPLC conditions for ee determination: Chiralpak $\mathrm{AD} \times 2$, hexanes: isopropanol $=80: 20$, $1.0 \mathrm{~mL} / \mathrm{min}, 220 \mathrm{~nm}$.

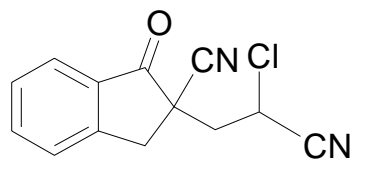

Racemic-5a<smiles>N#CC(Cl)C[C@]1(C#N)Cc2ccccc2C1=O</smiles>

$5 a$

97\% ee, product obtained with Q-2c

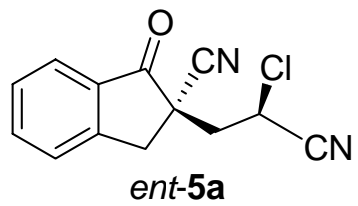

97\% ee, product obtained with QD-2c
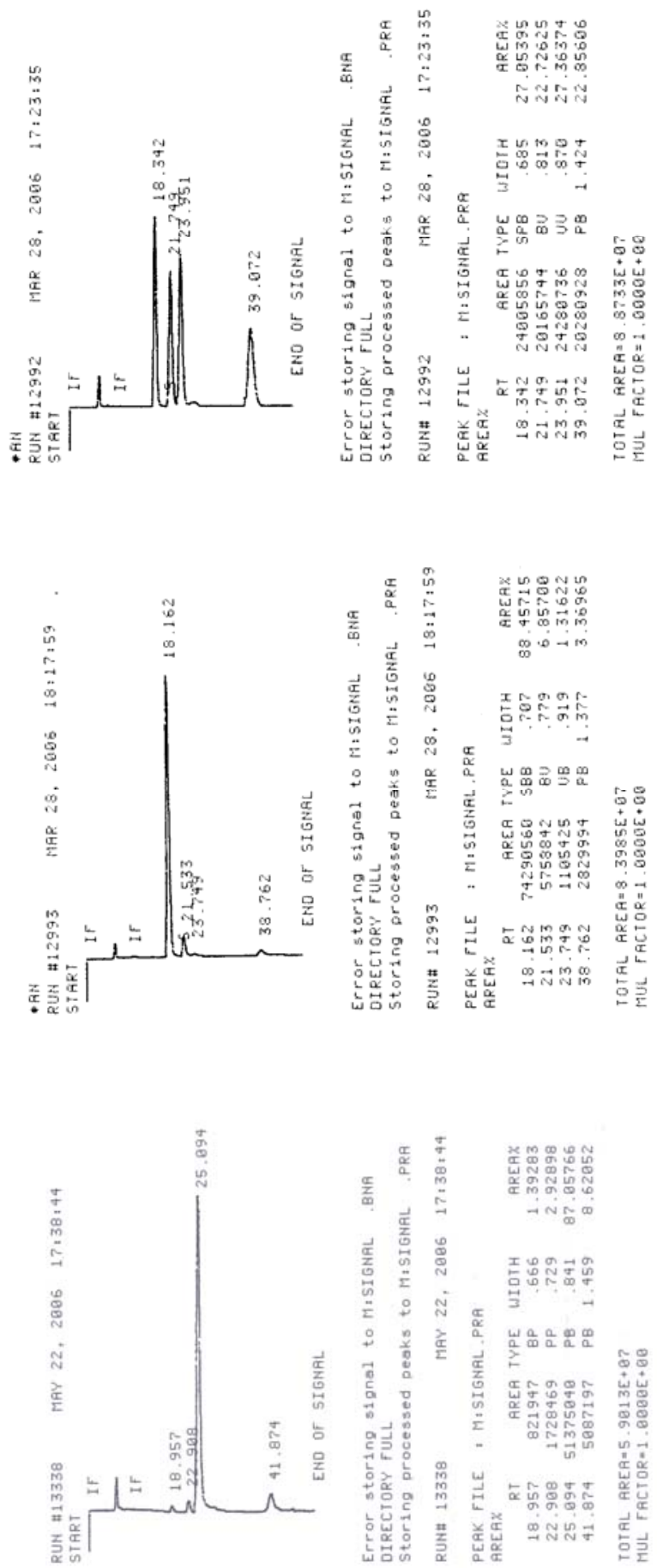
HPLC conditions for ee determination: $(\mathrm{R}, \mathrm{R})-$ Whelk-O 1, hexanes: isopropanol = 98: 2, $0.6 \mathrm{~mL} / \mathrm{min}, 220 \mathrm{~nm}$.<smiles>CC1(CC(Cl)C#N)CCc2ccccc2C1=O</smiles>

Racemic-5b<smiles>N#CC(Cl)C[C@]1(C#N)CCc2ccccc2C1=O</smiles>

$5 b$

99\% ee, product obtained with Q-2c<smiles>N#CC(Cl)C[C@]1(C#N)CCc2ccccc2C1=O</smiles>

97\% ee, product obtained With QD-2c
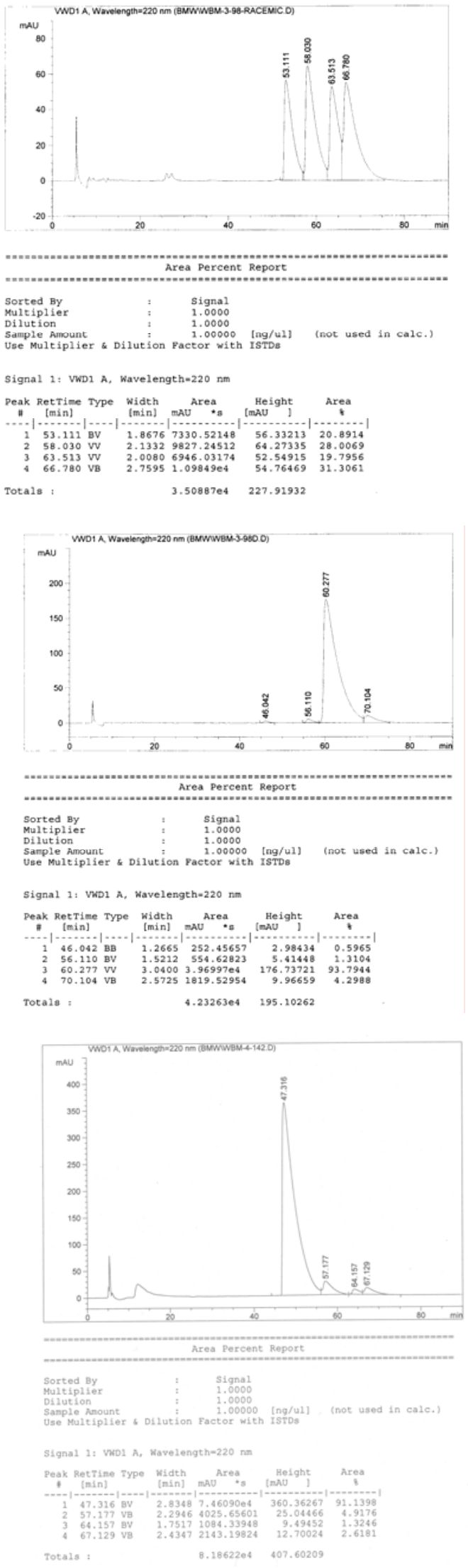
HPLC conditions for ee determination: Chiralcel OD, hexanes: isopropanol $=90: 10,1.0$ $\mathrm{mL} / \mathrm{min}, 220 \mathrm{~nm}$.<smiles>N#CC(Cl)CC1(C#N)CCCc2ccccc2C1=O</smiles>

Racemic-5c<smiles>N#CC(Cl)C[C@]1(C#N)CCCc2ccccc2C1=O</smiles>

97\% ee, product obtained with Q-2c<smiles>N#CC(Cl)C[C@]1(C#N)CCCc2ccccc2C1=O</smiles>

ent-5c

96\% ee, product obtained with QD-2c
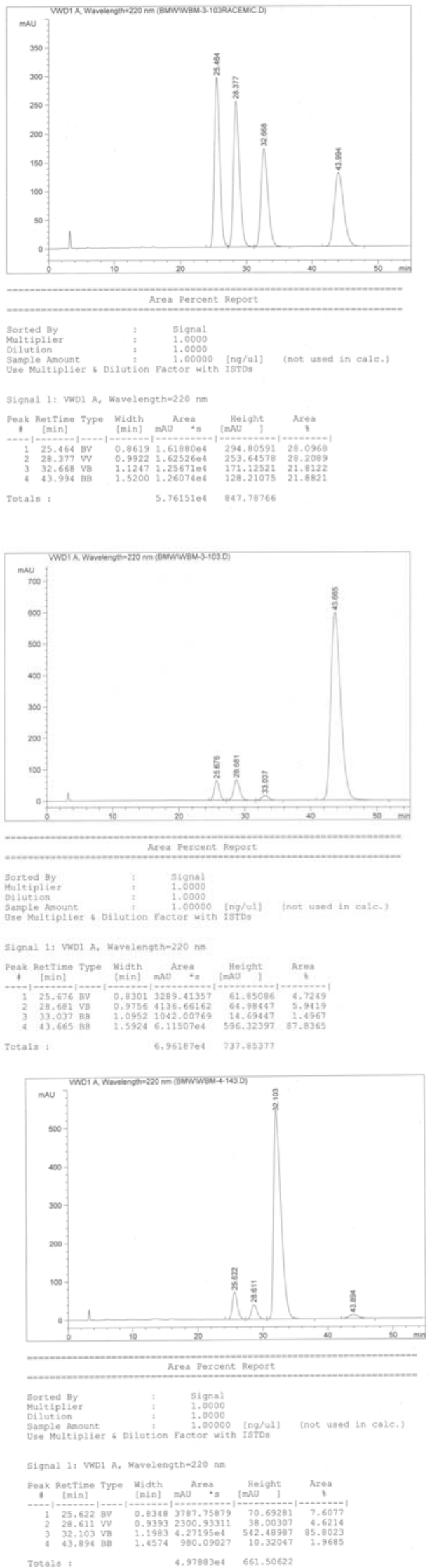

S 31 
GC conditions for ee determination: $\beta-\mathrm{CD}, 100{ }^{\circ} \mathrm{C}, 30 \mathrm{~min}$, then $100{ }^{\circ} \mathrm{C}-200{ }^{\circ} \mathrm{C}(0.5$ $\left.{ }^{\circ} \mathrm{C} / \mathrm{min}\right)$.<smiles>N#CC(Cl)CC1(C#N)CCCCCC1=O</smiles>

Racemic-5d
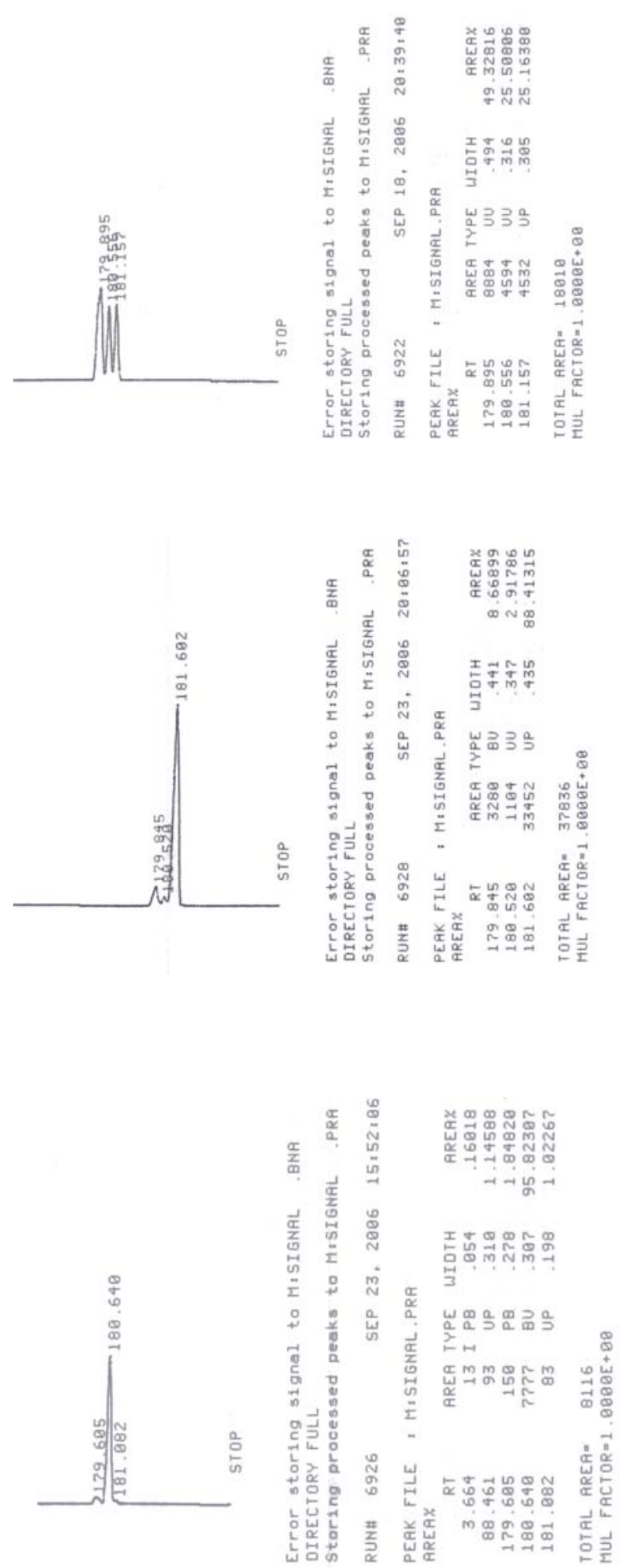
HPLC conditions for ee determination: Chiralcel OJ, hexanes: isopropanol $=70: 30,1.0$ $\mathrm{mL} / \mathrm{min}, 220 \mathrm{~nm}$.
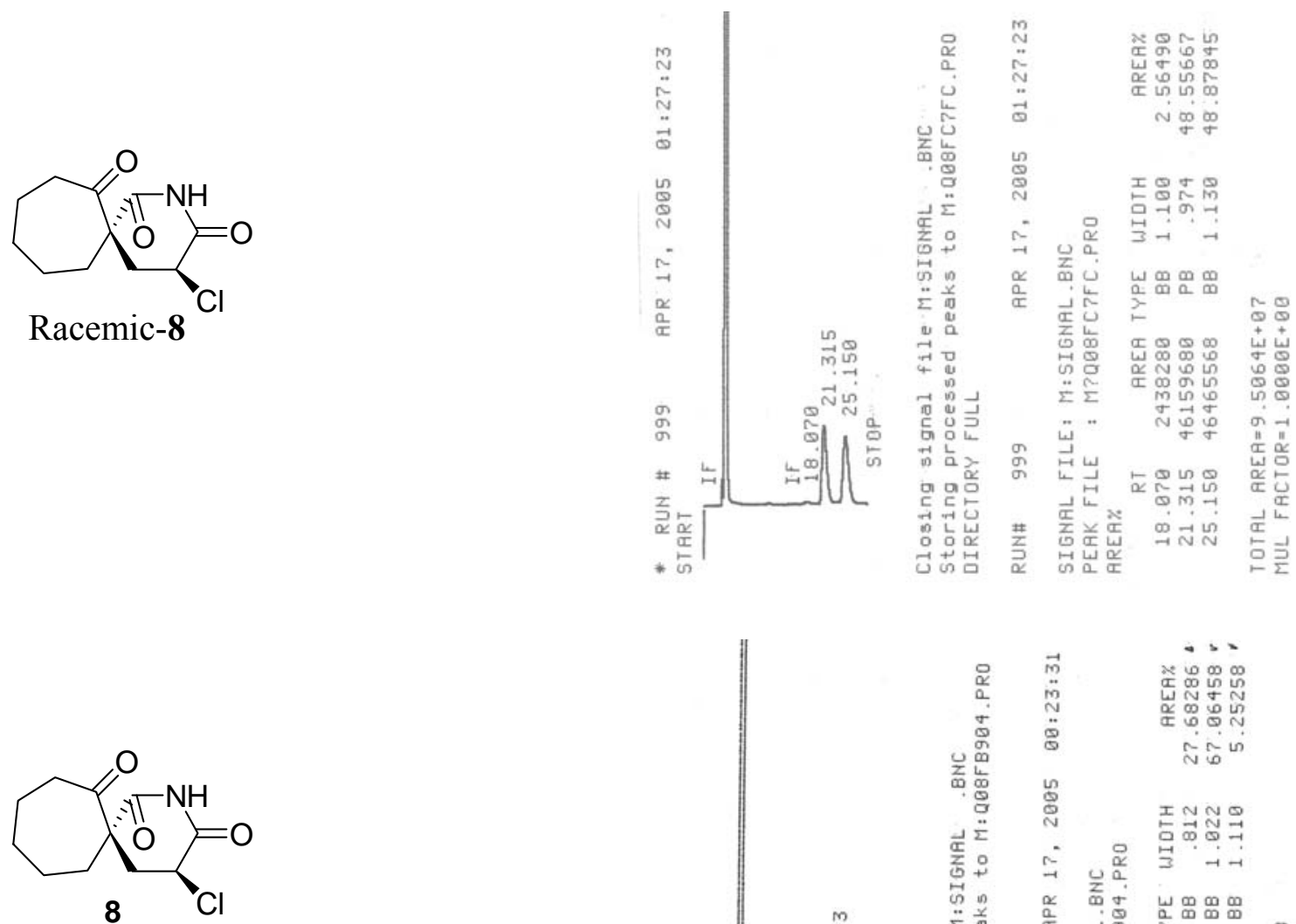

$85 \%$ ee, product from $\mathbf{5}$ 'd

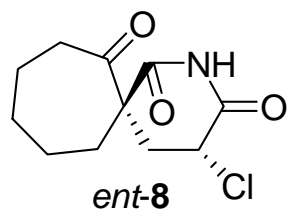

91\% ee, product from ent-5'd
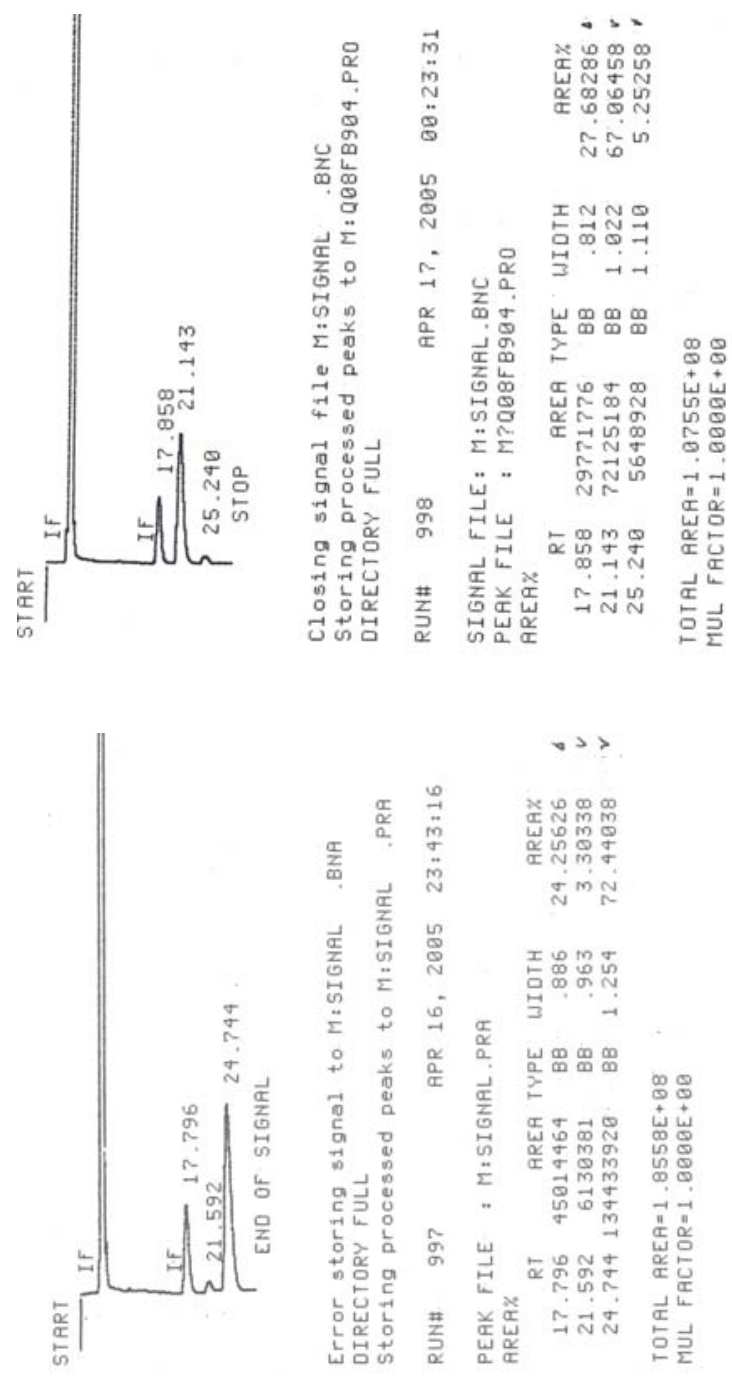
HPLC conditions for ee determination: Chiralcel OD, hexanes: isopropanol $=80: 20,1.0$ $\mathrm{mL} / \mathrm{min}, 220 \mathrm{~nm}$.

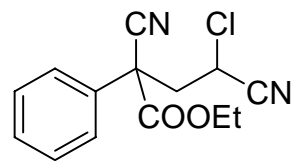

Racemic-5e
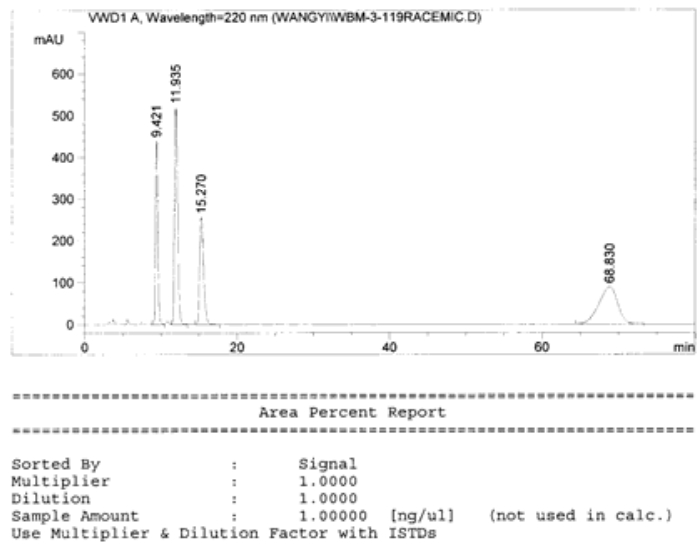

Sample Amount
Use Multiplier \& Dilution Factor with ISTDs

Signal 1: VWD1 $\mathrm{A}$, wavelength=220 nm

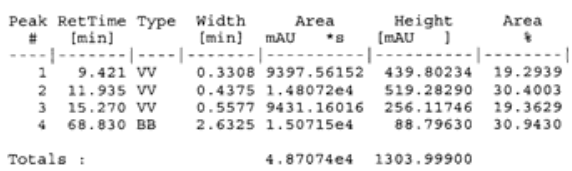

Totals : $\quad 4.87074 \mathrm{e} 4 \quad 1303.99900$
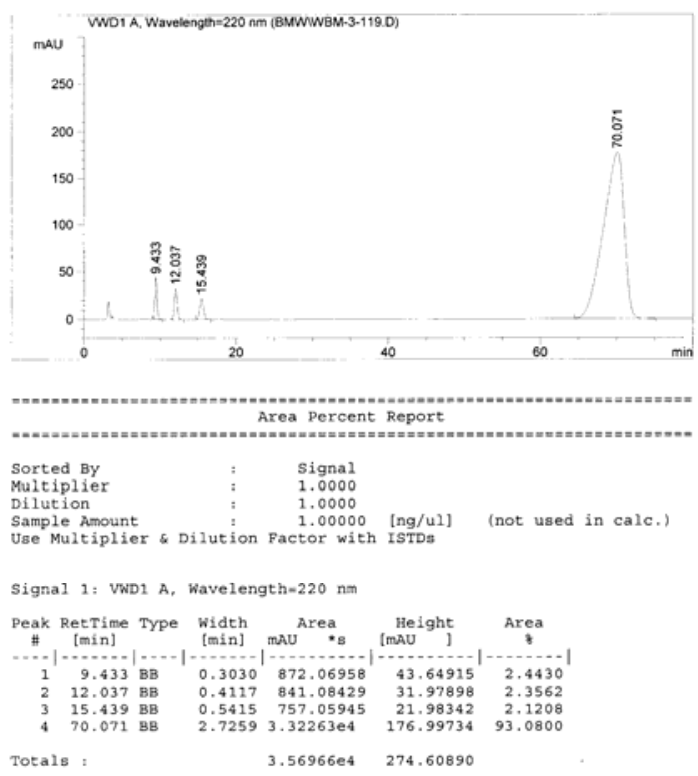
<smiles>CCOCC(C#N)(CC(Cl)Cl)c1ccccc1</smiles>

$95 \%$ ee, product obtained with QD-2c

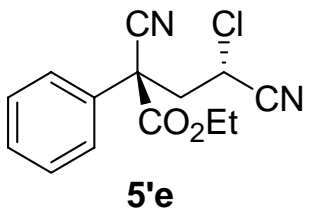

$86 \%$ ee, product obtained with Q-1b
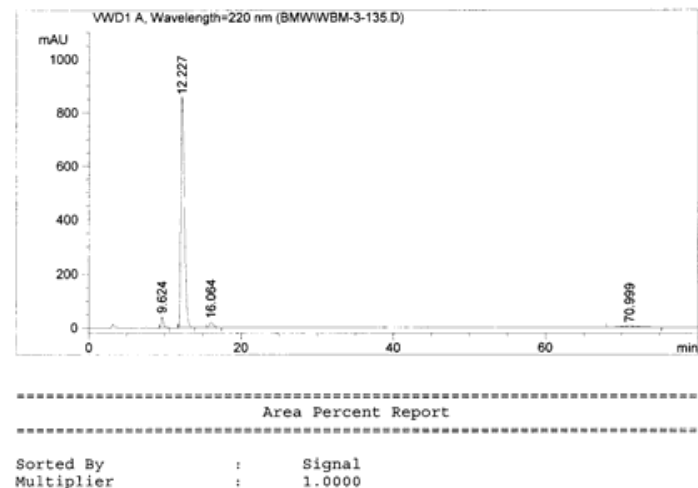

$\begin{array}{lll}\text { Sorted By } & \vdots & \text { Signal } \\ \text { Multiplier } & 1.0000\end{array}$

$\begin{array}{lclll}\text { Dilution } & 1.0000 & & \\ \text { Sample Amount } & 1.00000 & \text { [ng/ul] (not used in calc.) }\end{array}$

Signal 1: VWD1 A, wavelength $=220 \mathrm{~nm}$

Peak RetTime Type width Area
\# $[\mathrm{min}]$
$[\mathrm{min}]$
maU $* \mathrm{meight}$ Area

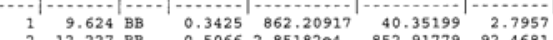

$\begin{array}{llllll}4 & 70.999 & \text { BB } & 2.5018 & 709.18103 & 4.20853\end{array}$

Totals : 3.08411 e4 917.57365

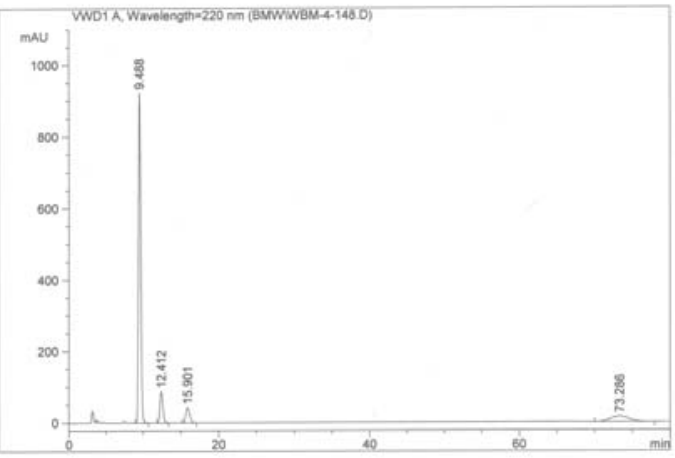

Area Percent Report

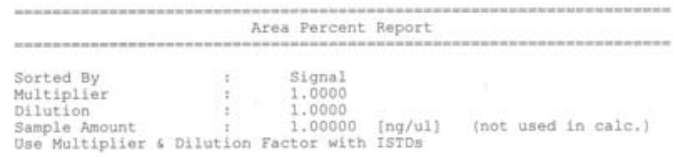

Signal 1: VWD1 $\AA$, Wavelength $220 \mathrm{~nm}$

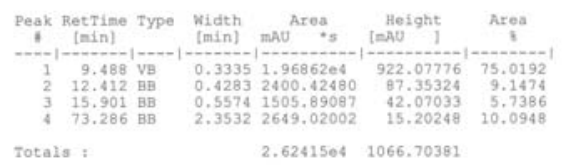


HPLC conditions for ee determination: Chiralcel OD, hexanes: isopropanol = 90: 10, 1 $\mathrm{mL} / \mathrm{min}, 220 \mathrm{~nm}$.

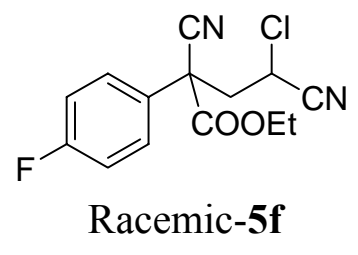

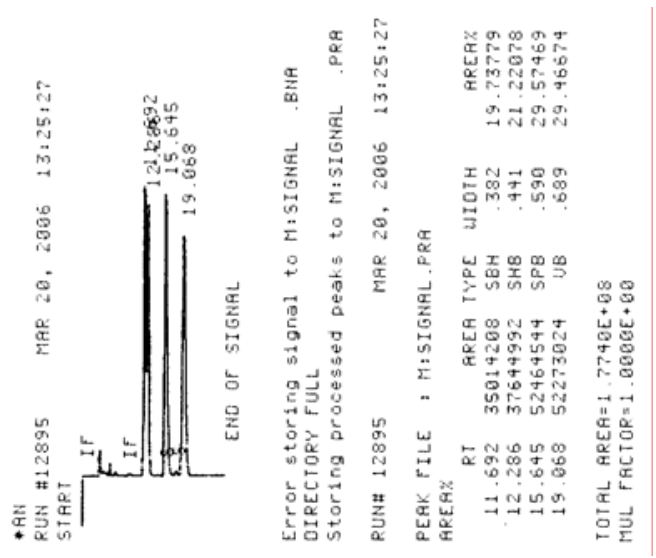<smiles>CCO[C@](C#N)(CC(Cl)C#N)c1ccc(F)cc1</smiles>

$95 \%$ ee, product obtained with Q-2c

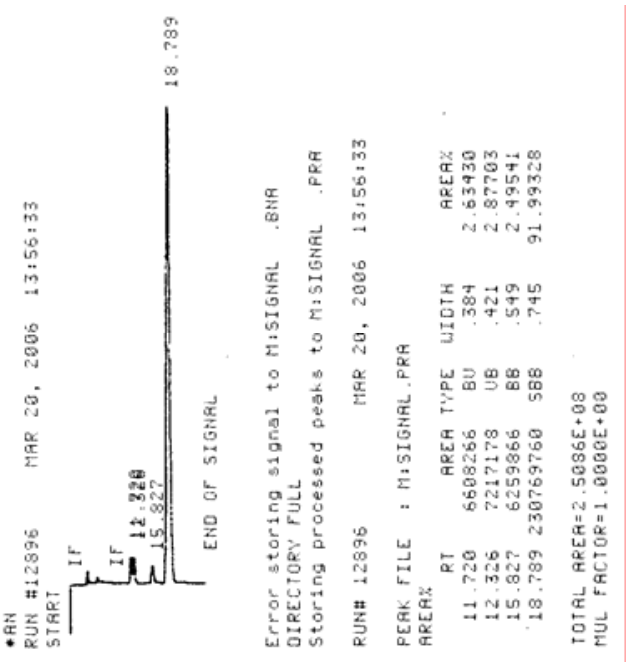<smiles>CCOCC(C#N)(CC(Cl)Cl)c1ccc(F)cc1</smiles>

$95 \%$ ee, product obtained with QD-2c

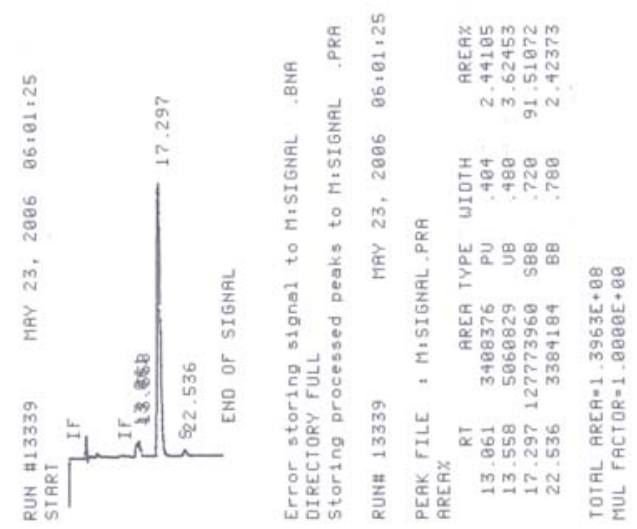


HPLC conditions for ee determination: Chiralcel OD, hexanes: isopropanol $=80: 20,1$ $\mathrm{mL} / \mathrm{min}, 220 \mathrm{~nm}$.<smiles>CCOC(C)(CC(C#N)C(Cl)Cl)c1ccc(Cl)cc1</smiles>

Racemic-5g<smiles>CC(Cl)CC(C#N)(c1ccc(Cl)cc1)C(Cl)C#N</smiles>

94\% ee, product obtained with Q-2c

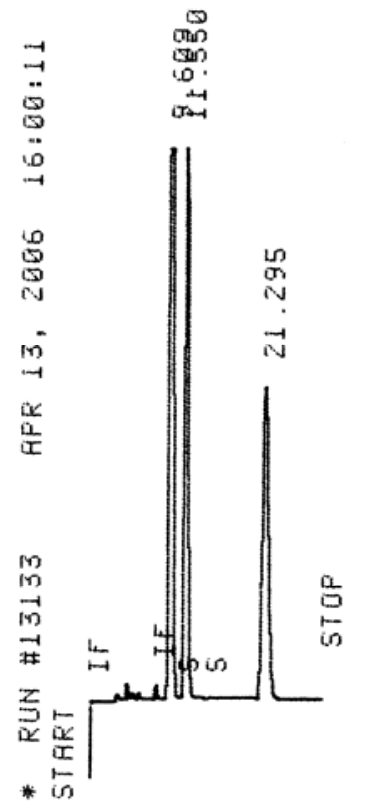

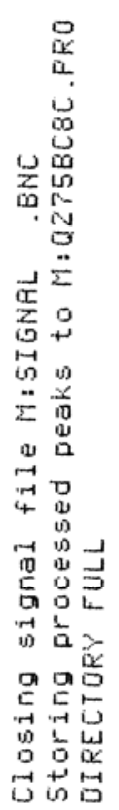

$\exists$
$\exists$
$\Xi$
$\Xi$
-1

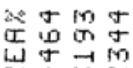

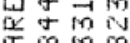

$\operatorname{gin}$

$\stackrel{\circ}{\mathscr{S}}$

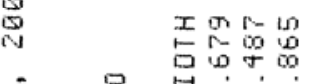

问最年

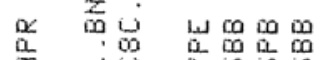

空思

$z_{0}$

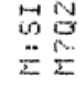

in تبـ

m

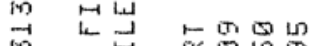

$\rightarrow$

\# 茫范

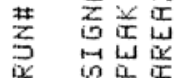

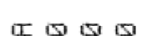

西品

舟

品罗

$\rightarrow$ on

II

品呚

咭与

䍃

到

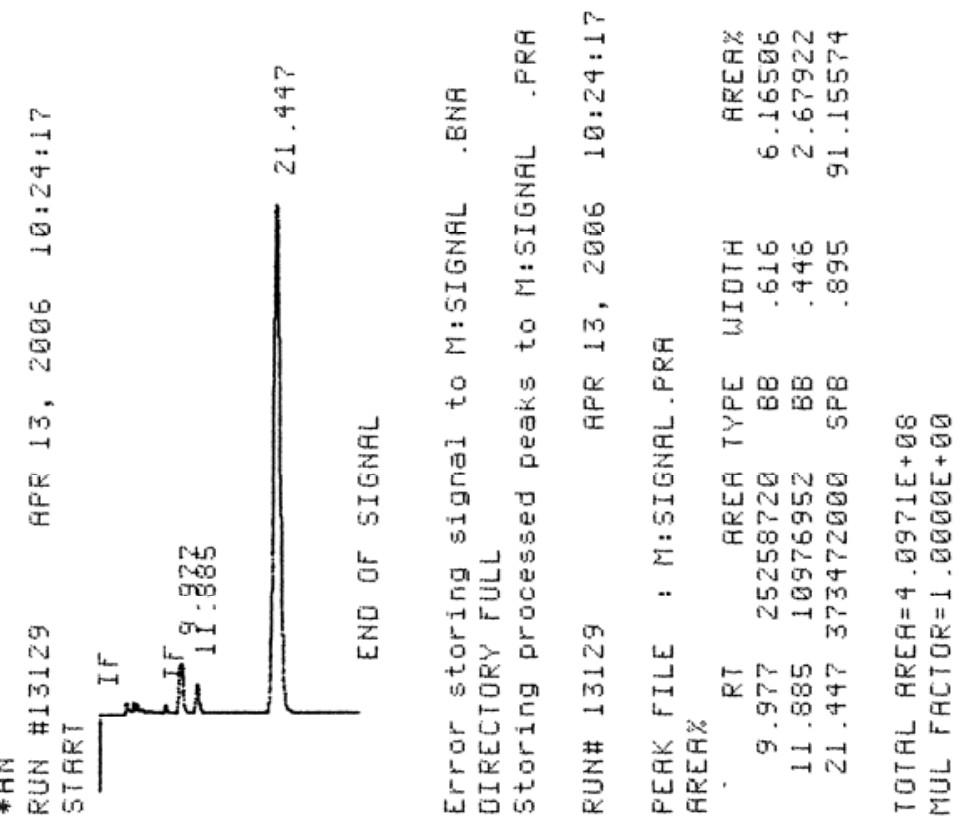


HPLC conditions: Chiralcel OD, hexanes: isopropanol =90: 10, $1.0 \mathrm{~mL} / \mathrm{min}, 220 \mathrm{~nm}$.<smiles>CCOC(=O)C(C#N)(CC(Cl)C#N)c1ccc(Br)cc1</smiles>

Racemic-5h<smiles>CCOC[C@@](C#N)(c1ccc(Br)cc1)[C@H](Cl)C#N</smiles>

95\% ee, product obtained with Q-2c<smiles>CCOCC(C#N)(c1ccc(Br)cc1)C(Cl)C#N</smiles>

94\% ee, product obtained With QD-2c
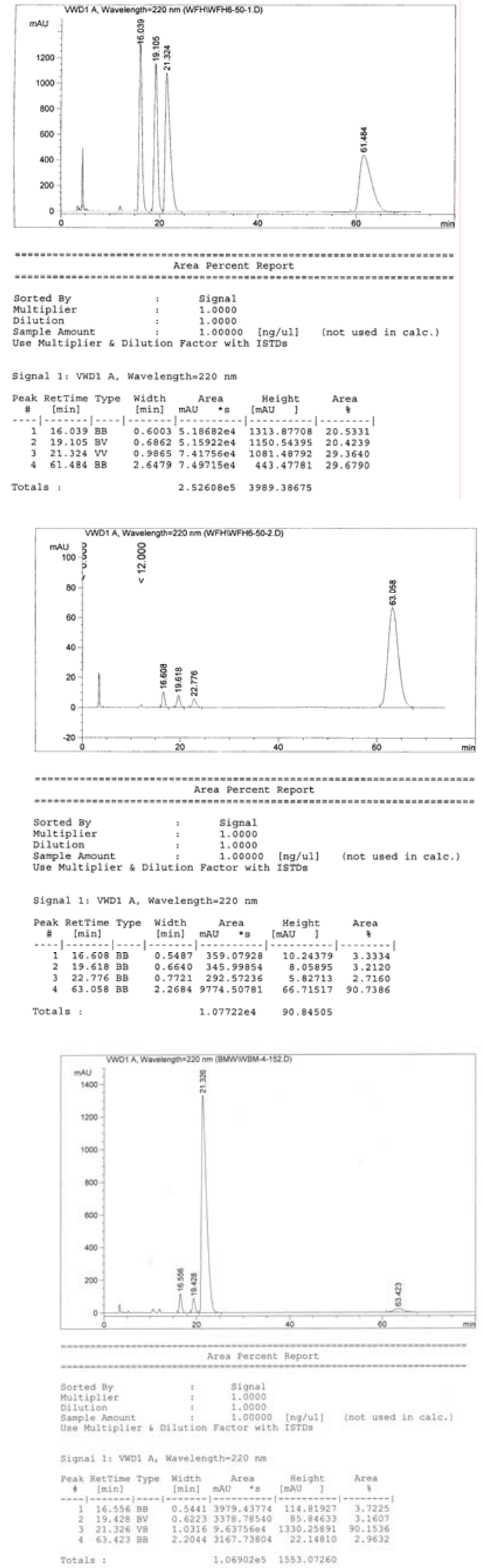
<smiles>CCOCC(C#N)(CC(Cl)Cl)c1ccc(Br)cc1</smiles>

$80 \%$ ee, product obtained with Q-1b<smiles>CCO[C@@H]([C@@H](Cl)C#N)[C@](C#N)(OCC)c1ccc(Br)cc1</smiles>

$86 \%$ ee, product obtained with QD-1b
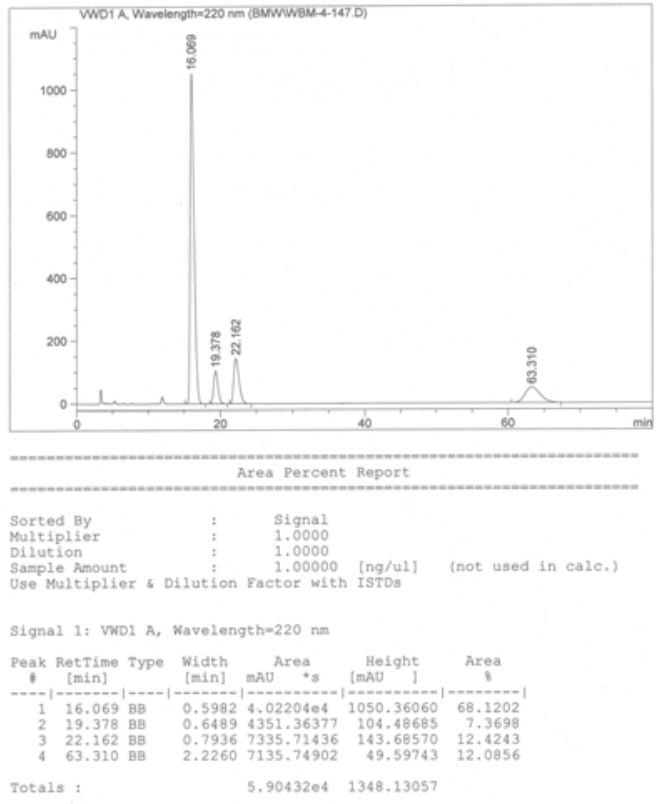

Totals

$\$ 9043204 \quad 1348.1305$

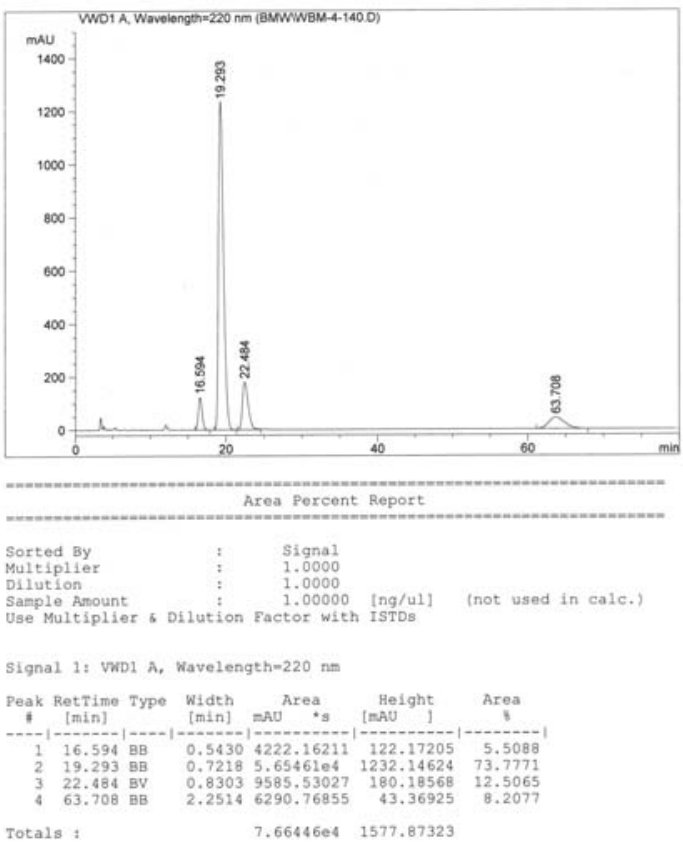


HPLC conditions for ee determination: Chiralcel OJ, hexanes: isopropanol $=60: 40,1.0$ $\mathrm{mL} / \mathrm{min}, 220 \mathrm{~nm}$.<smiles>CCOC(=O)C(C#N)(CC(Cl)Cl)c1ccc(C)cc1</smiles>

Racemic-5i<smiles>CCOC(=O)C(C#N)(CC(Cl)Cl)c1ccc(C)cc1</smiles>

95\% ee, product obtained with Q-2c
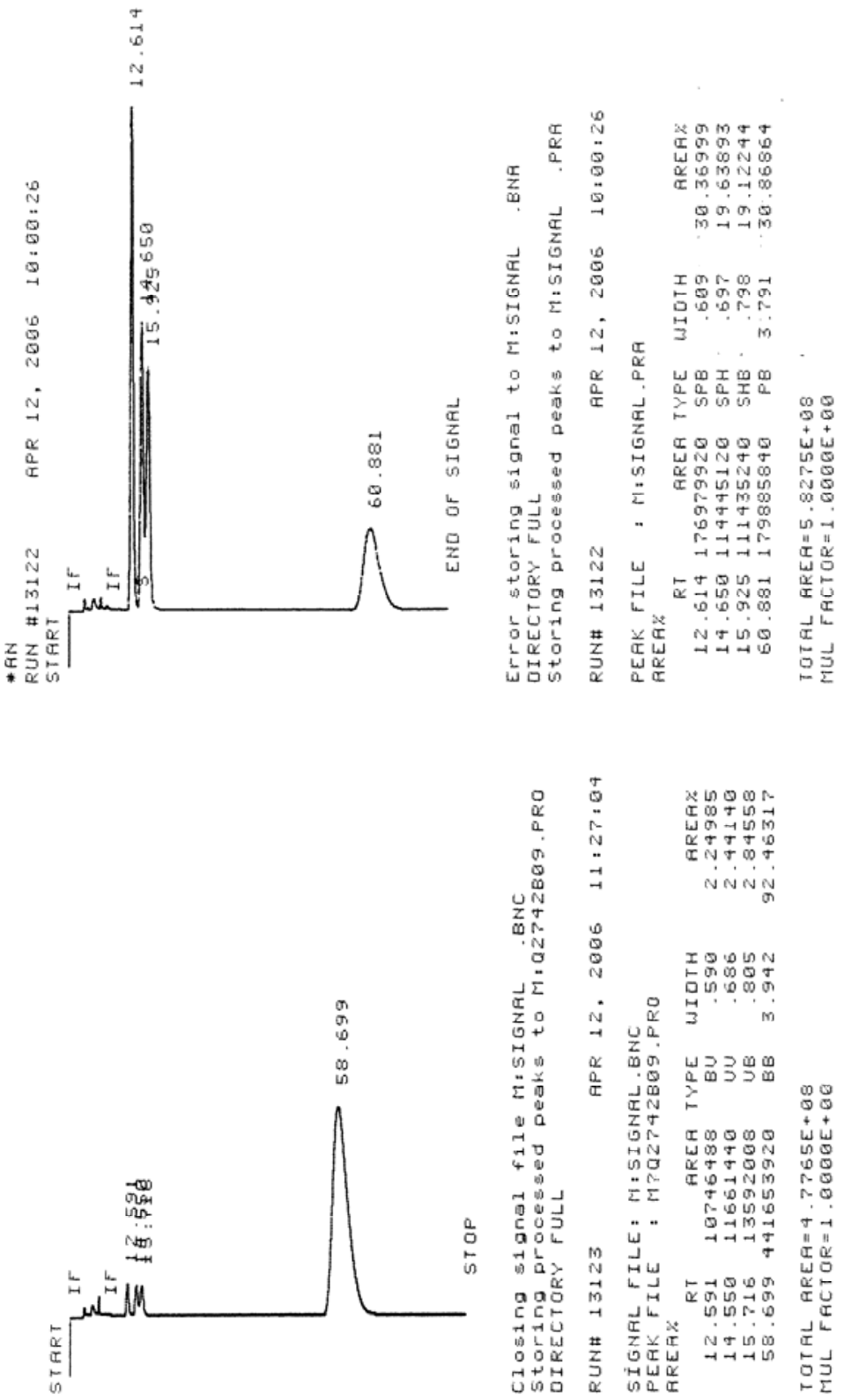
HPLC conditions for ee determination: Chiralcel OD, hexanes: isopropanol $=80: 20,1.0$ $\mathrm{mL} / \mathrm{min}, 220 \mathrm{~nm}$.<smiles>[R20]C#CCOC(C#N)(CC(Cl)Cl)c1ccc(OC)cc1</smiles><smiles>CCOC(=O)C(C#N)(c1ccc(OC)cc1)C(Cl)C#N</smiles>

95\% ee, product obtained with Q-2c<smiles>CCOC(C#N)(CC(Cl)Cl)c1ccc(OC)cc1</smiles>

95\% ee, product obtained with QD-2c
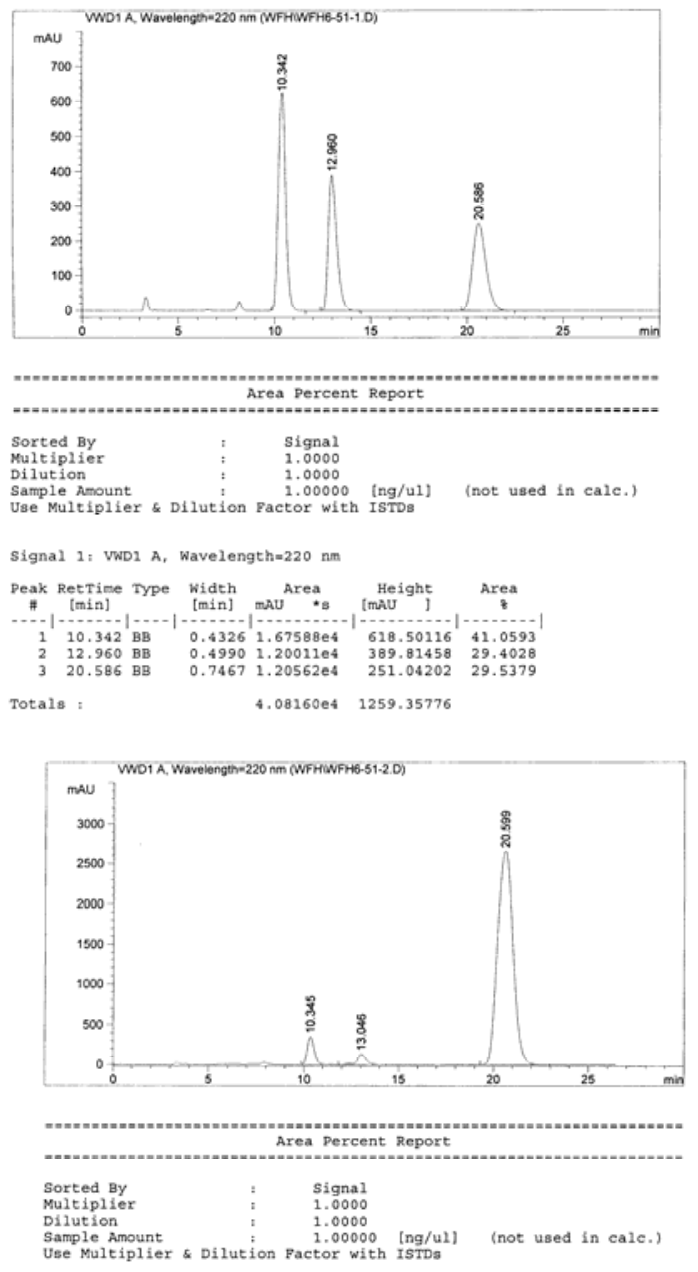

Signal 1: WWD1 A, wavelength=220 nm

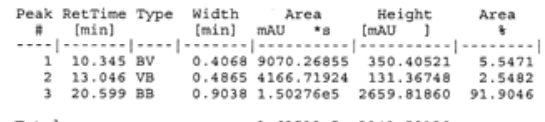

Totals : $\quad 1.63513 \mathrm{es} \quad 3141.59129$
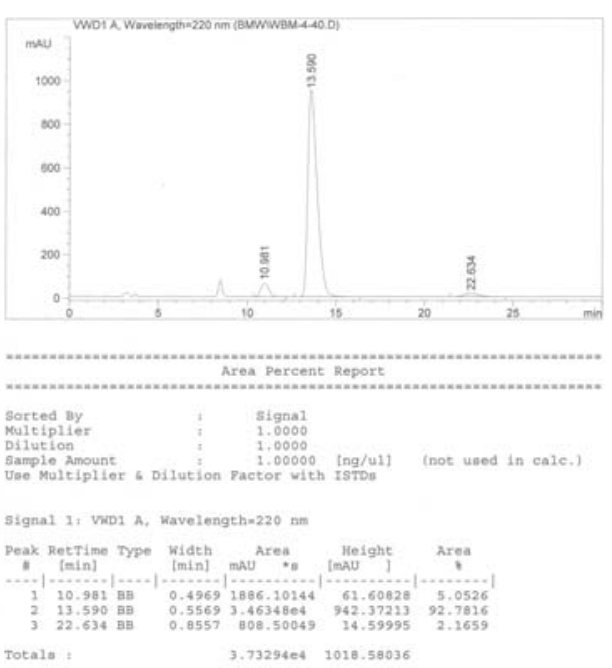

S 41 
HPLC conditions for ee determination: Chiralcel OD, hexanes: isopropanol $=80: 20,1.0$ $\mathrm{mL} / \mathrm{min}, 254 \mathrm{~nm}$.

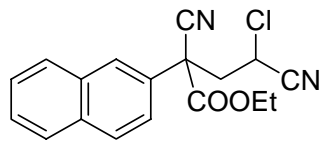

Racemic-5k<smiles>CCOC(C(Cl)Cl)C(C#N)c1ccc2ccccc2c1</smiles>

$5 \mathrm{k}$
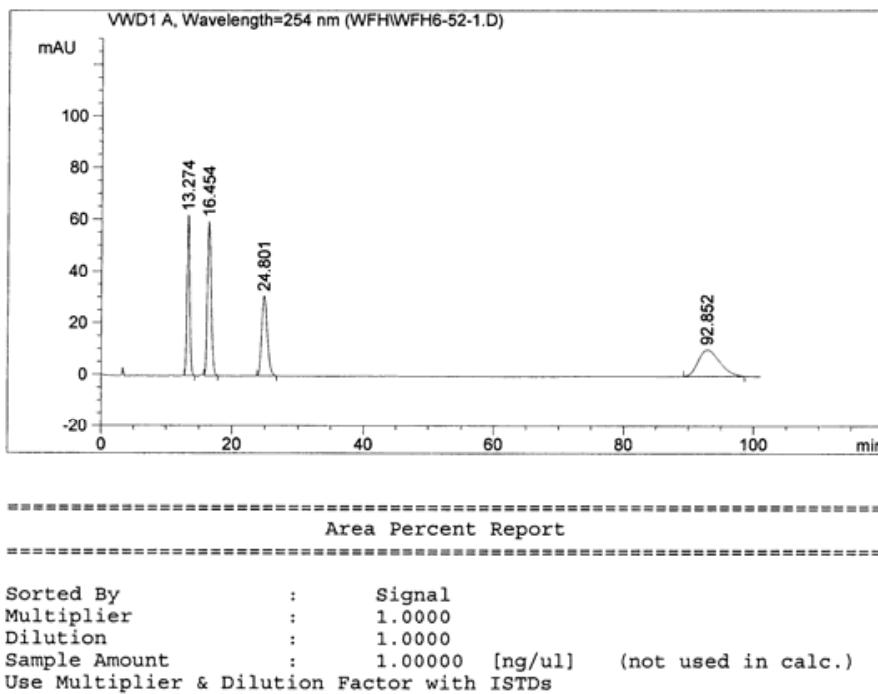

Signal 1: VWD1 A, Wavelength=254 nm

\begin{tabular}{|c|c|c|c|c|c|c|}
\hline Peak & RetTime & Type & Width & Area & Height & Area \\
\hline \# & [min] & & [min] & $\mathrm{MAU} \quad * \mathrm{~s}$ & [mAU ] & 8 \\
\hline 1 & 13.274 & BB & 0.4863 & 1835.55566 & 61.79380 & 21.7461 \\
\hline 2 & 16.454 & BB & 0.6053 & 2378.25220 & 59.59758 & 28.1755 \\
\hline 3 & 24.801 & BB & 0.9067 & 1851.55505 & 30.98944 & 21.9356 \\
\hline 4 & 92.852 & BB & 3.5749 & 2375.48755 & 10.21559 & 28.1428 \\
\hline & & & & 8440.85046 & 162.59641 & \\
\hline
\end{tabular}
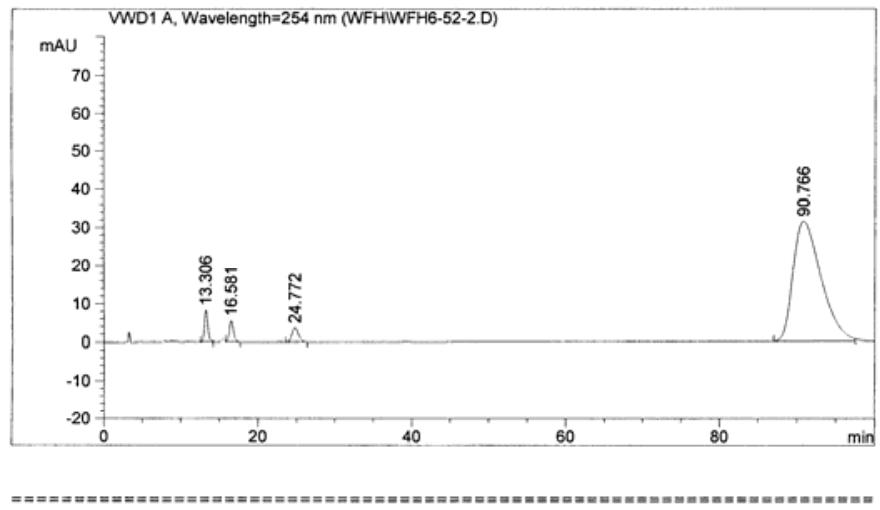
Area Percent Report

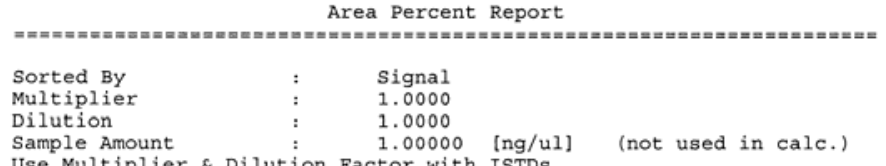

Sample Amount $\quad: \quad 1.00000$ lng/ul

Signal 1: VWD1 $A$, Wavelength $=254 \mathrm{~nm}$

\begin{tabular}{|c|c|c|c|c|c|c|}
\hline Peak & RetTime & Type & Width & Area & Height & Area \\
\hline \# & [min] & & [min] & $\mathrm{mAU} \quad * \mathrm{~s}$ & {$[\mathrm{mAU}]$} & 8 \\
\hline 1 & 13.306 & BB & 0.4963 & 251.94394 & 8.24400 & 2.9792 \\
\hline 2 & 16.581 & BB & 0.6105 & 207.61452 & 5.41361 & 2.4550 \\
\hline 3 & 24.772 & BB & 0.9043 & 208.52855 & 3.62368 & 2.4658 \\
\hline 4 & 90.766 & BB & 3.9090 & 7788.72803 & 31.25552 & 92.1000 \\
\hline & ${ }^{\circ}$ & & & 8456.81503 & 48.53681 & \\
\hline
\end{tabular}


HPLC conditions for ee determination: Chiralcel OD, hexanes: isopropanol $=90: 10,1.0$ $\mathrm{mL} / \mathrm{min}, 220 \mathrm{~nm}$.
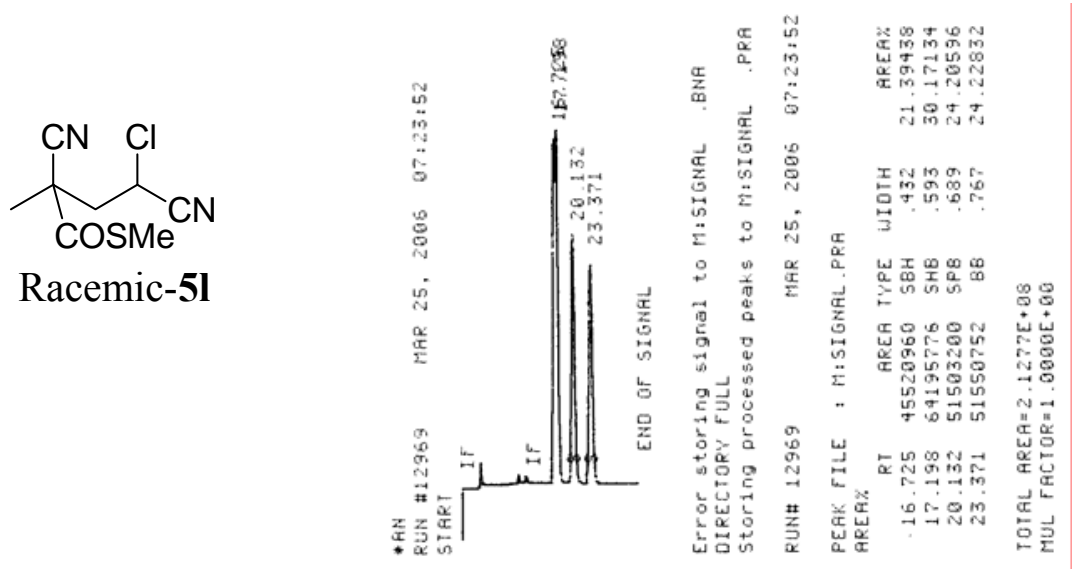

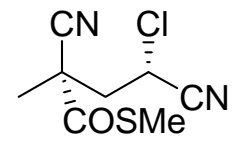

5I

$96 \%$ ee, product obtained with Q-2c

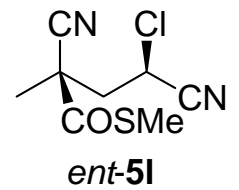

96\% ee, product obtained with QD-2c
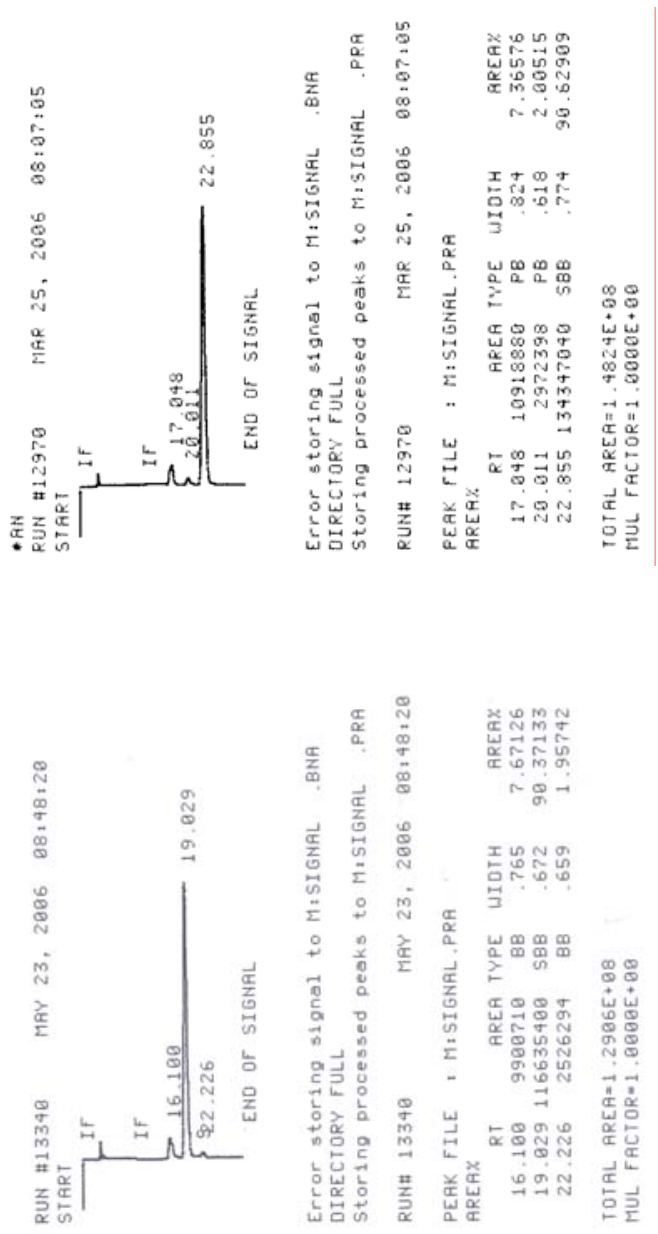
HPLC conditions for ee determination: Chiralcel OJ, hexanes: isopropanol $=70: 30,1.0$ $\mathrm{mL} / \mathrm{min}, 220 \mathrm{~nm}$.<smiles>COC(C)(C)CC(Cl)C#N</smiles>

Racemic-5l
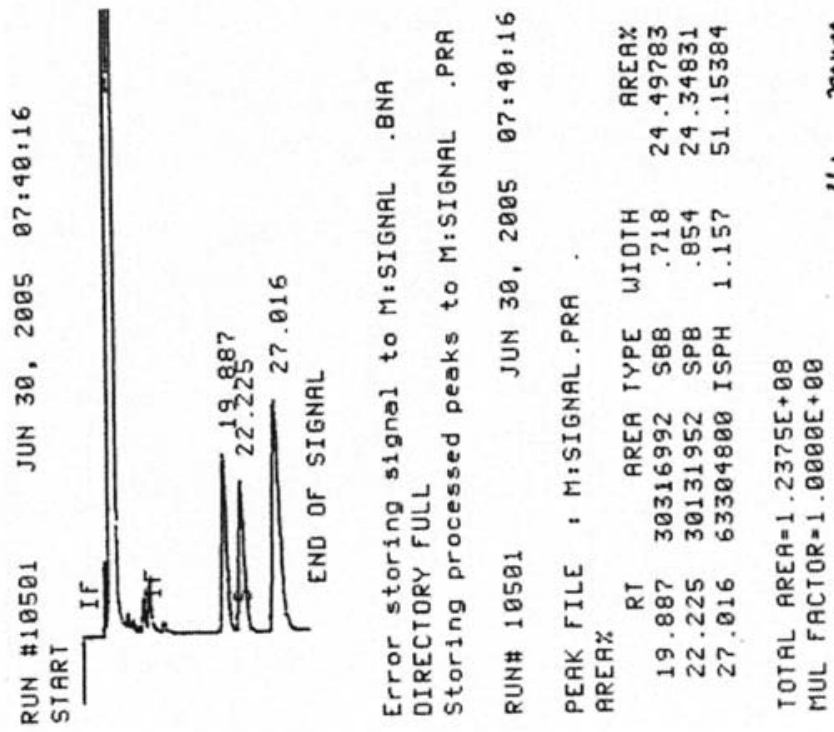

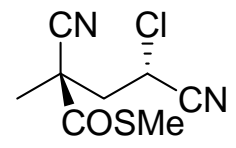

5'I

$88 \%$ ee, product obtained with Q-1b

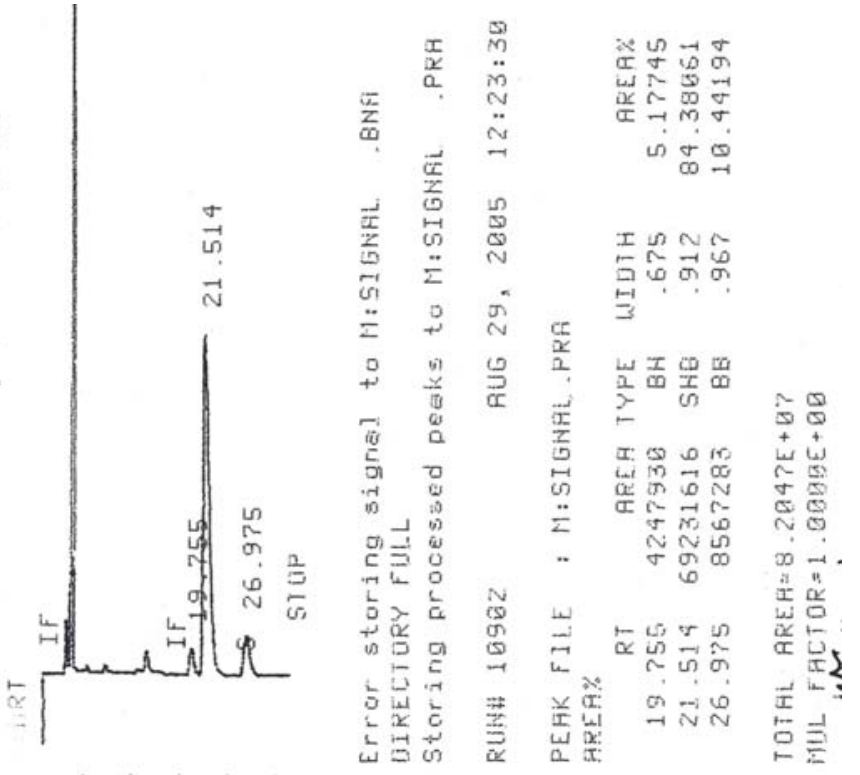


HPLC conditions for ee determination: Chiralcel OJ, hexanes: isopropanol $=96: 4,1.0$ $\mathrm{mL} / \mathrm{min}, 220 \mathrm{~nm}$.
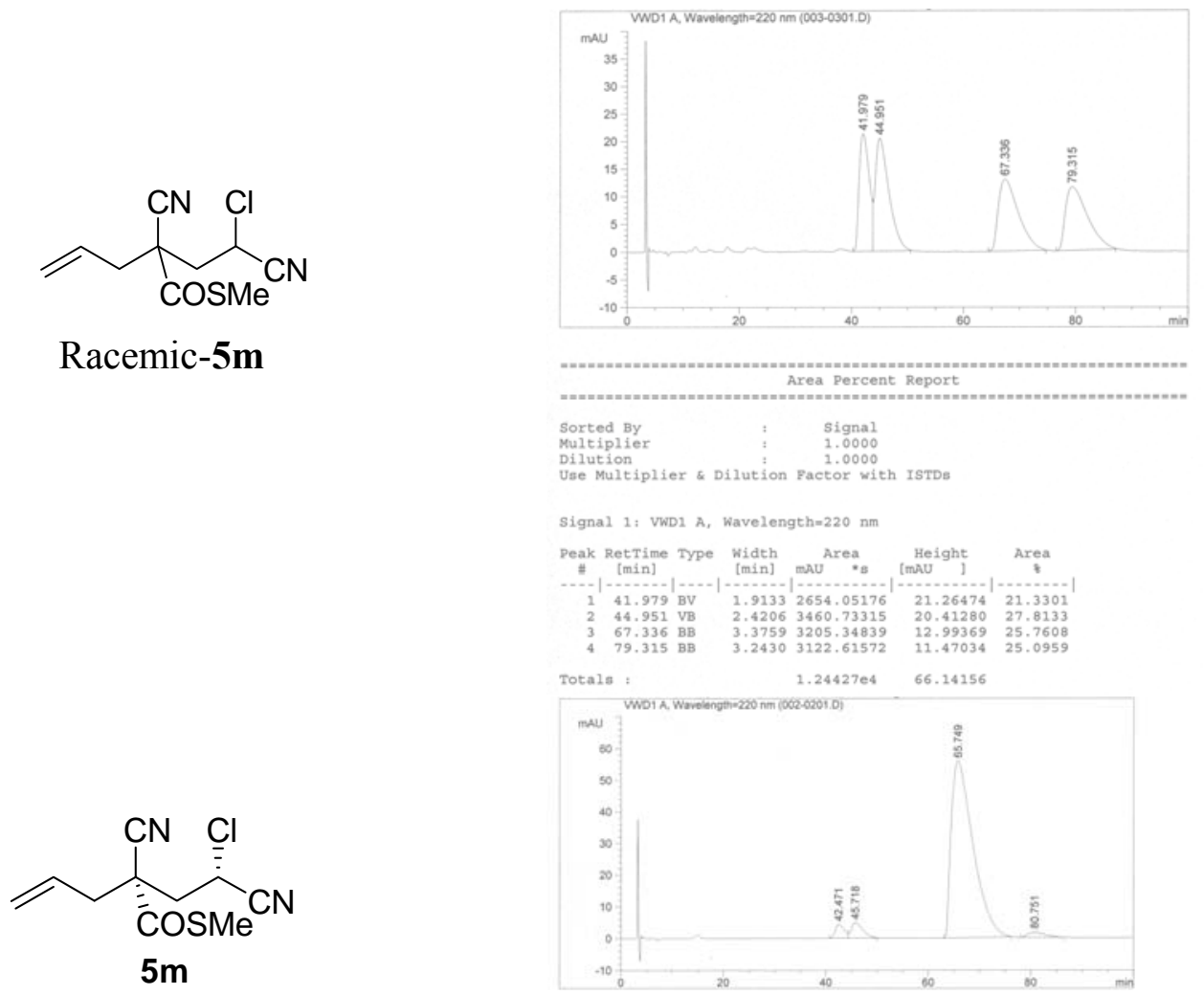

$95 \%$ ee, product obtained with Q-2c
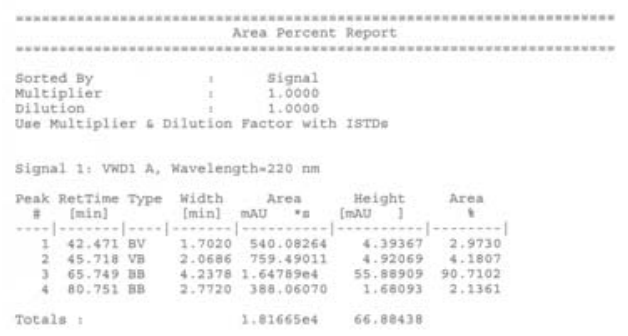

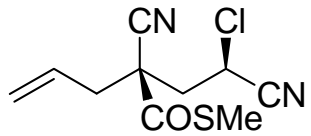

ent-5m

$95 \%$ ee, product obtained with QD-2c
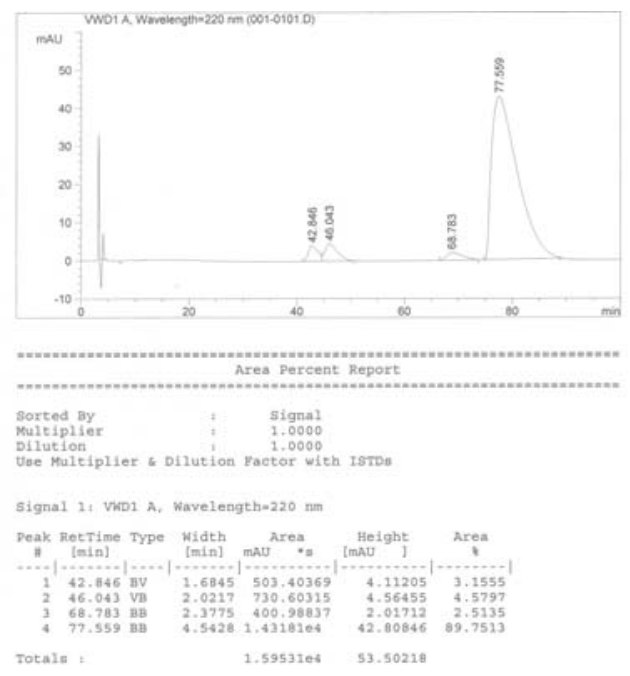

S 45 
HPLC conditions for ee determination: Chiralcel OD, hexanes: isopropanol $=60: 40,1.0$ $\mathrm{mL} / \mathrm{min}, 220 \mathrm{~nm}$.
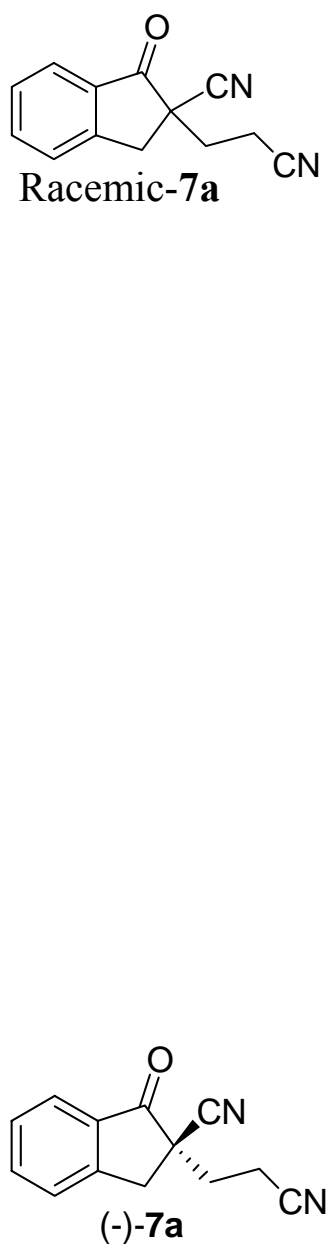

$93 \%$ ee, product obtained with Q-2c
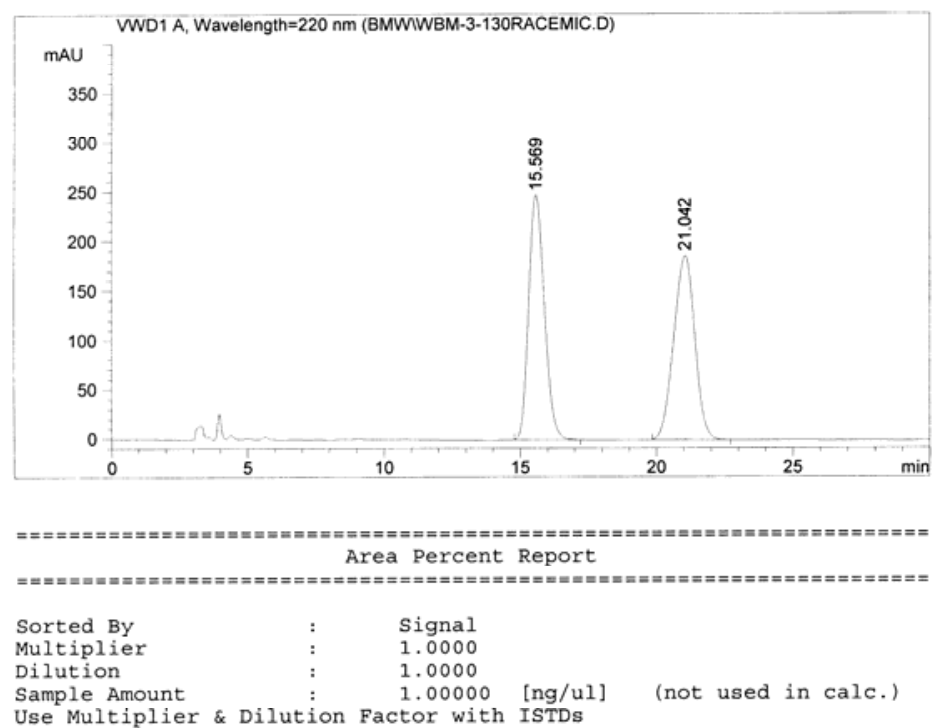

Signal 1: VWD1 $A$, wavelengt $h=220 \mathrm{~nm}$
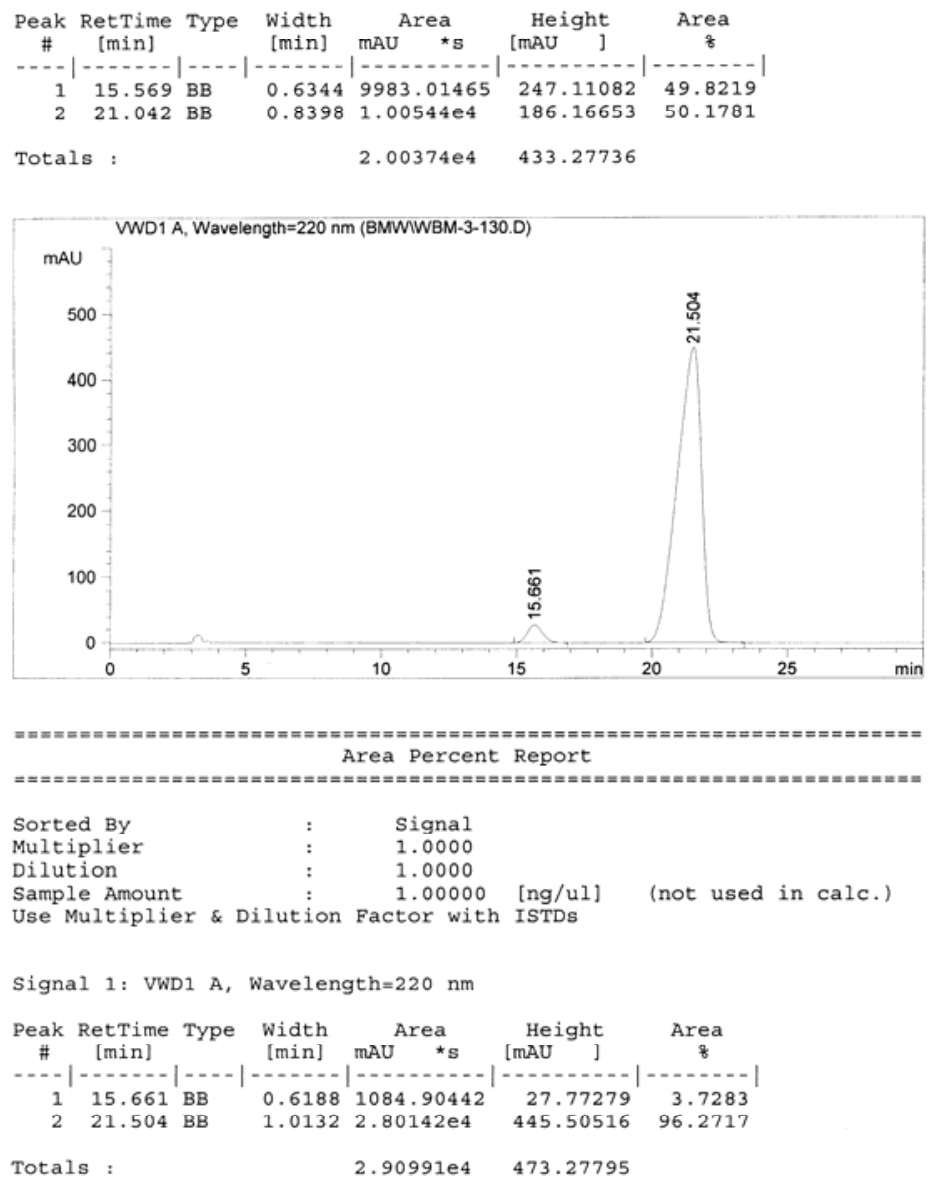
HPLC conditions for ee determination: Chiralcel OD, hexanes: isopropanol $=80: 20,1.0$ $\mathrm{mL} / \mathrm{min}, 220 \mathrm{~nm}$.

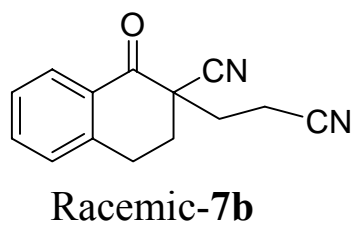

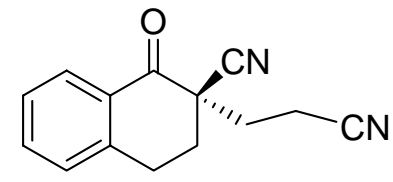

$(+)-7 b$

$94 \%$ ee, product obtained with Q-2c
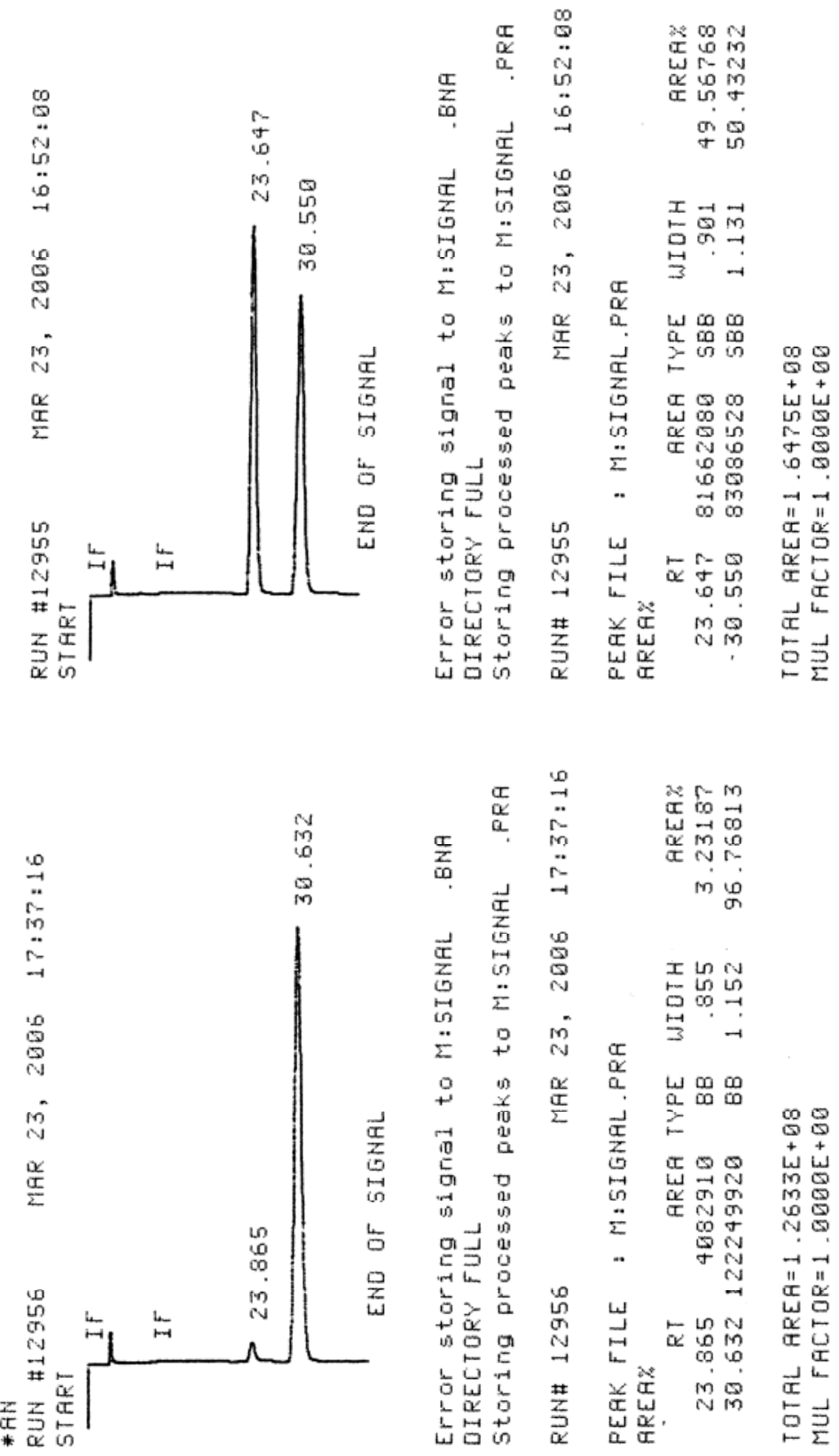
HPLC conditions for ee determination: Chiralcel OD, hexanes: isopropanol $=80: 20,1.0$ $\mathrm{mL} / \mathrm{min}, 220 \mathrm{~nm}$.<smiles>CCOC(=O)C(C#N)(CCC#N)c1ccccc1</smiles>

Racemic-7e<smiles>CCOC(=O)[C@](C#N)(CCC#N)c1ccccc1</smiles>

$(-)-7 e$

$89 \%$ ee, product obtained with Q-2c<smiles>CCOC[C@@](C#N)(CCC#N)c1ccccc1</smiles>

$(+)-7 e$

$90 \%$ ee, product obtained with QD-2c

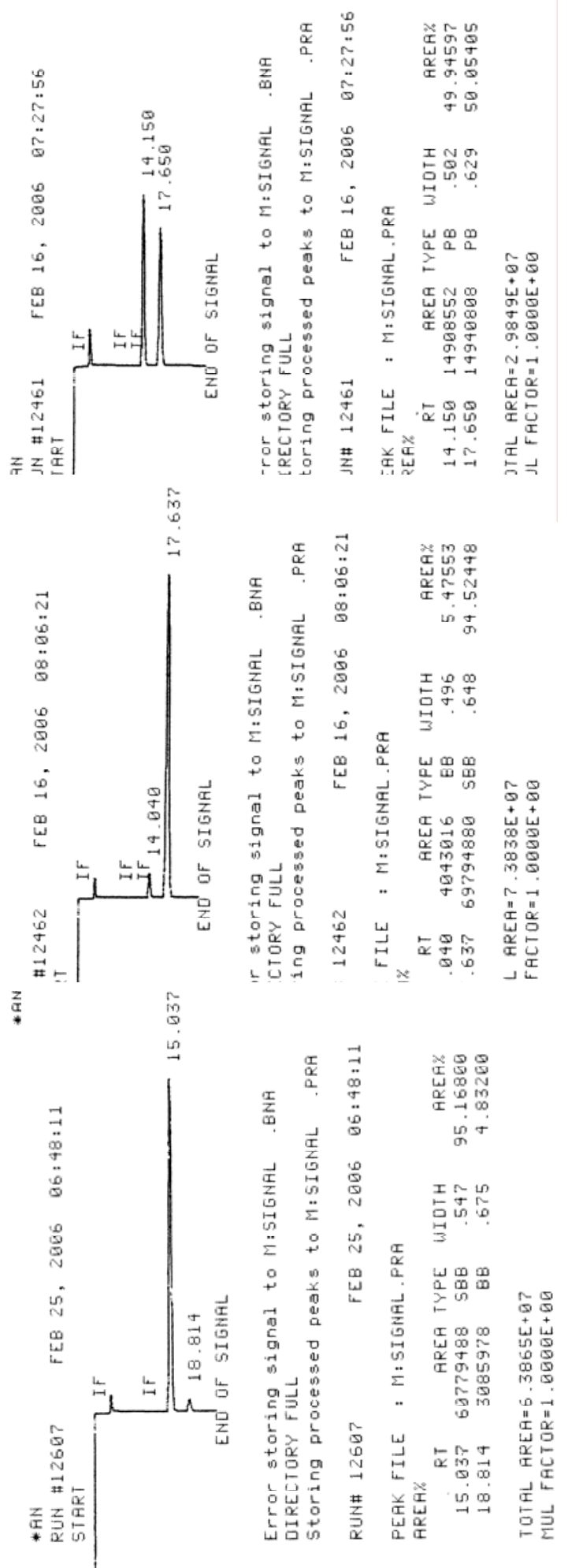


HPLC conditions for ee determination: Chiralpak AD, hexanes: isopropanol = 98: 2, 1.0 $\mathrm{mL} / \mathrm{min}, 220 \mathrm{~nm}$.<smiles>CCOC(=O)C(C)(CCC#N)c1ccc(F)cc1</smiles>

Racemic-7f

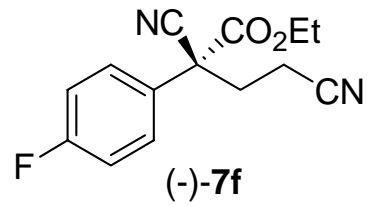

$88 \%$ ee, product obtained with Q-2c

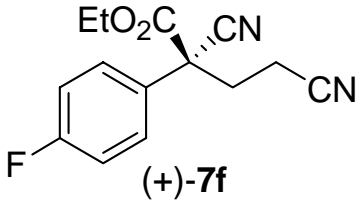

$90 \%$ ee, product obtained with QD-2c
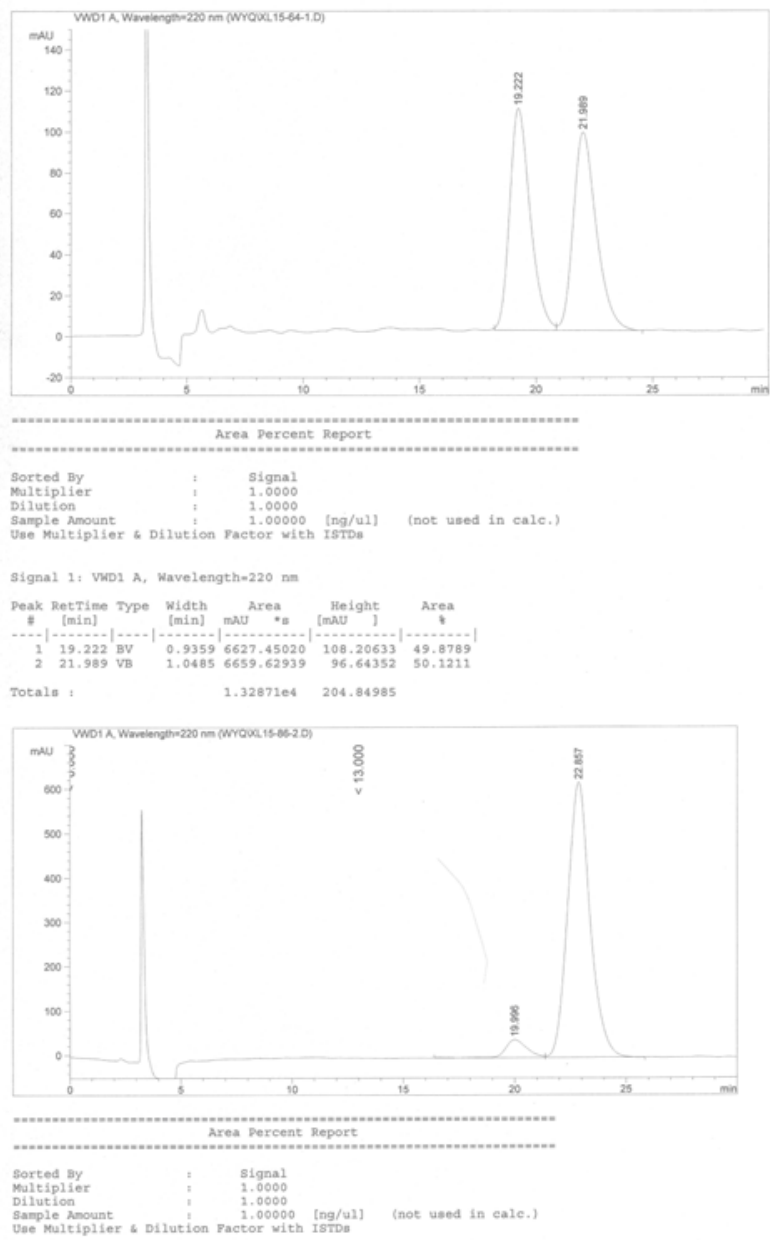

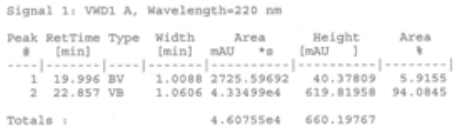

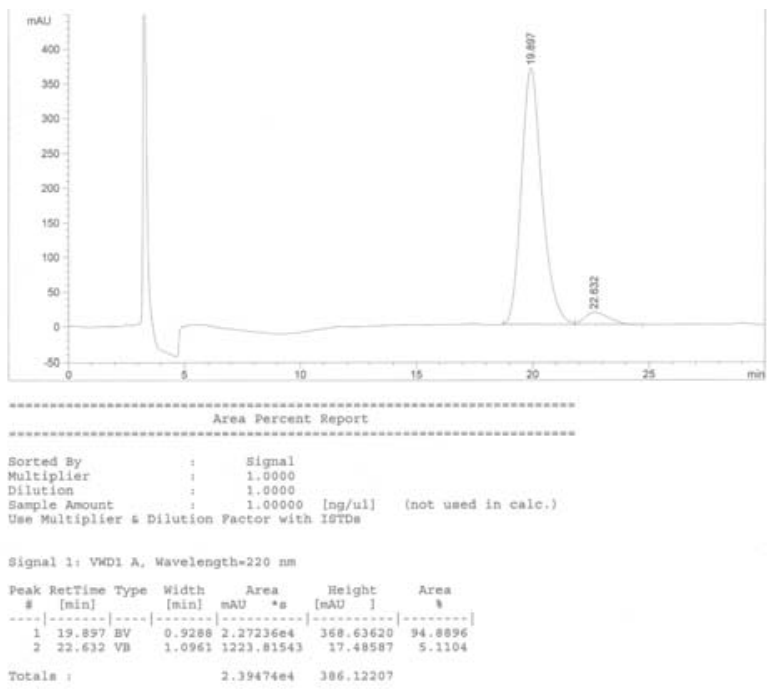


HPLC conditions for ee determination: Chiralcel OJ, hexanes: isopropanol $=60: 40,0.8$ $\mathrm{mL} / \mathrm{min}, 220 \mathrm{~nm}$.<smiles>[R4]C=CCCC(C)(CCC#N)c1ccc(Cl)cc1</smiles>

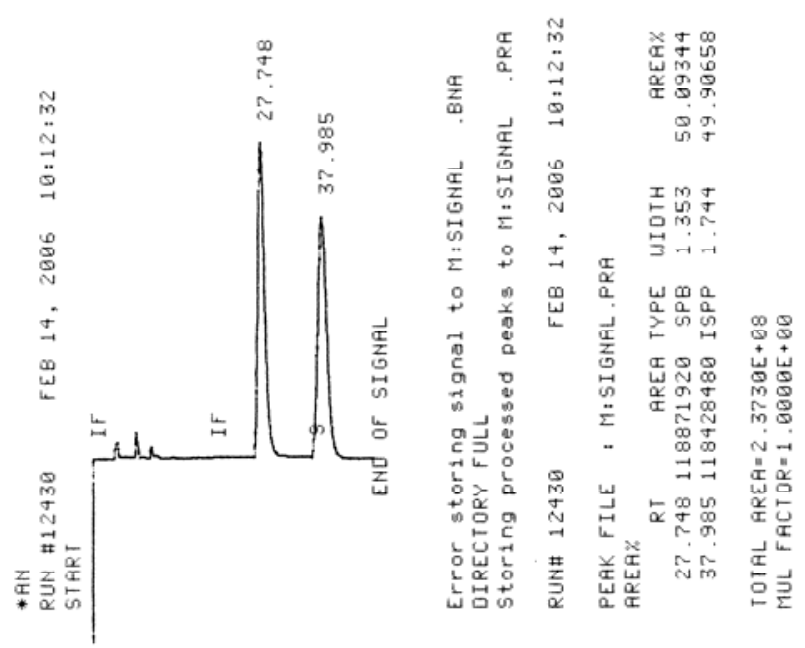<smiles>CCOC(=O)C(C#N)(CCC#N)c1ccc(Cl)cc1</smiles>

$88 \%$ ee, product obtained with Q-2c<smiles>N#CCCC(C#N)(CCCO)c1ccc(Cl)cc1</smiles>

$89 \%$ ee, product obtained with QD-2c
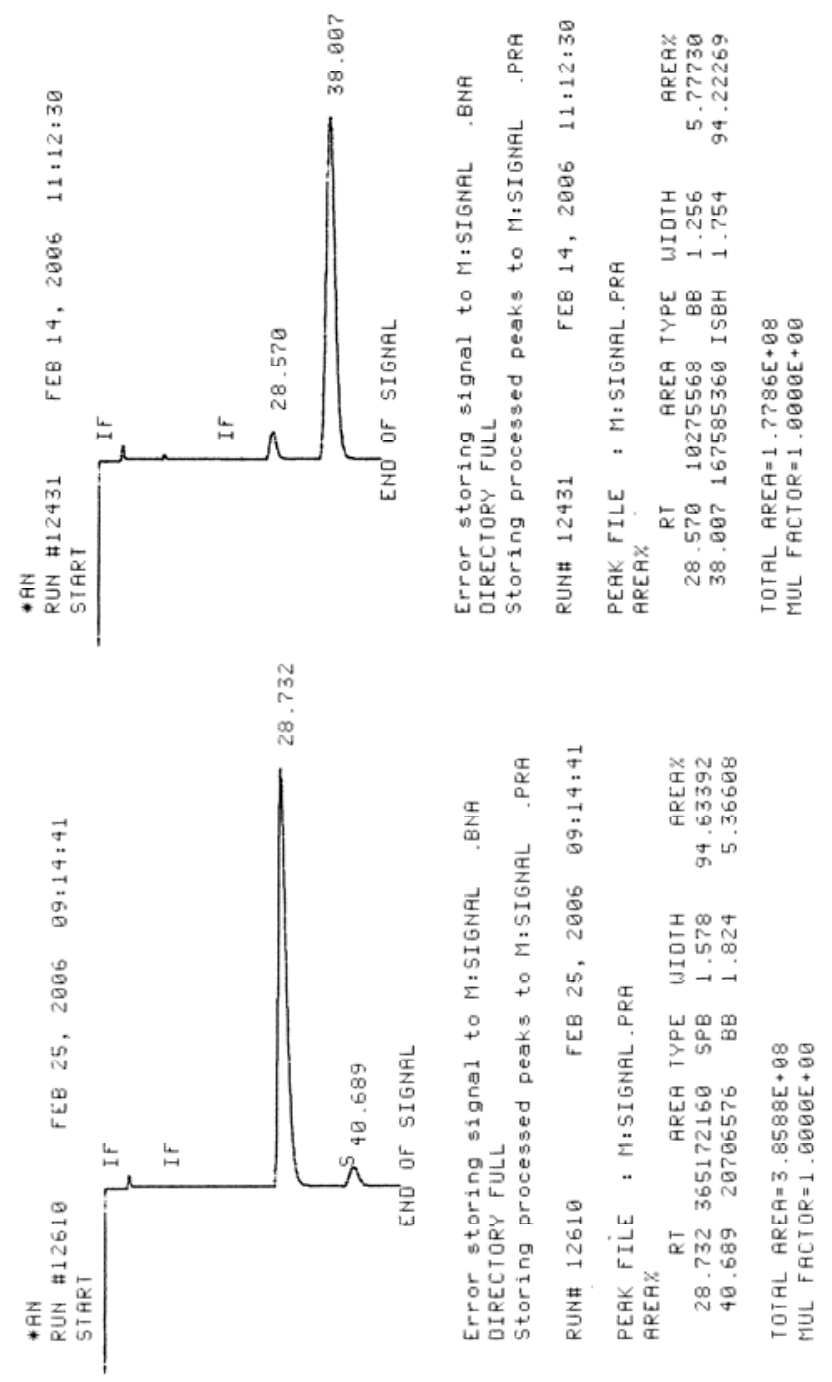
HPLC conditions for ee determination: $(\mathrm{R}, \mathrm{R})-$ Whelk-O 1, hexanes: isopropanol = 97: 3, $1.0 \mathrm{~mL} / \mathrm{min}, 220 \mathrm{~nm}$.<smiles>[R4]C([CH])[CH][CH][CH]</smiles>
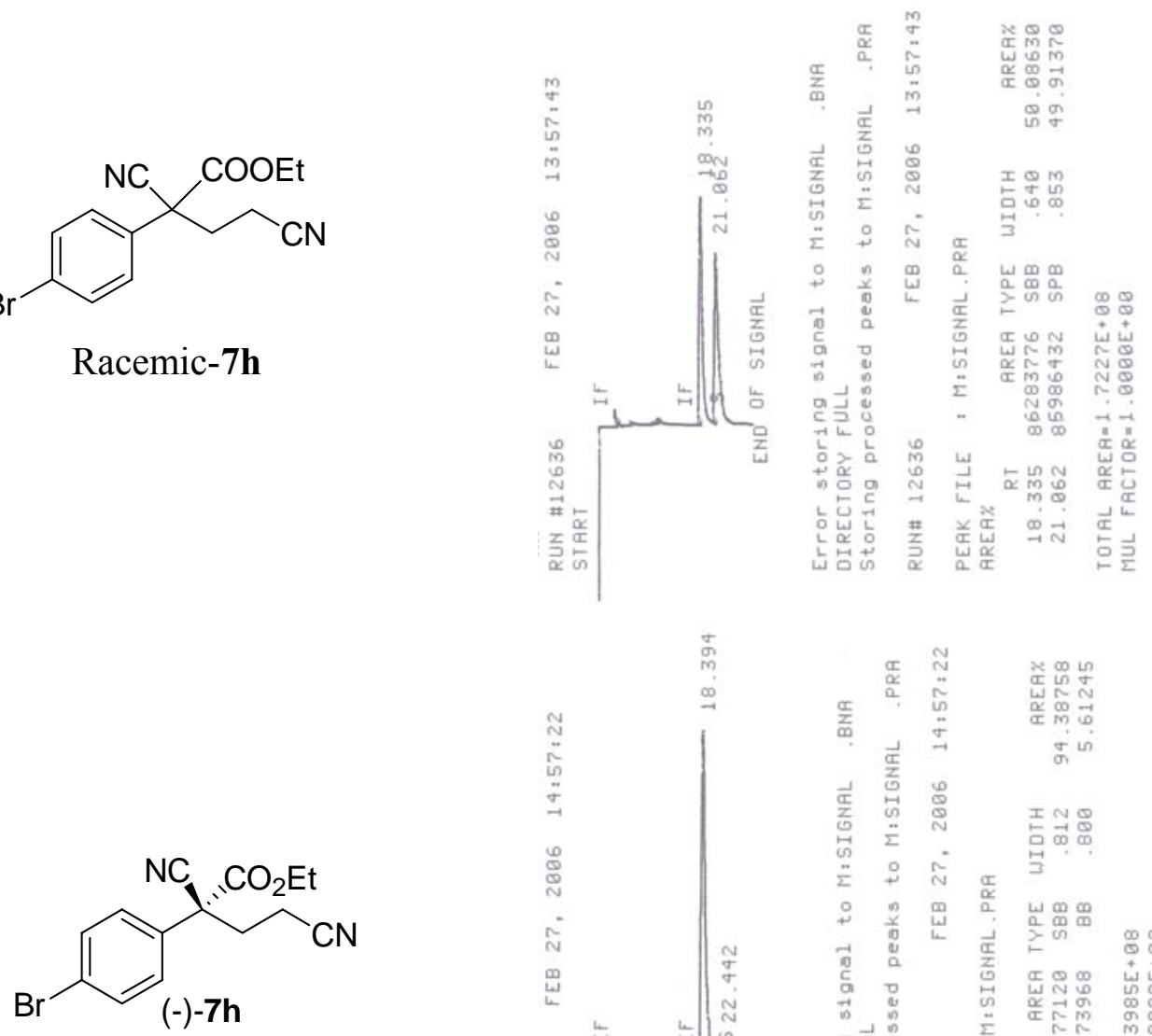

$89 \%$ ee, product obtained with Q-2c

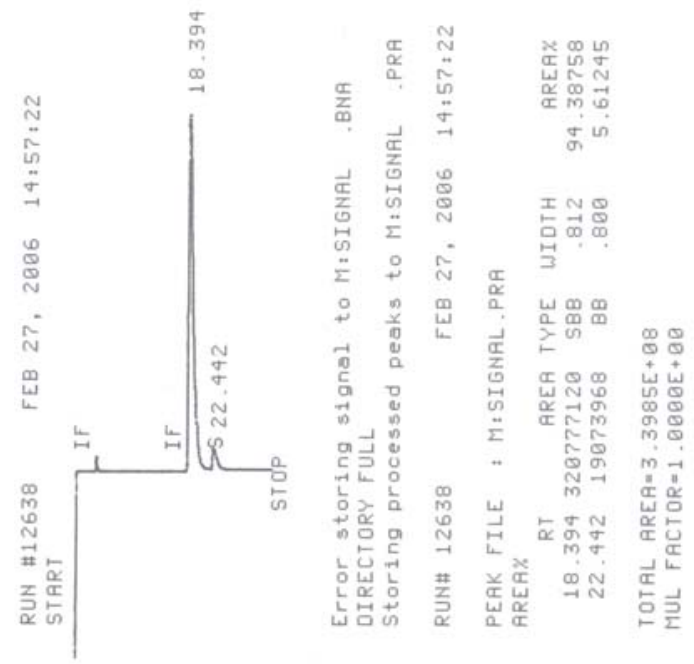<smiles>CCOC(=O)c1ccc(Br)cc1</smiles>

$89 \%$ ee, product obtained with QD-2c

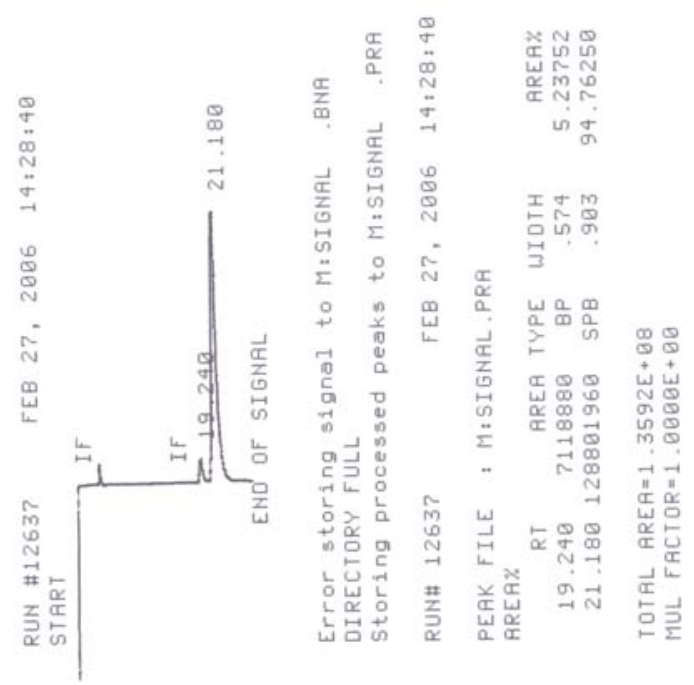


HPLC conditions for ee determination: Chiralcel OJ, hexanes: isopropanol $=60: 40,1.0$ $\mathrm{mL} / \mathrm{min}, 220 \mathrm{~nm}$.
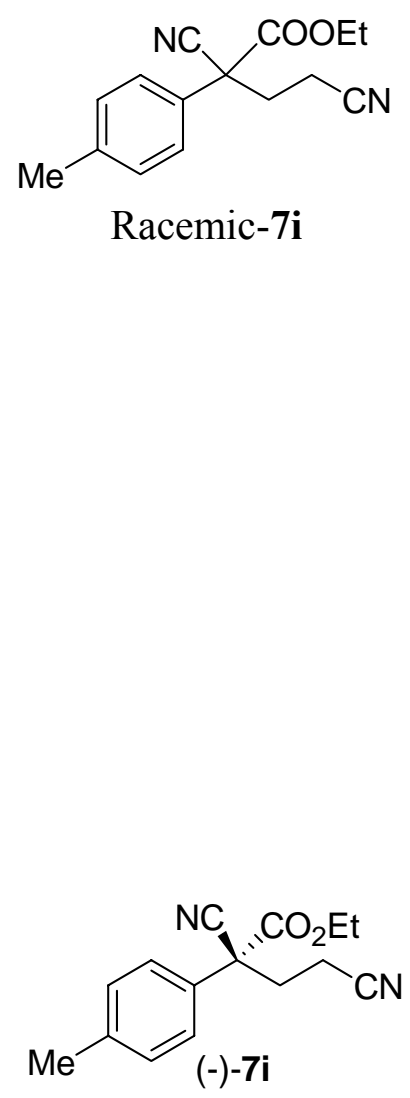

$90 \%$ ee, product obtained with Q-2c
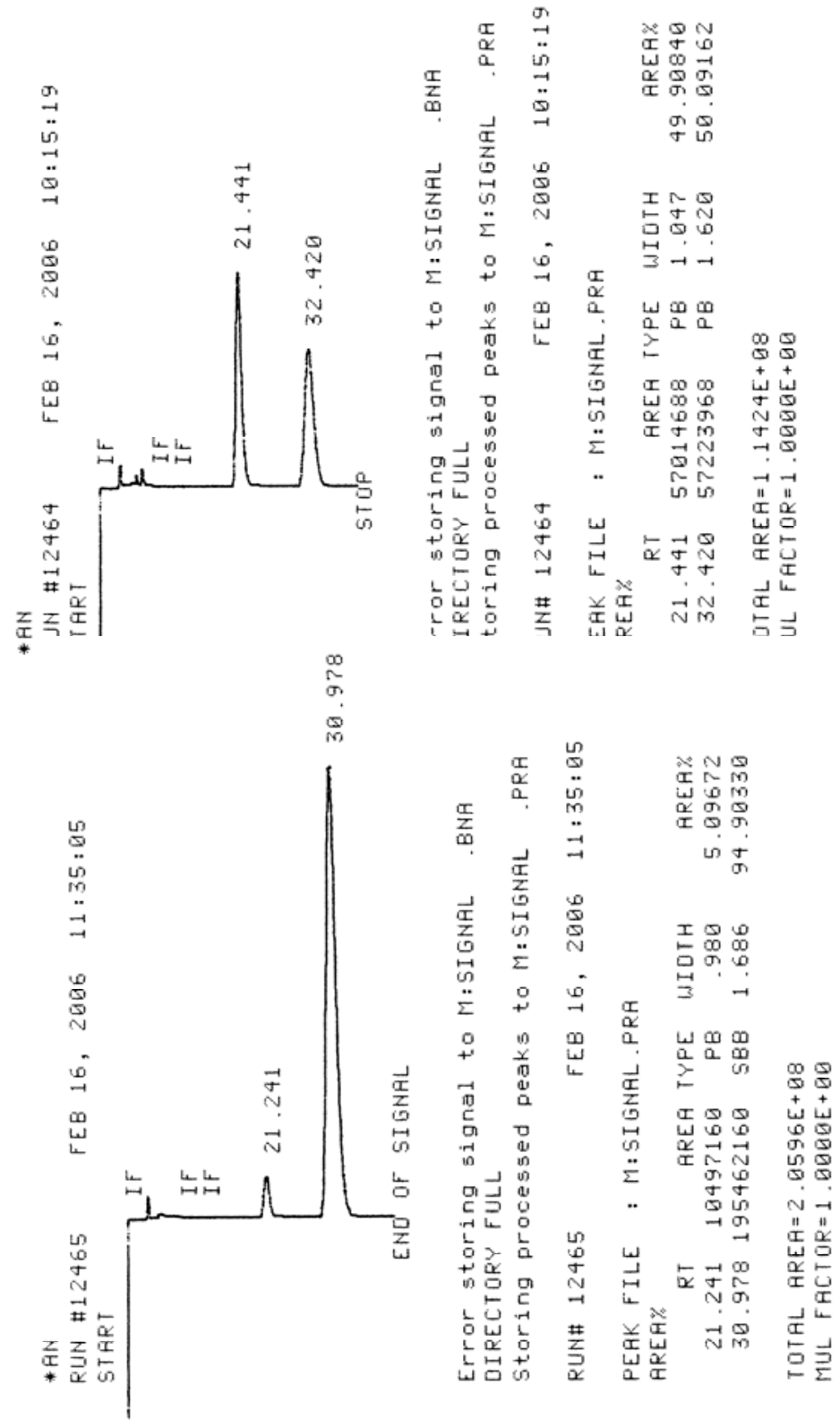
HPLC conditions for ee determination: Chiralcel OD, hexanes: isopropanol $=80: 20,1.0$ $\mathrm{mL} / \mathrm{min}, 220 \mathrm{~nm}$.
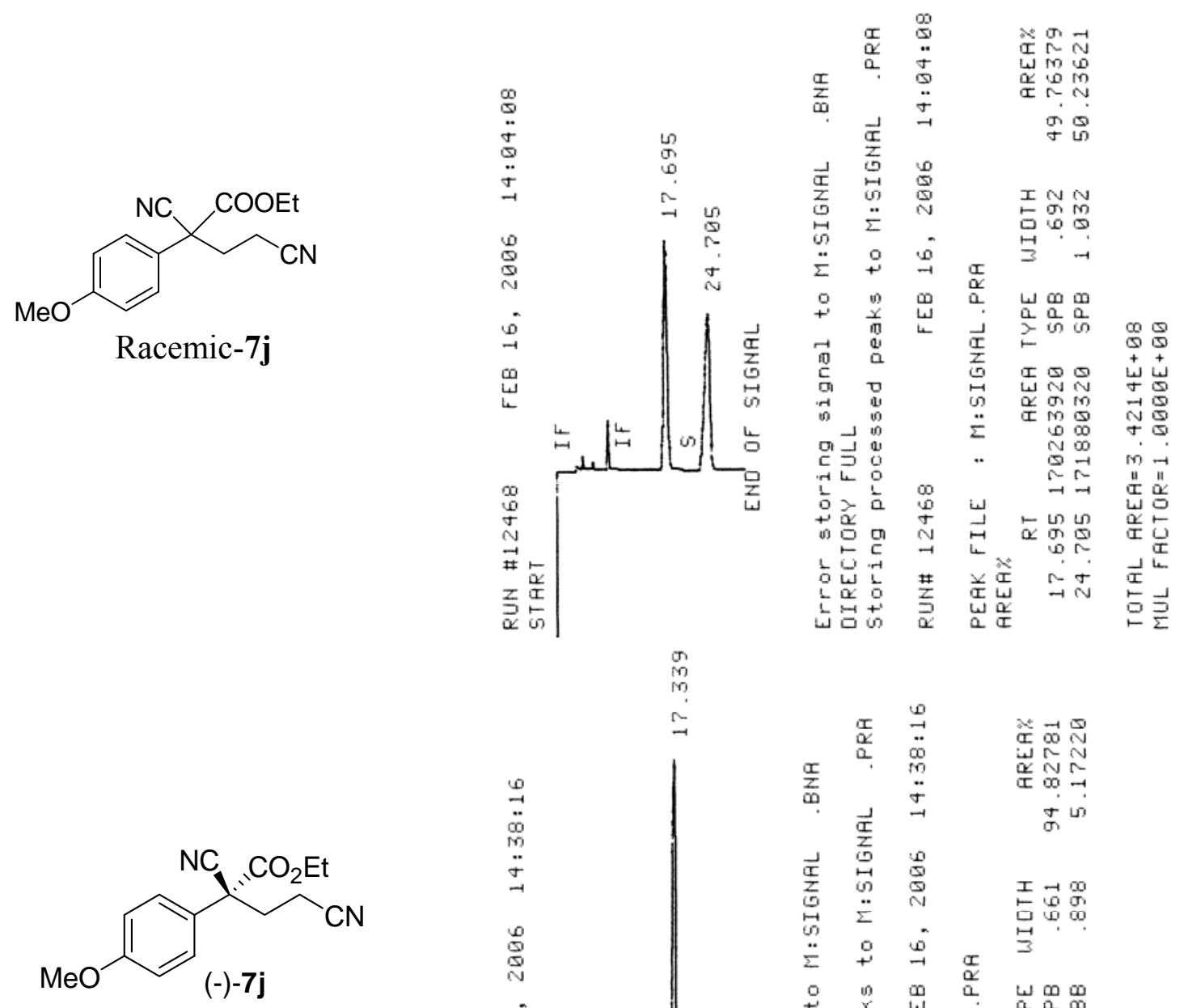

90\% ee, product obtained with Q-2c

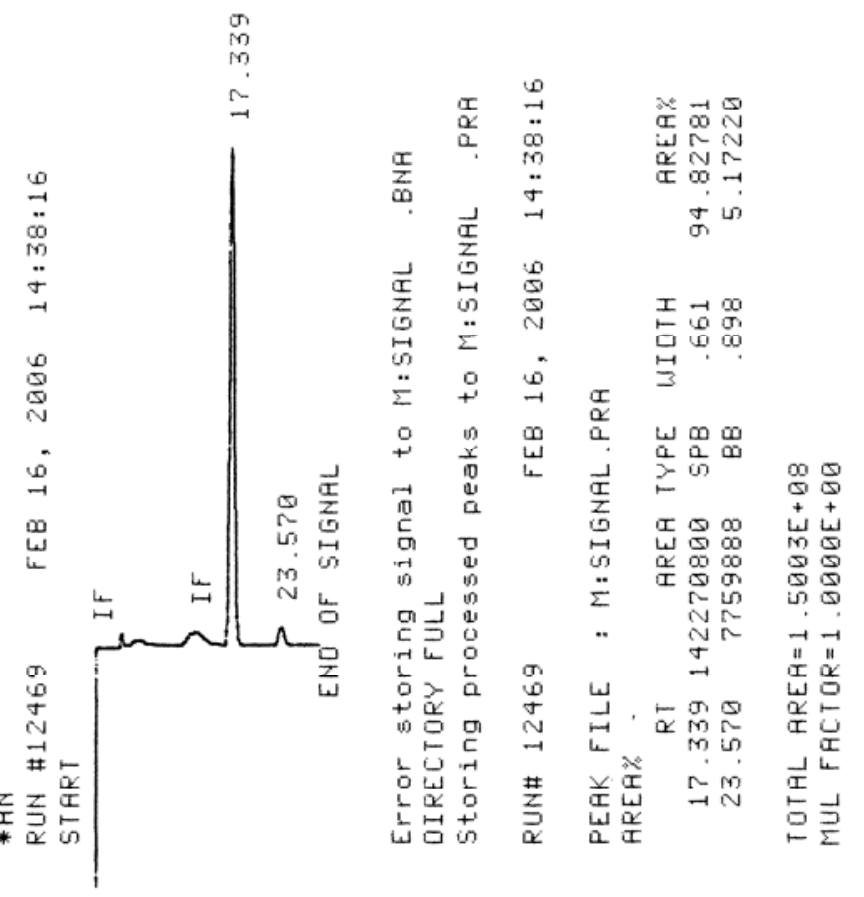


HPLC conditions for ee determination: $(\mathrm{R}, \mathrm{R})$-whelk-O 1, hexanes: isopropanol = 80: 20, $1.0 \mathrm{~mL} / \mathrm{min}, 280 \mathrm{~nm}$.

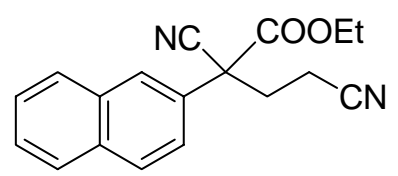

Racemic-7k

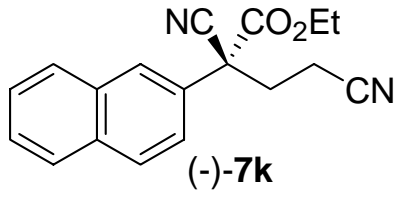

$89 \%$ ee, product obtained with Q-2c
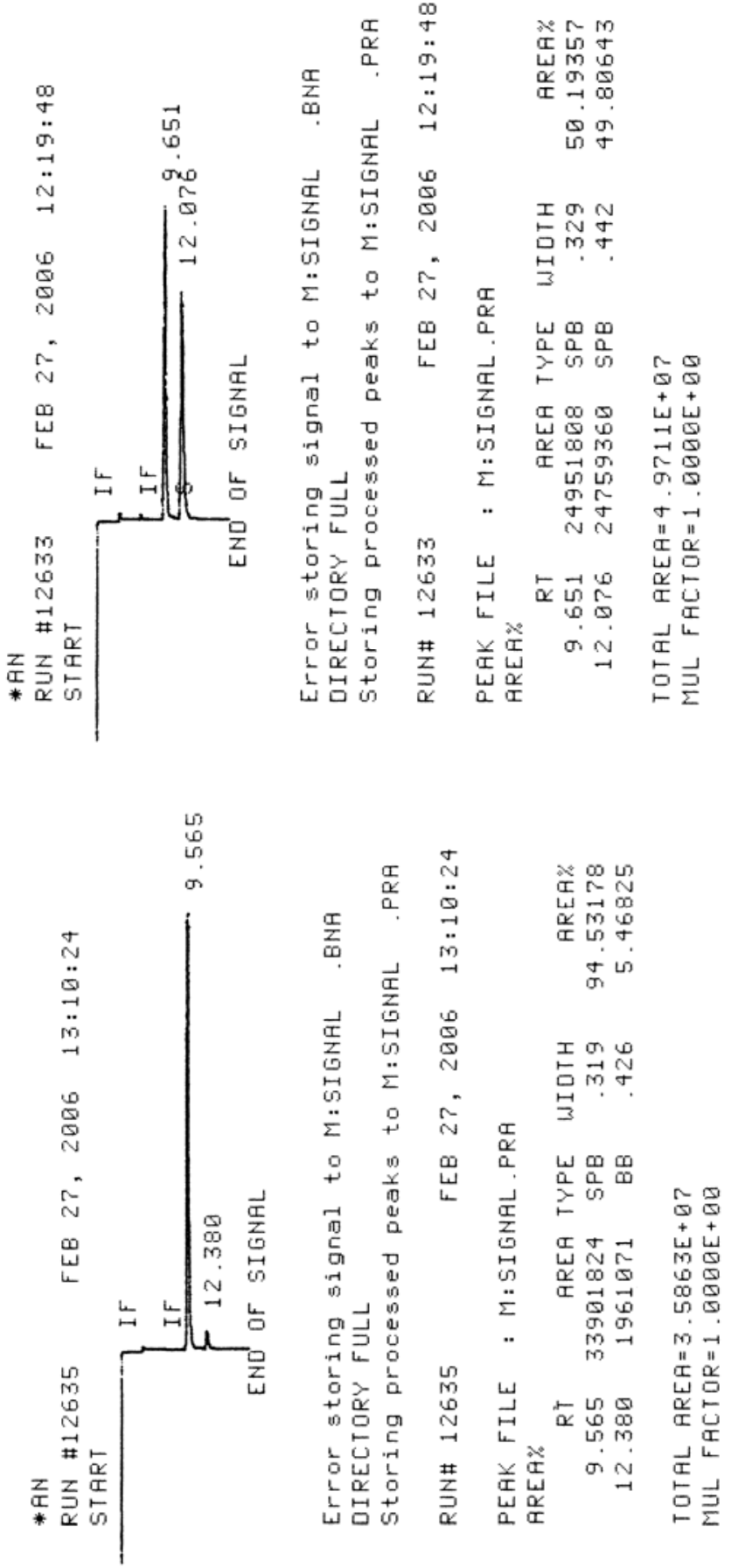
HPLC conditions for ee determination: Chiralpak AS, hexanes: isopropanol $=80: 20,1.0$ $\mathrm{mL} / \mathrm{min}, 220 \mathrm{~nm}$.

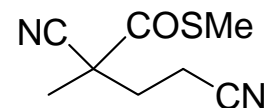

Racemic-7l

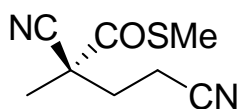

(-)-7I

$93 \%$ ee, product obtained with Q-2c
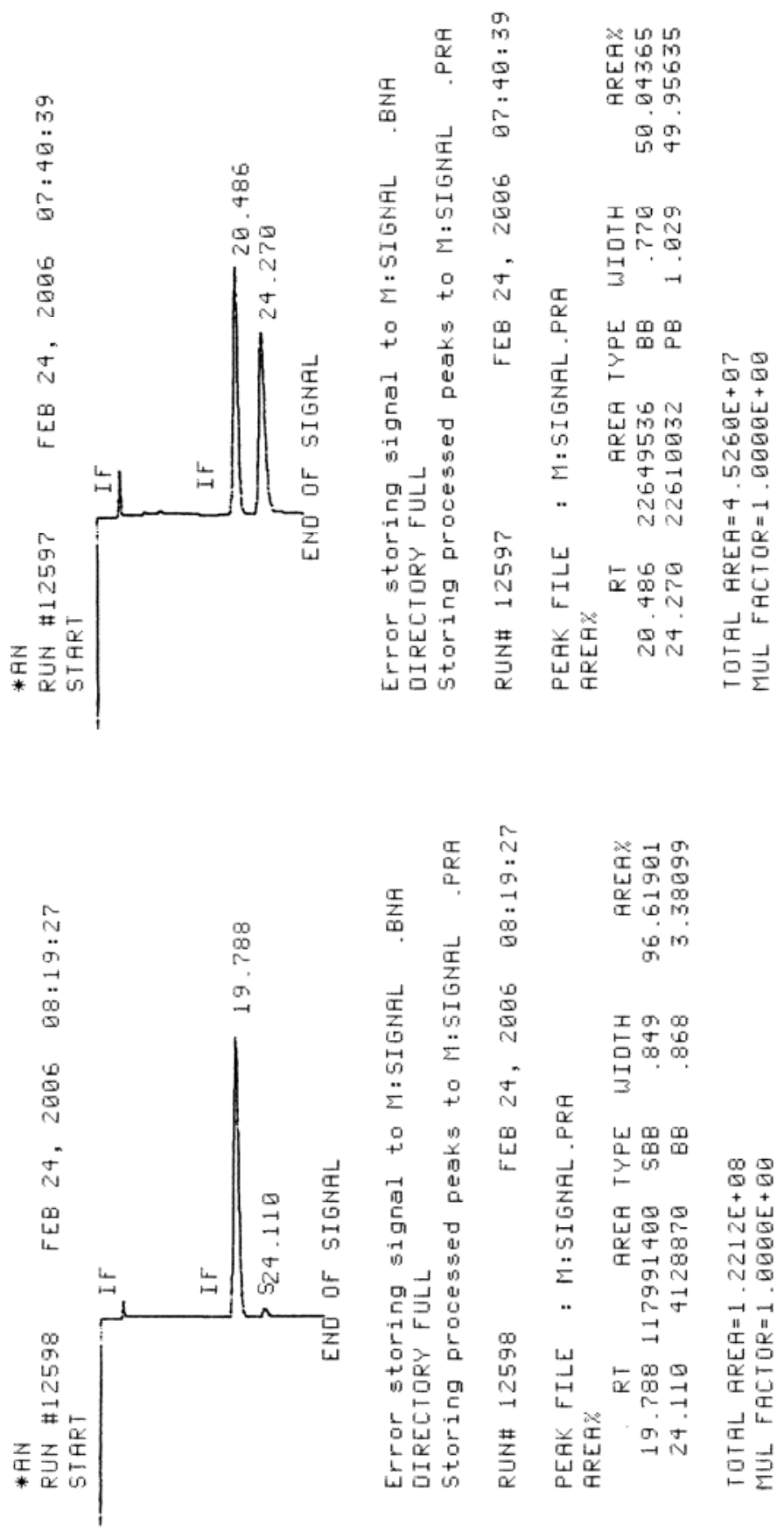
HPLC conditions for ee determination: Chiralpak AS, hexanes: isopropanol $=85: 15,1.0$ $\mathrm{mL} / \mathrm{min}, 220 \mathrm{~nm}$.
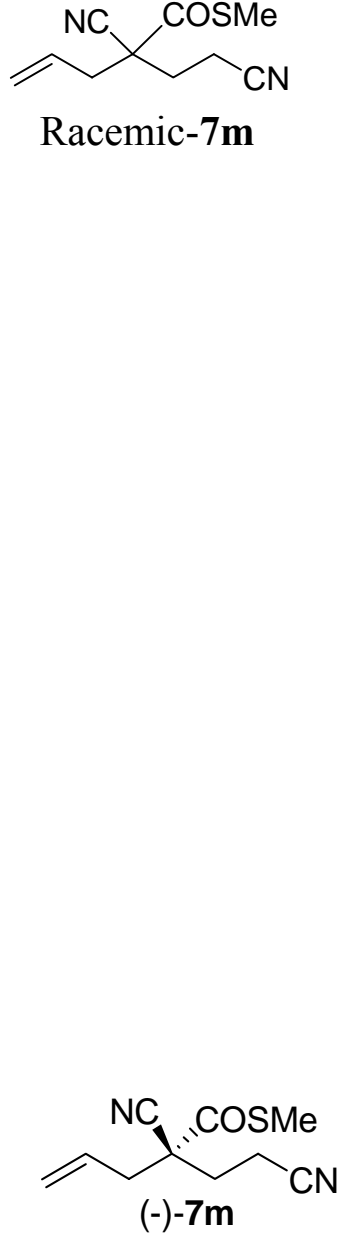

$91 \%$ ee, product obtained with Q-2c
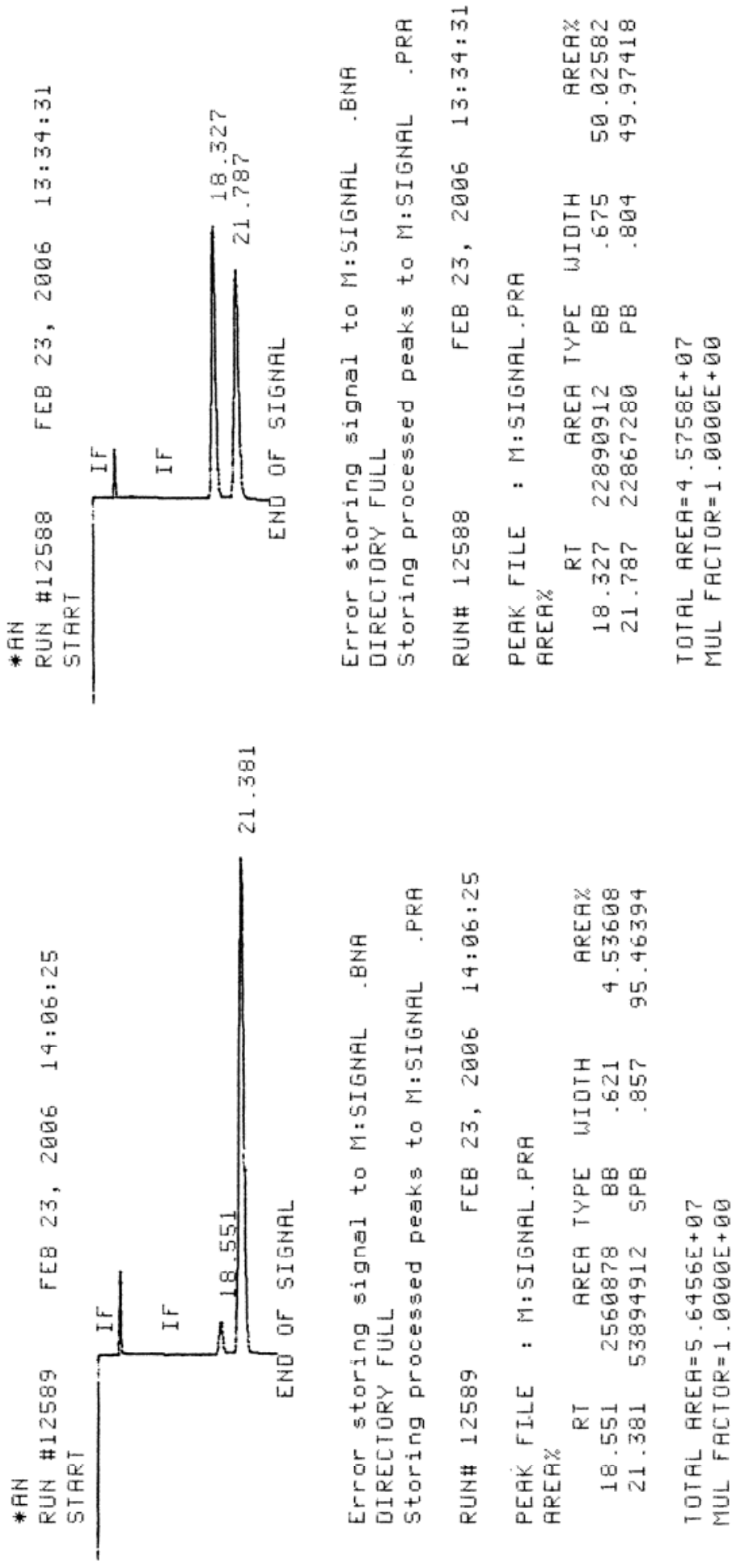


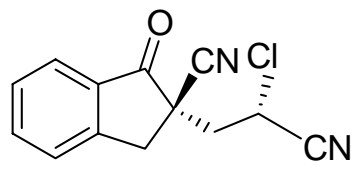

$5 a$
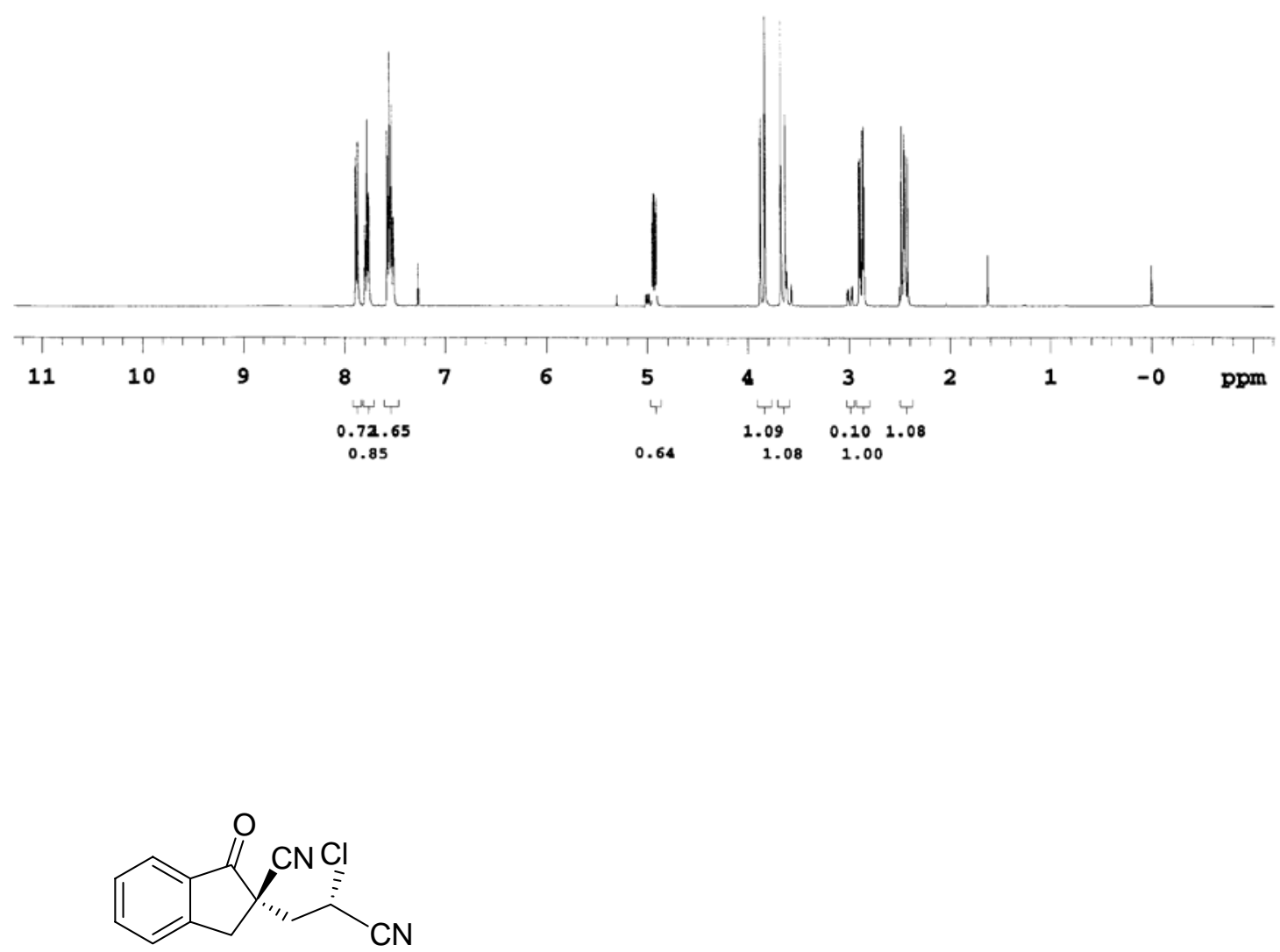

$5 a$

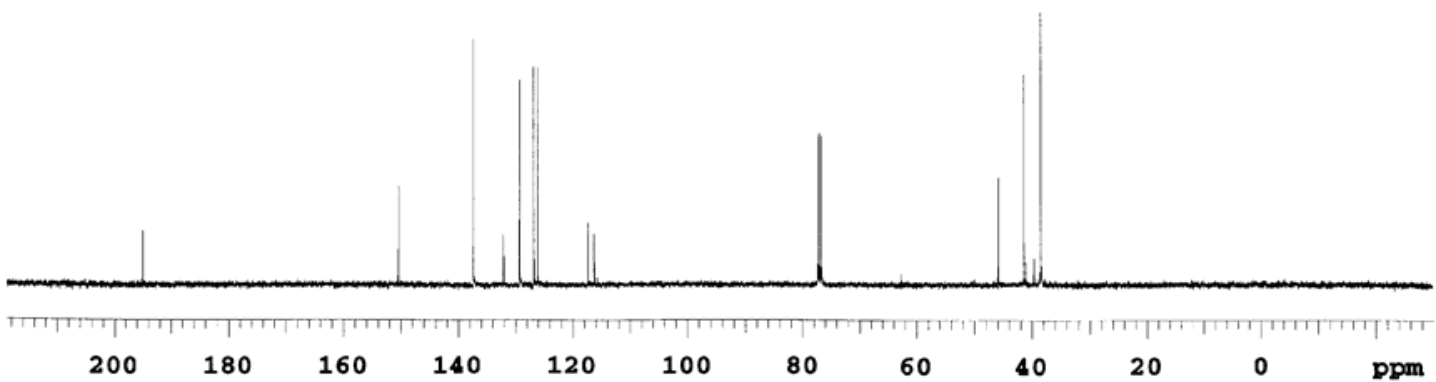



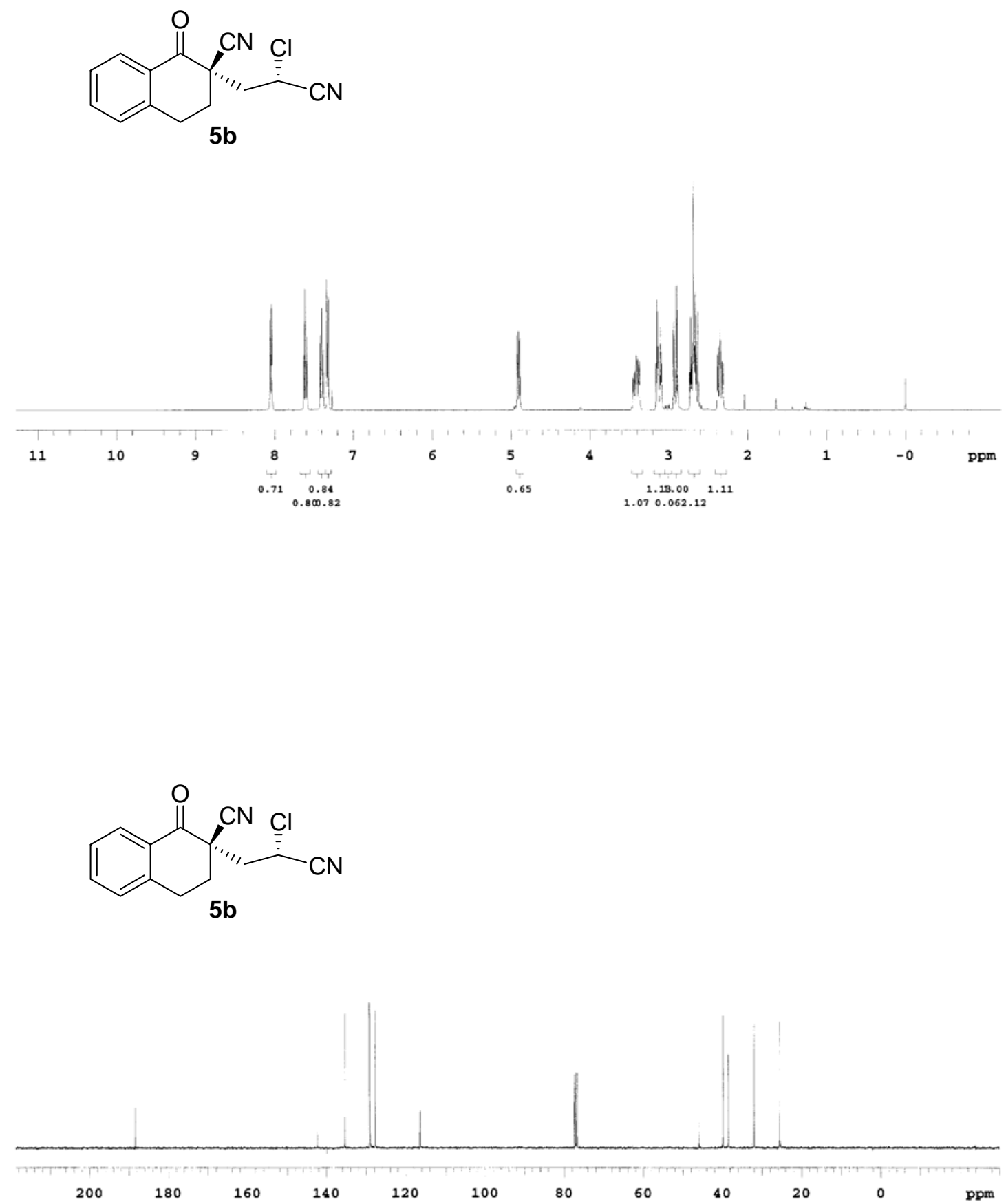

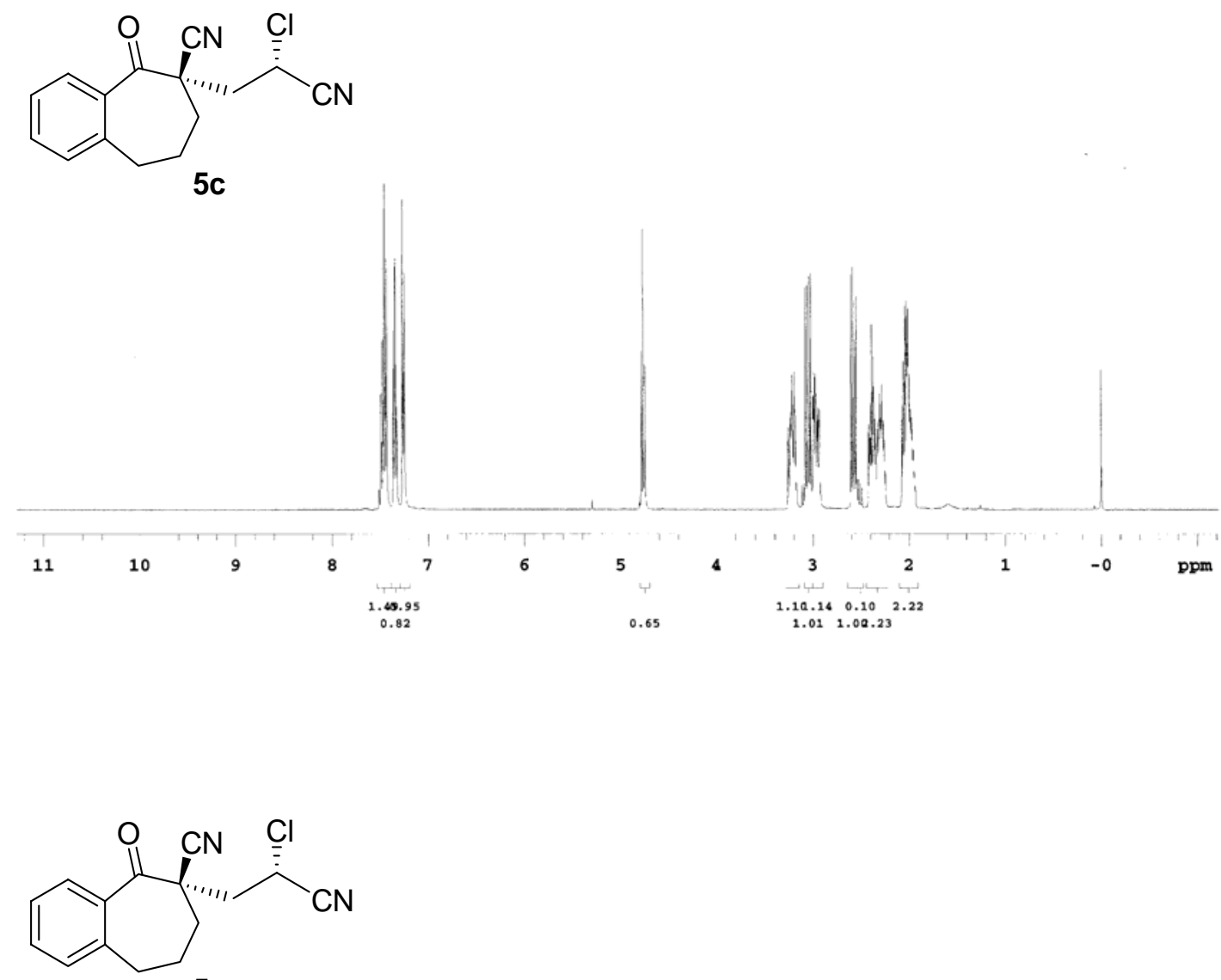

$5 c$

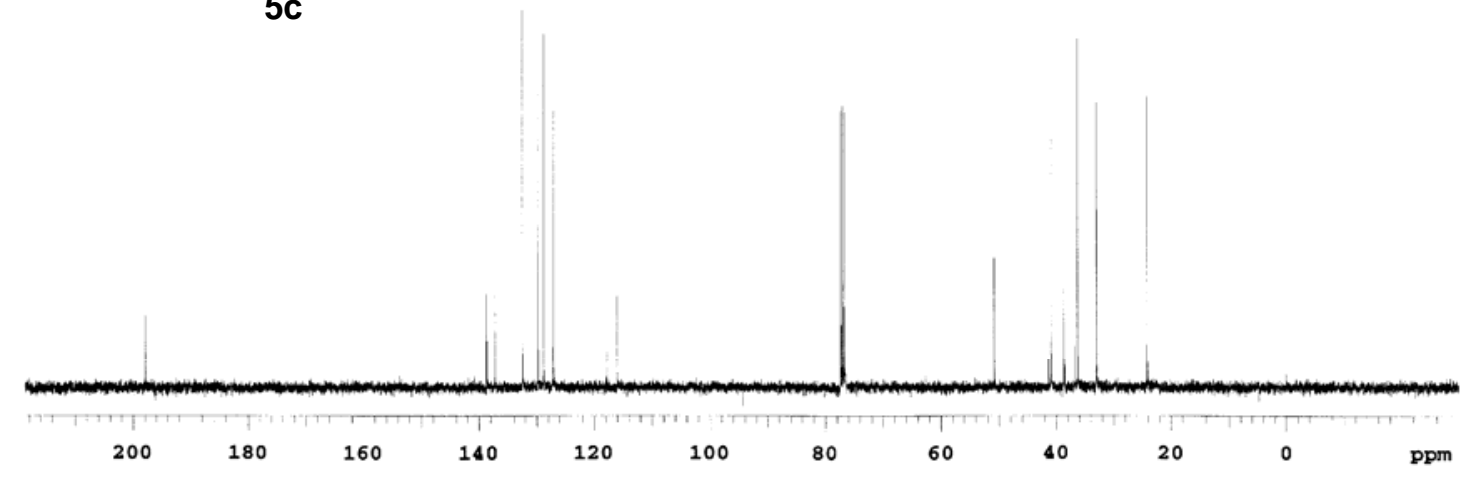



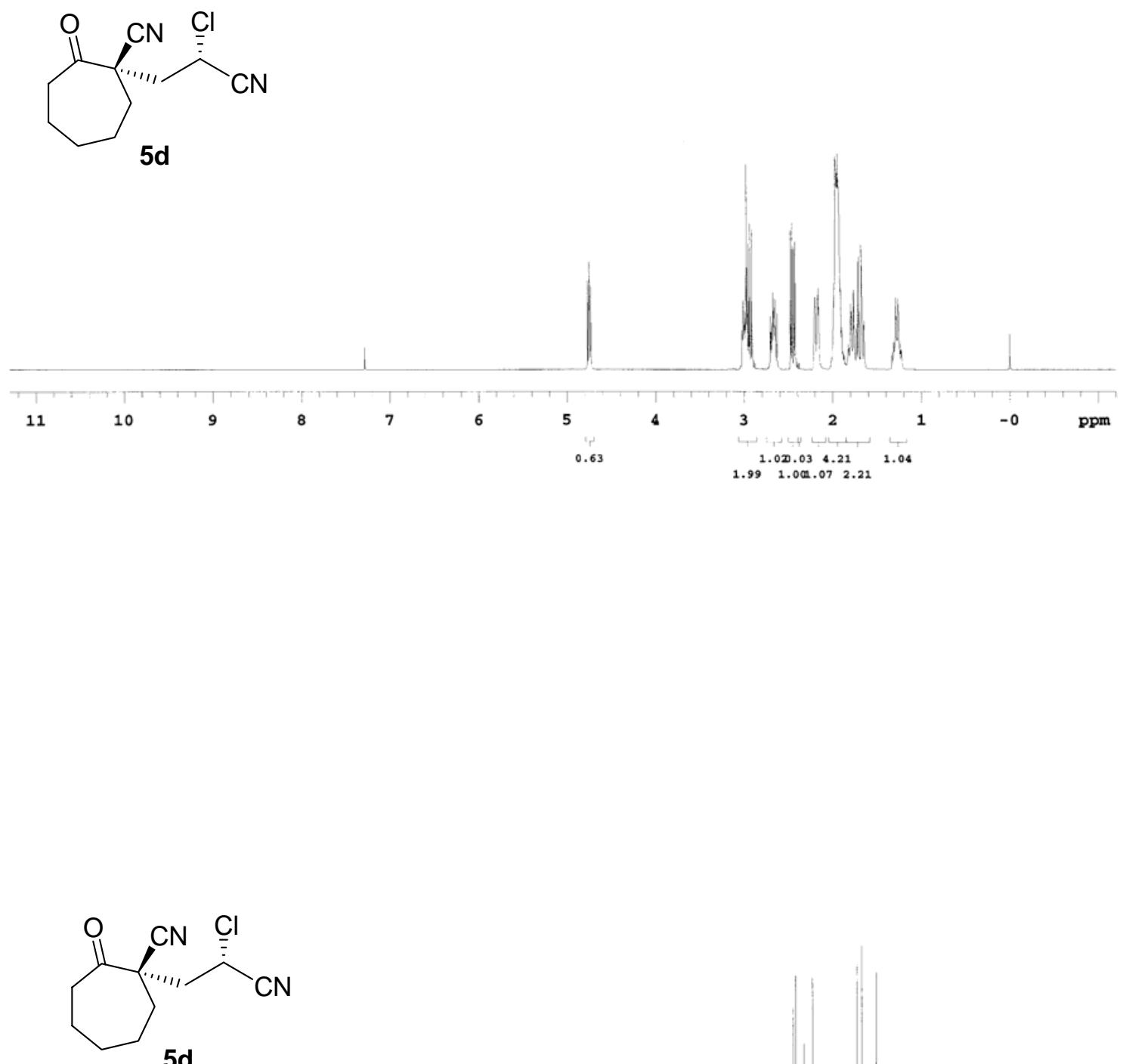

5d

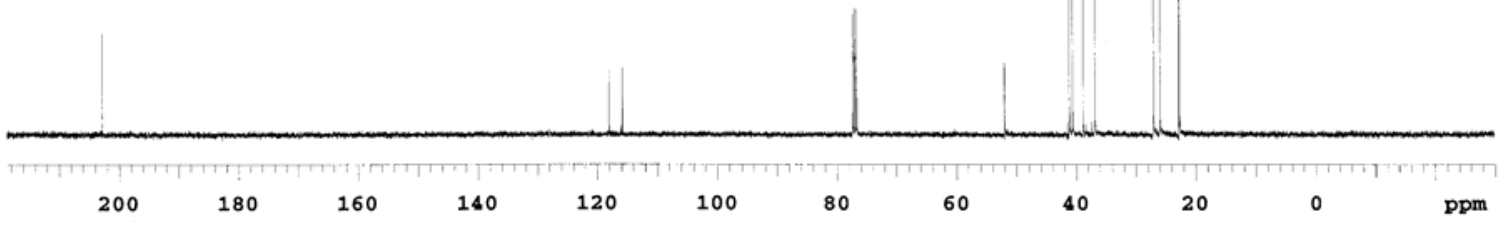



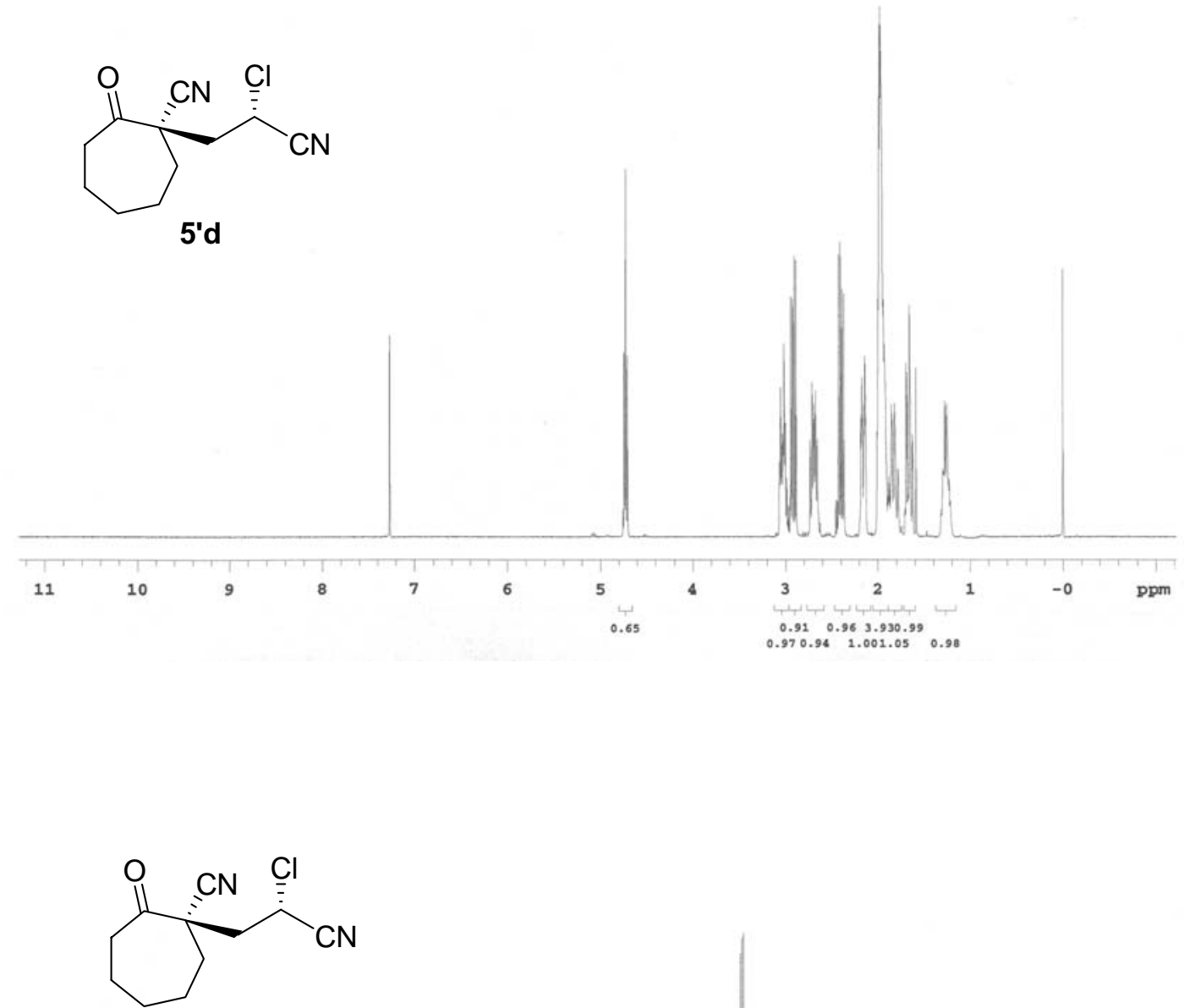

5'd

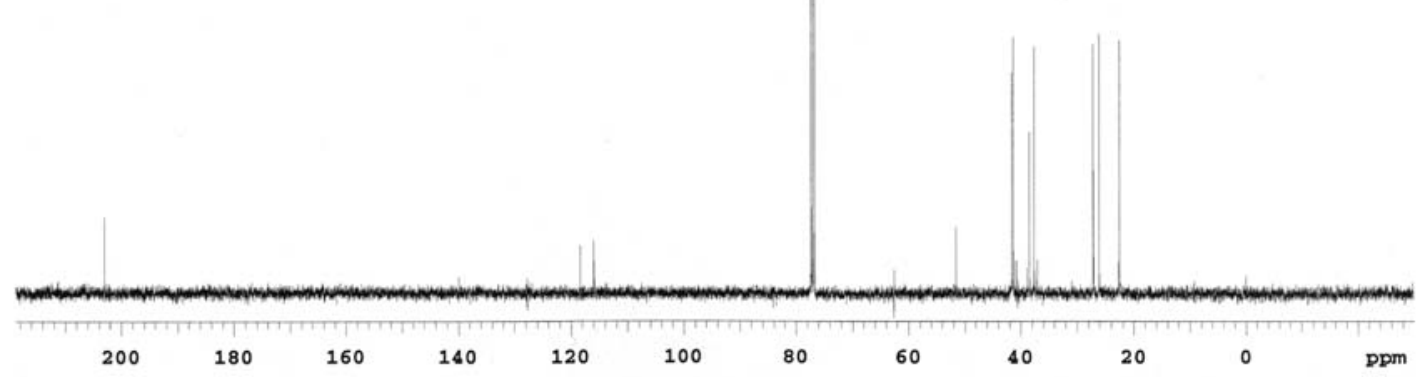



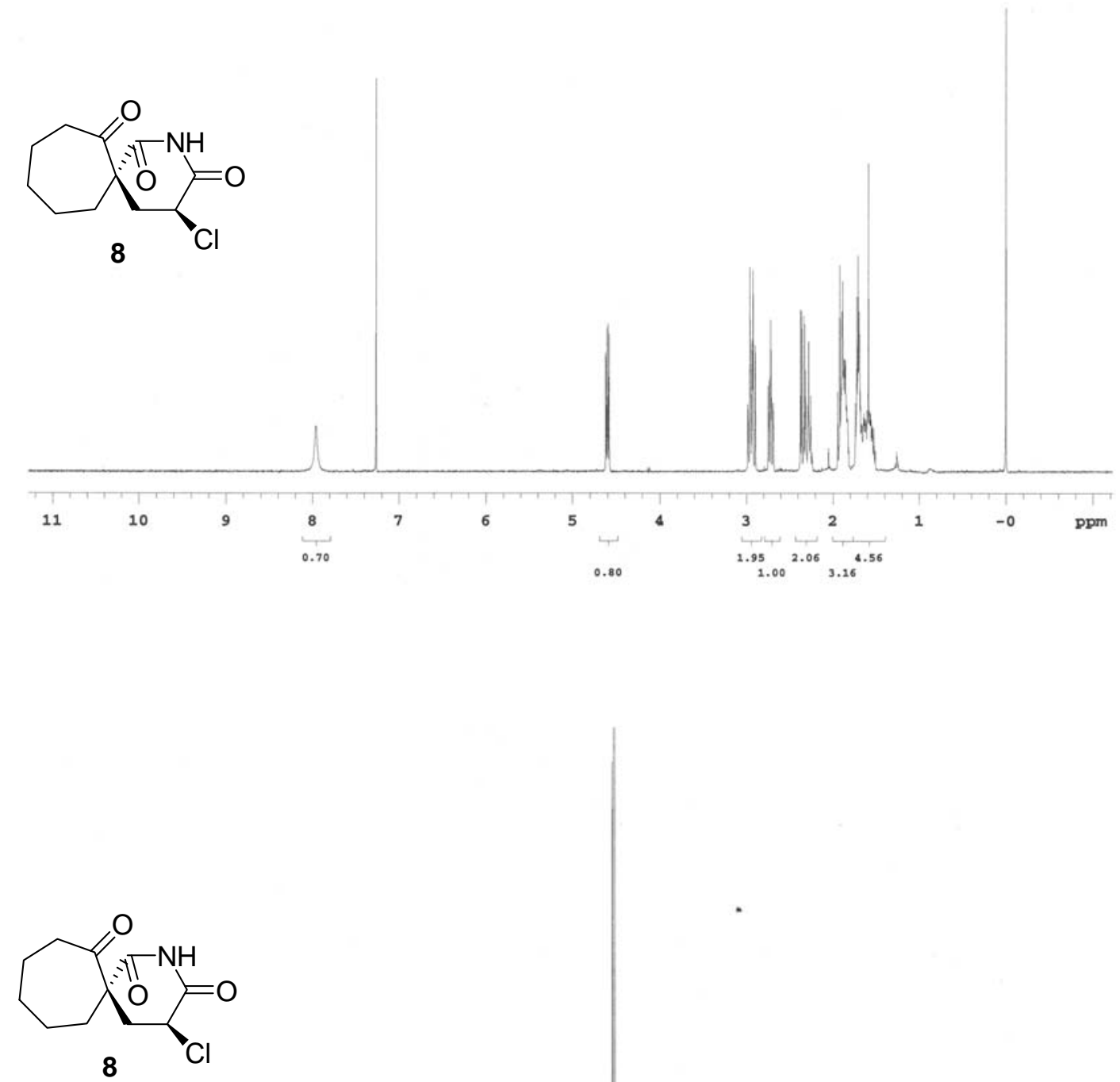

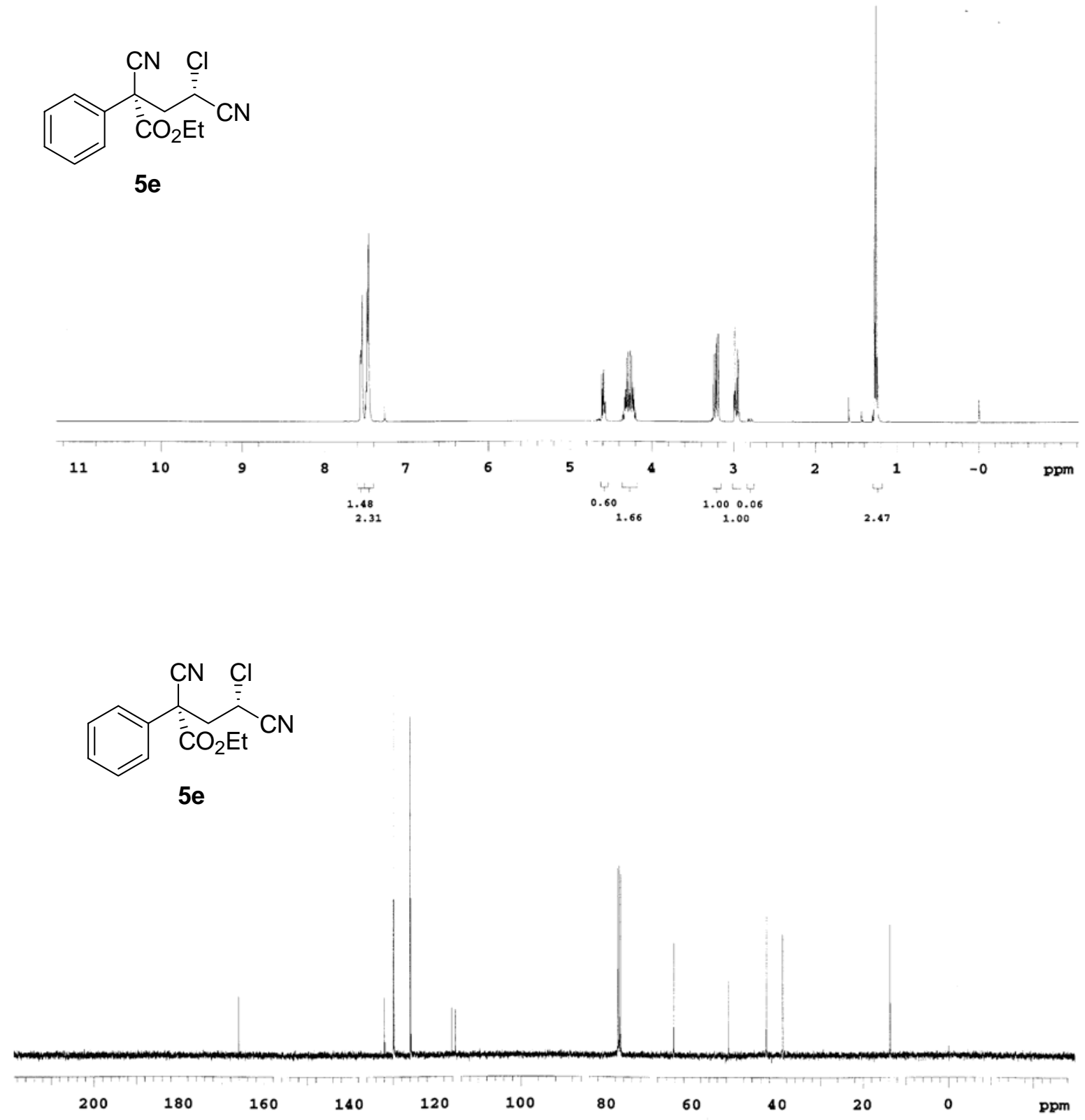

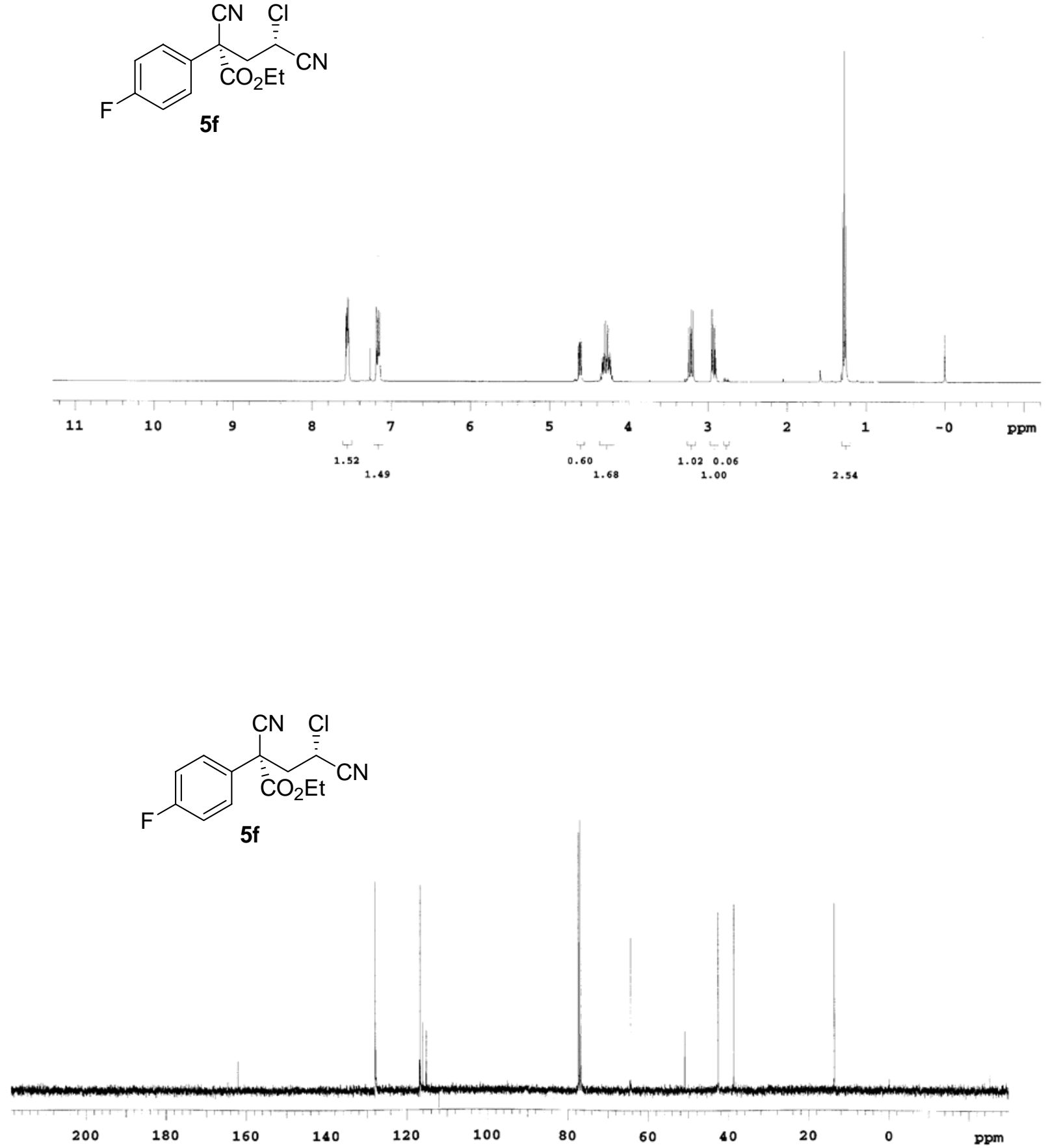

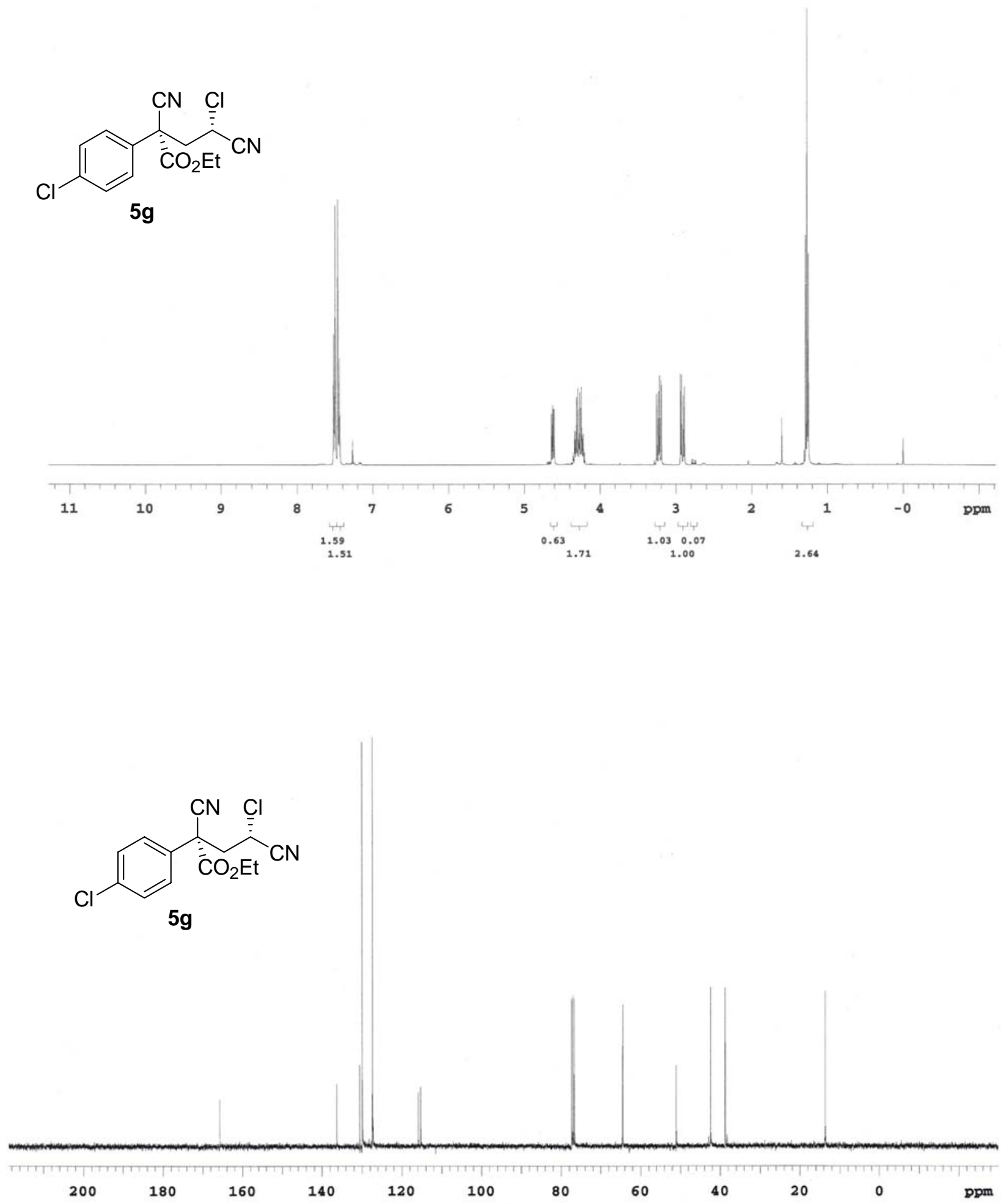


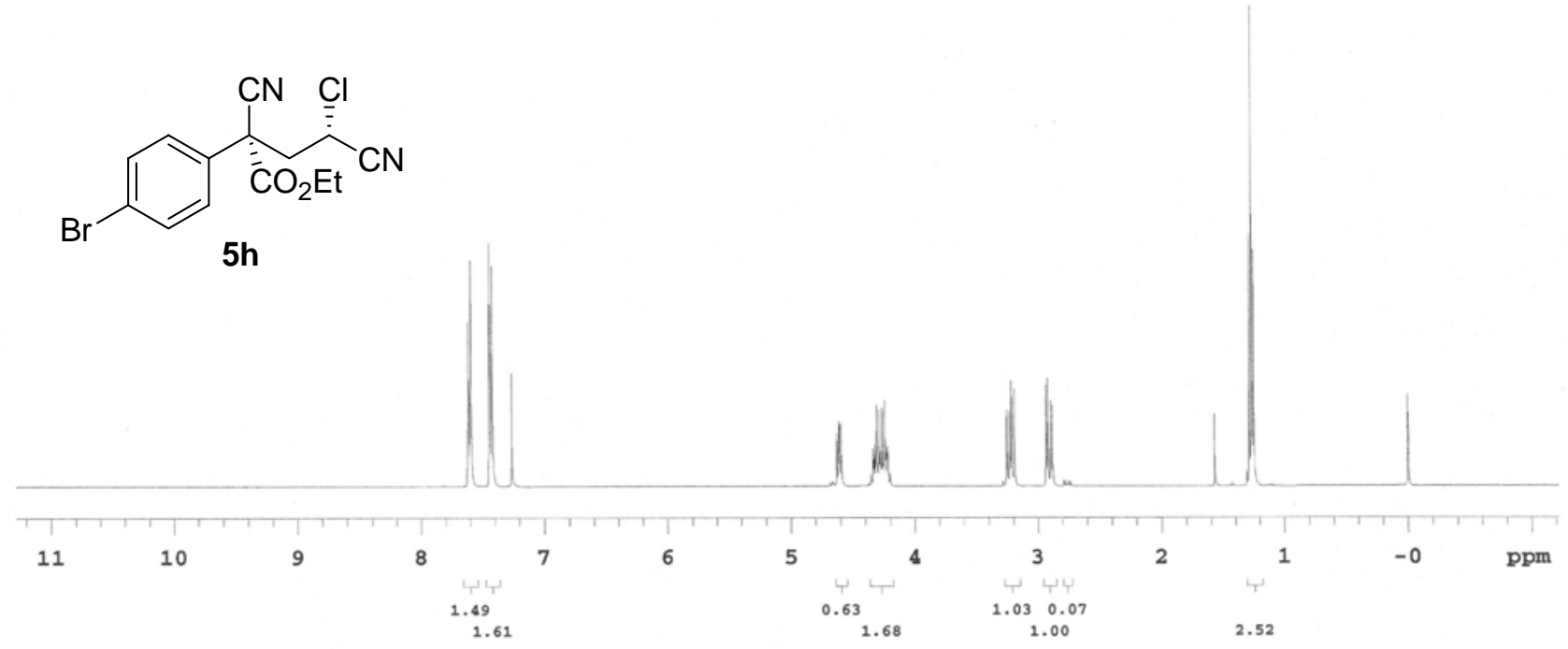

$5 h$ 

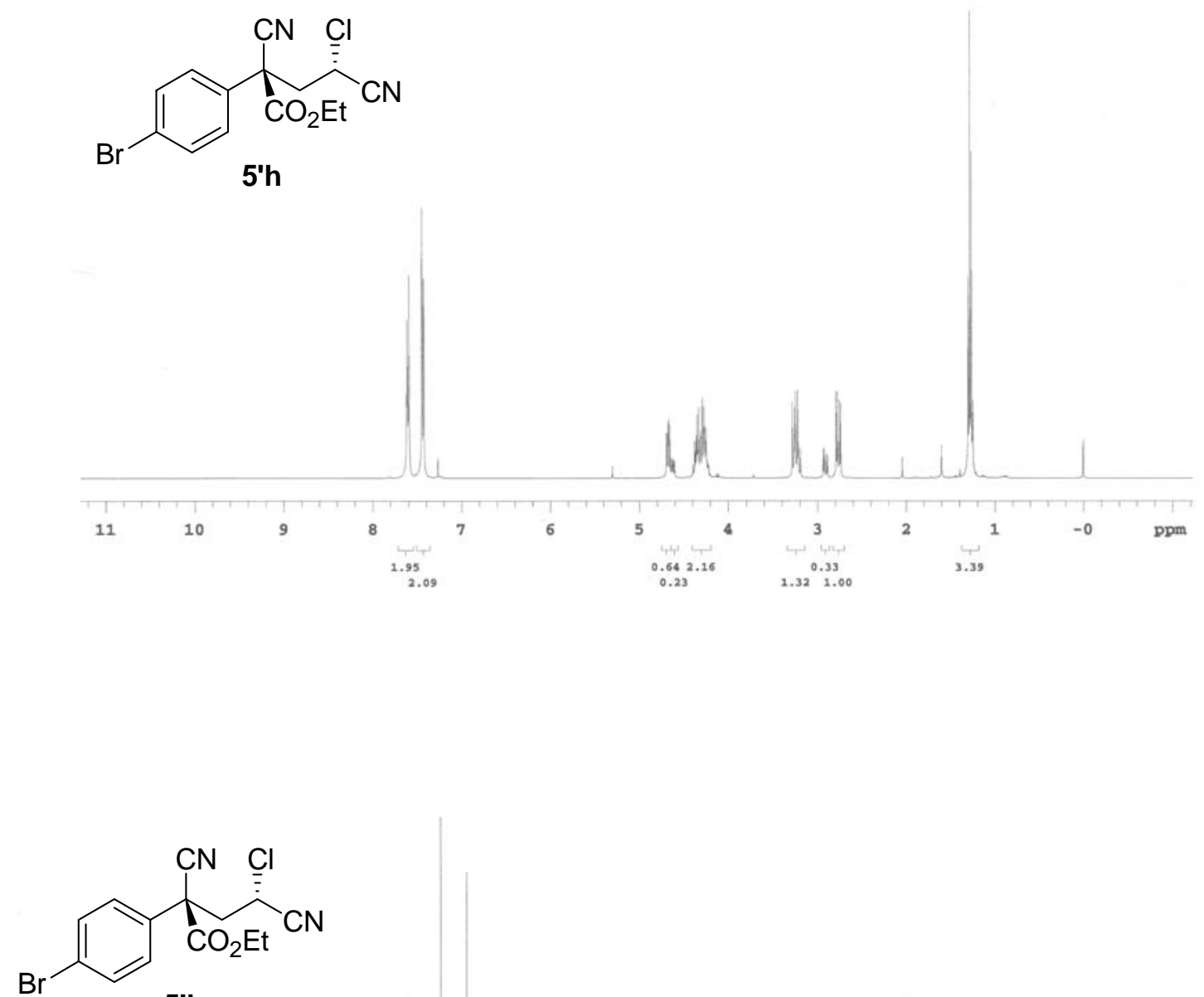

5'h 
<smiles>CCOC(=O)C(C#N)(CC(Cl)Cl)c1ccc(C)cc1</smiles>

$5 i$
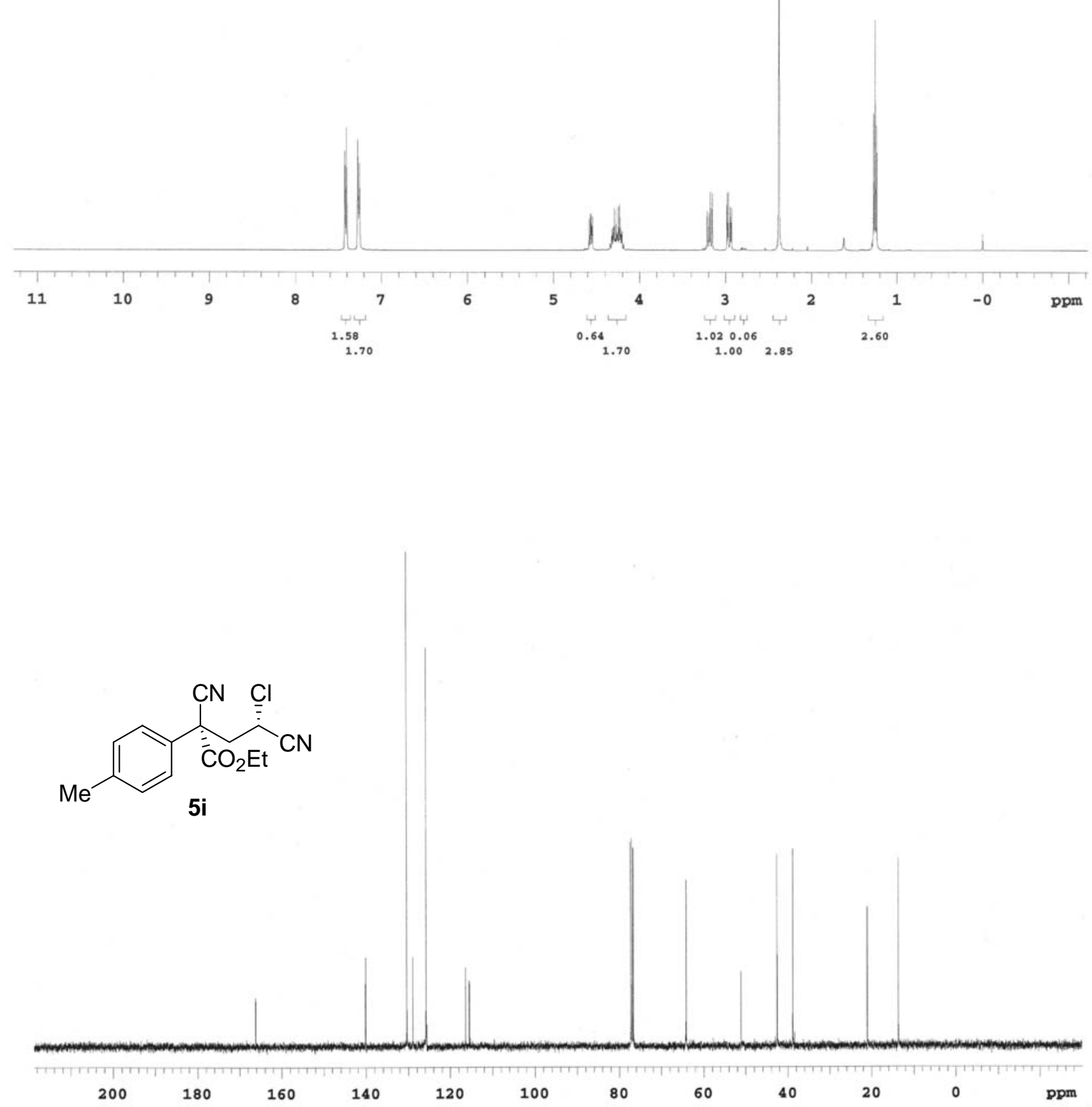

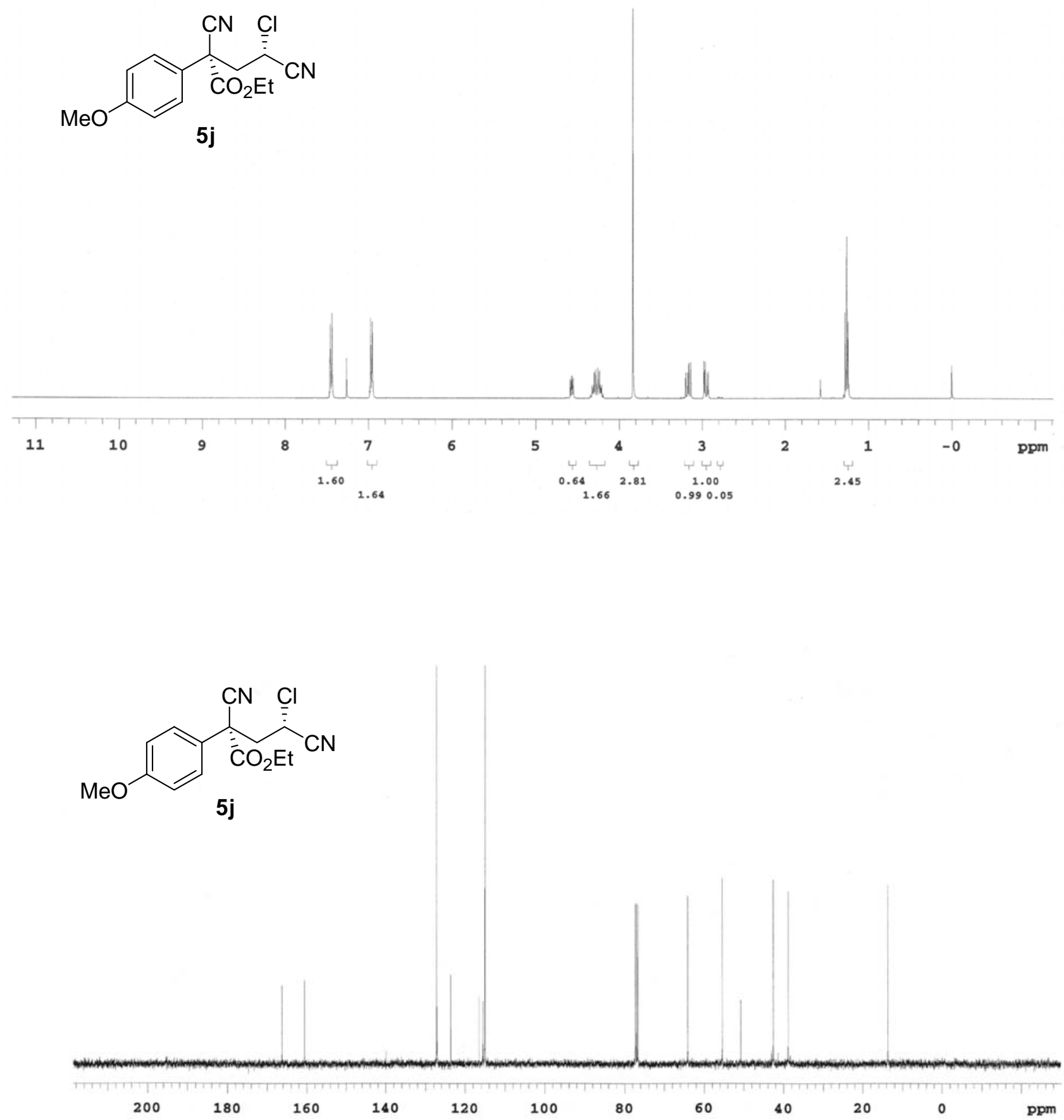
<smiles>CCOC(=O)C(C#N)(CC(Cl)C#N)c1ccc2ccccc2c1</smiles>

$5 k$

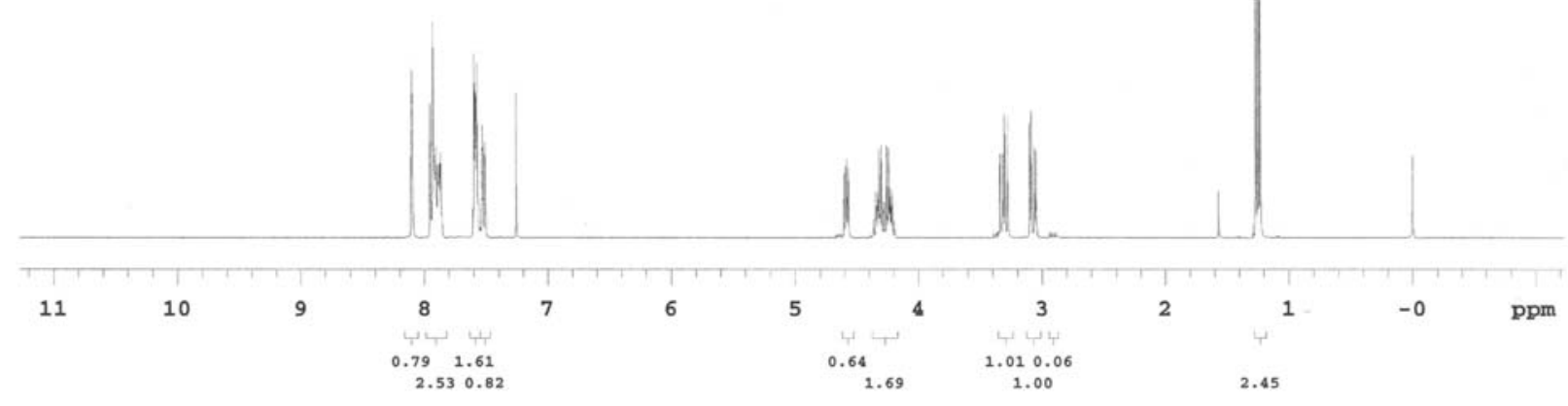<smiles>CCOC(=O)C(C#N)(c1ccc2ccccc2c1)[C@@H](Cl)C[Mg]</smiles>

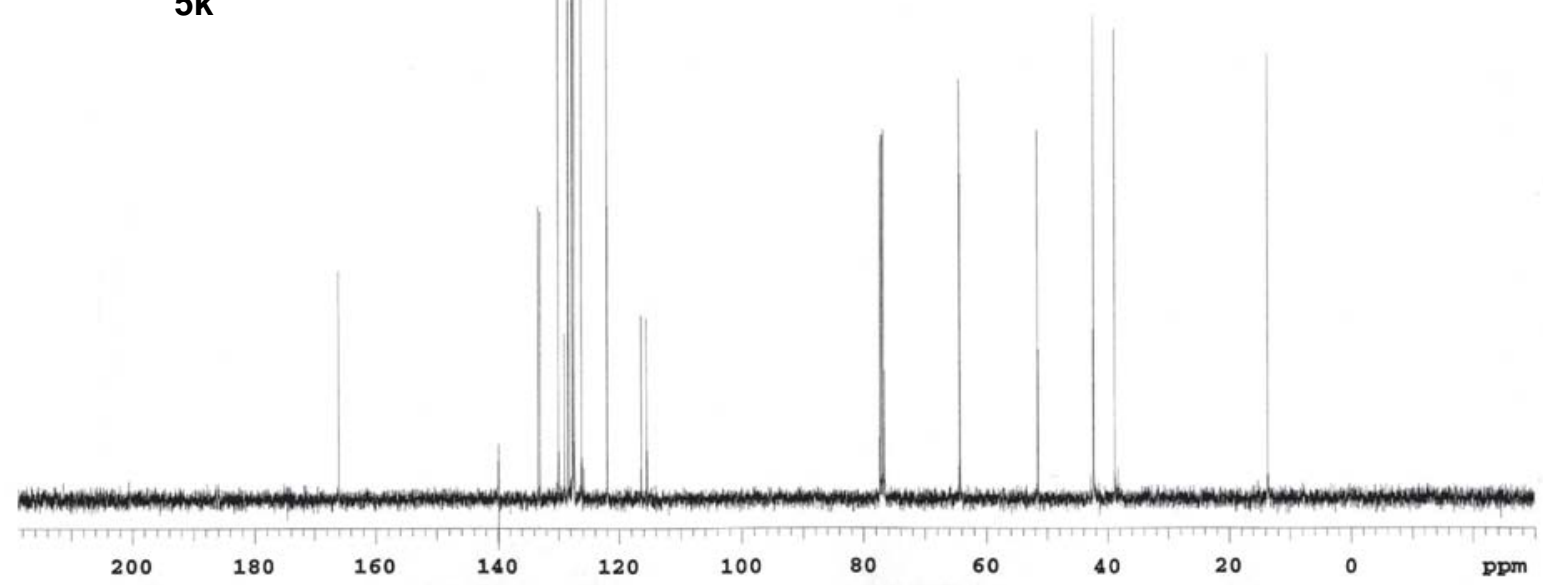



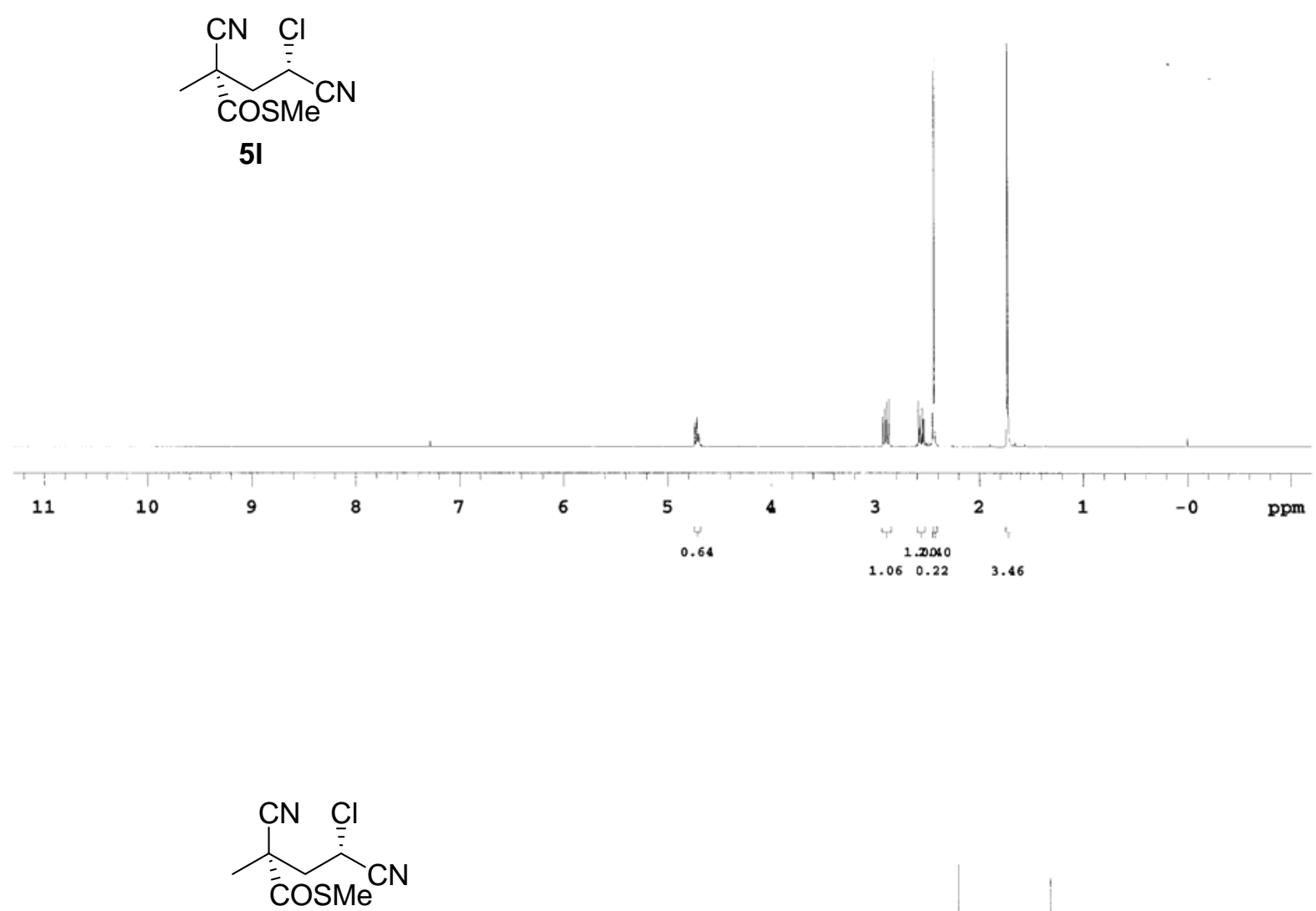

5

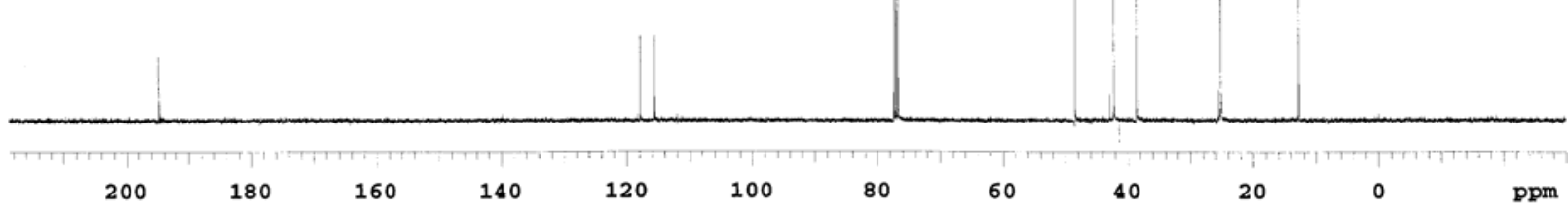



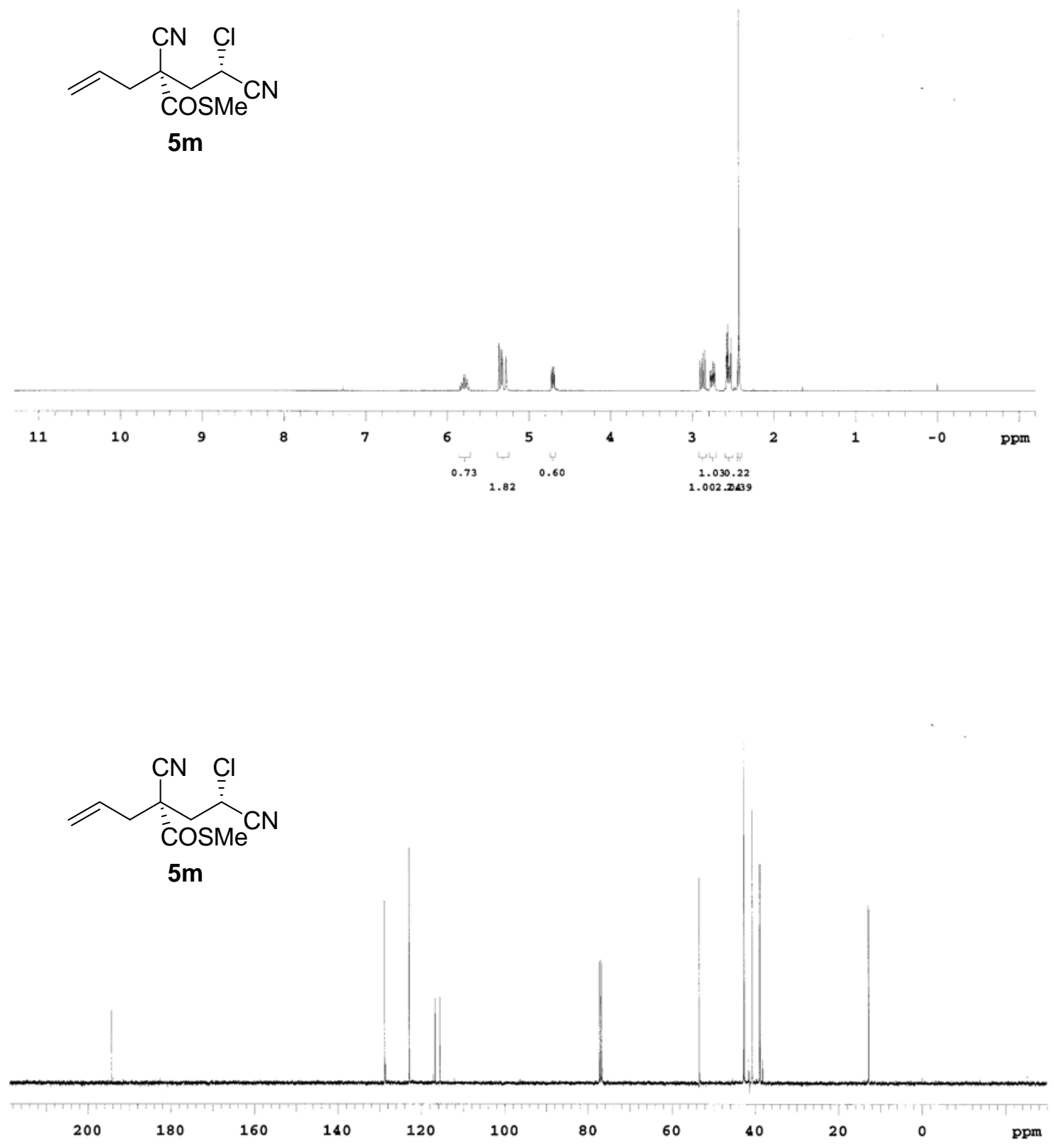

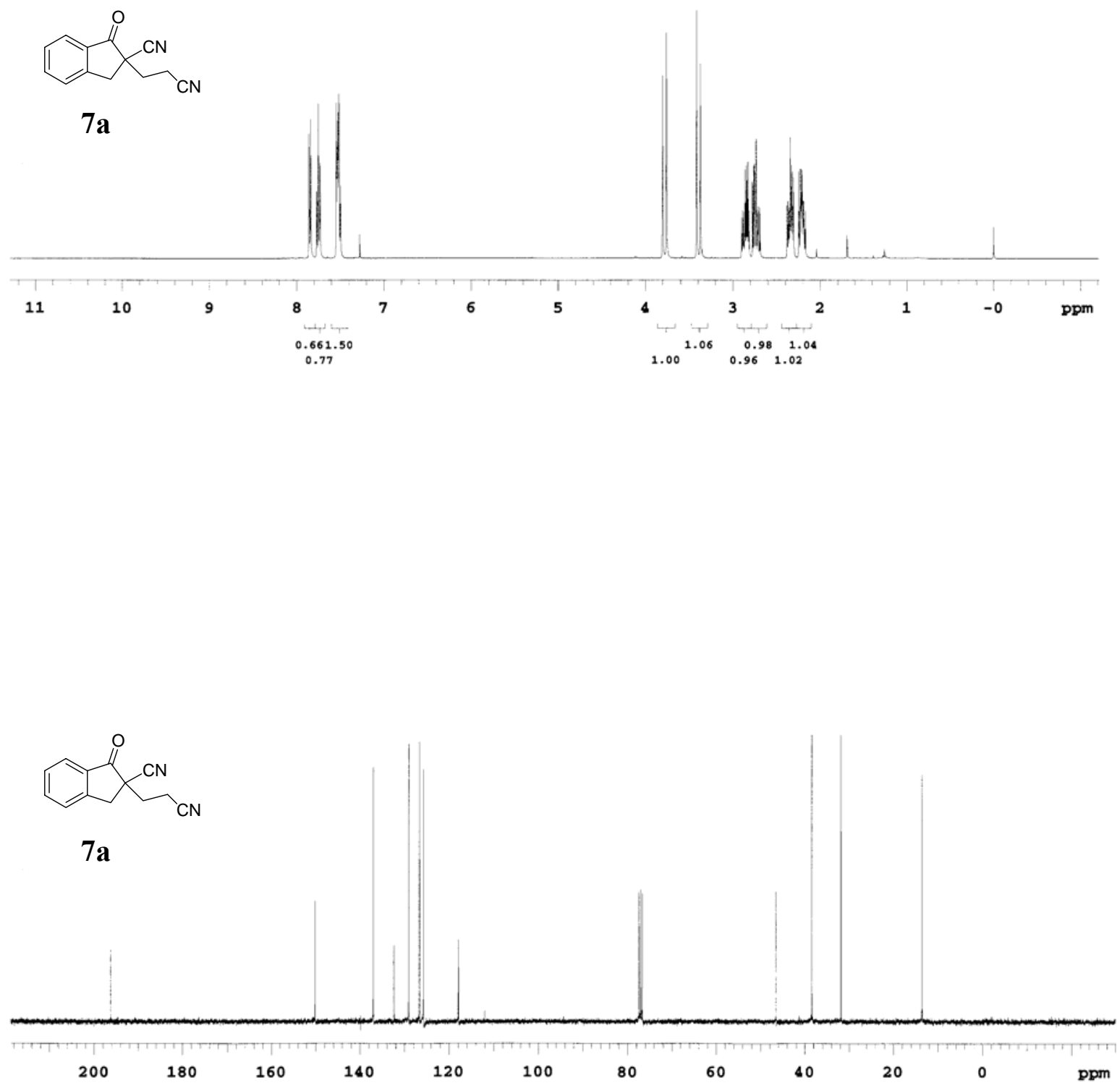

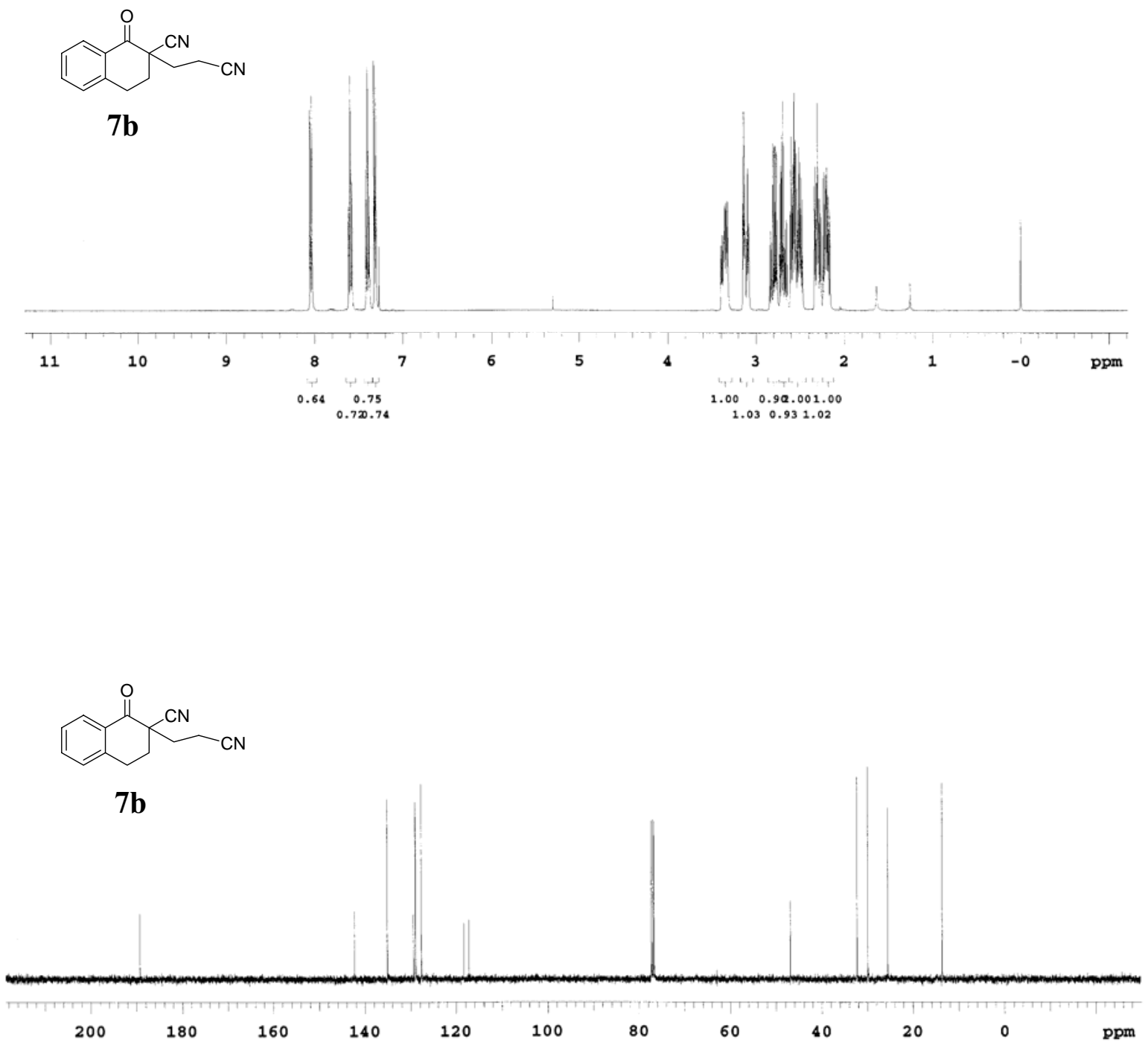

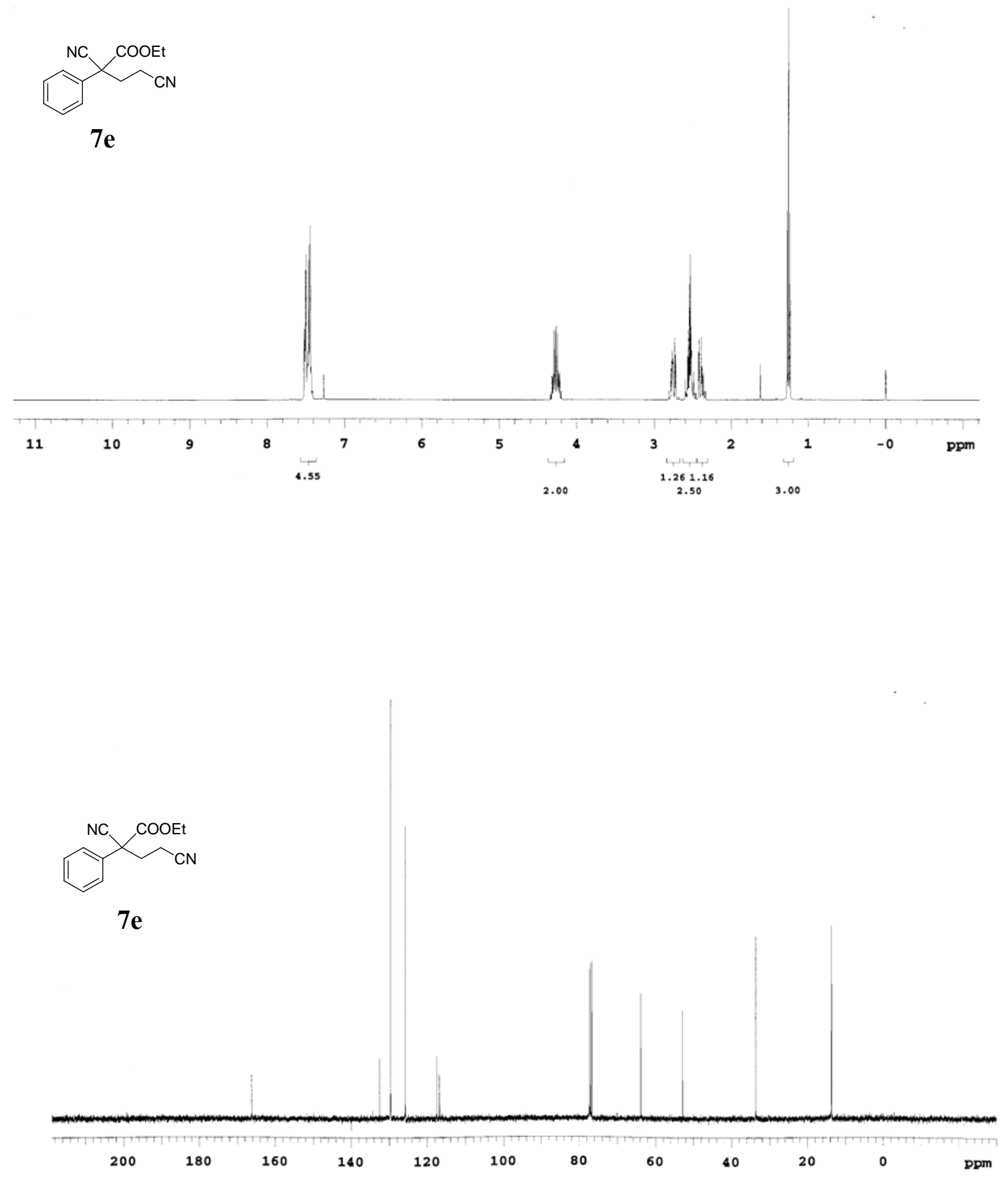

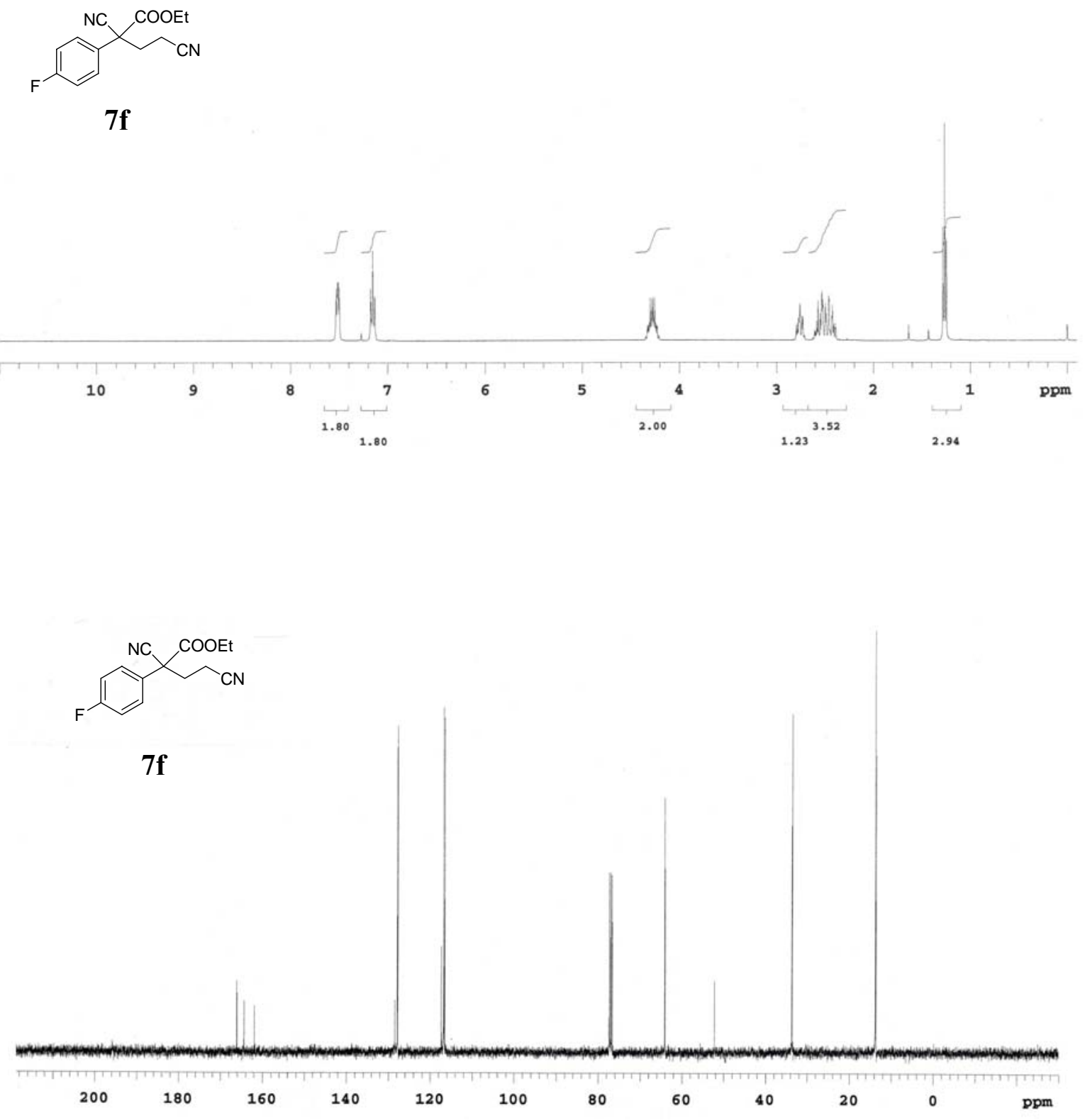

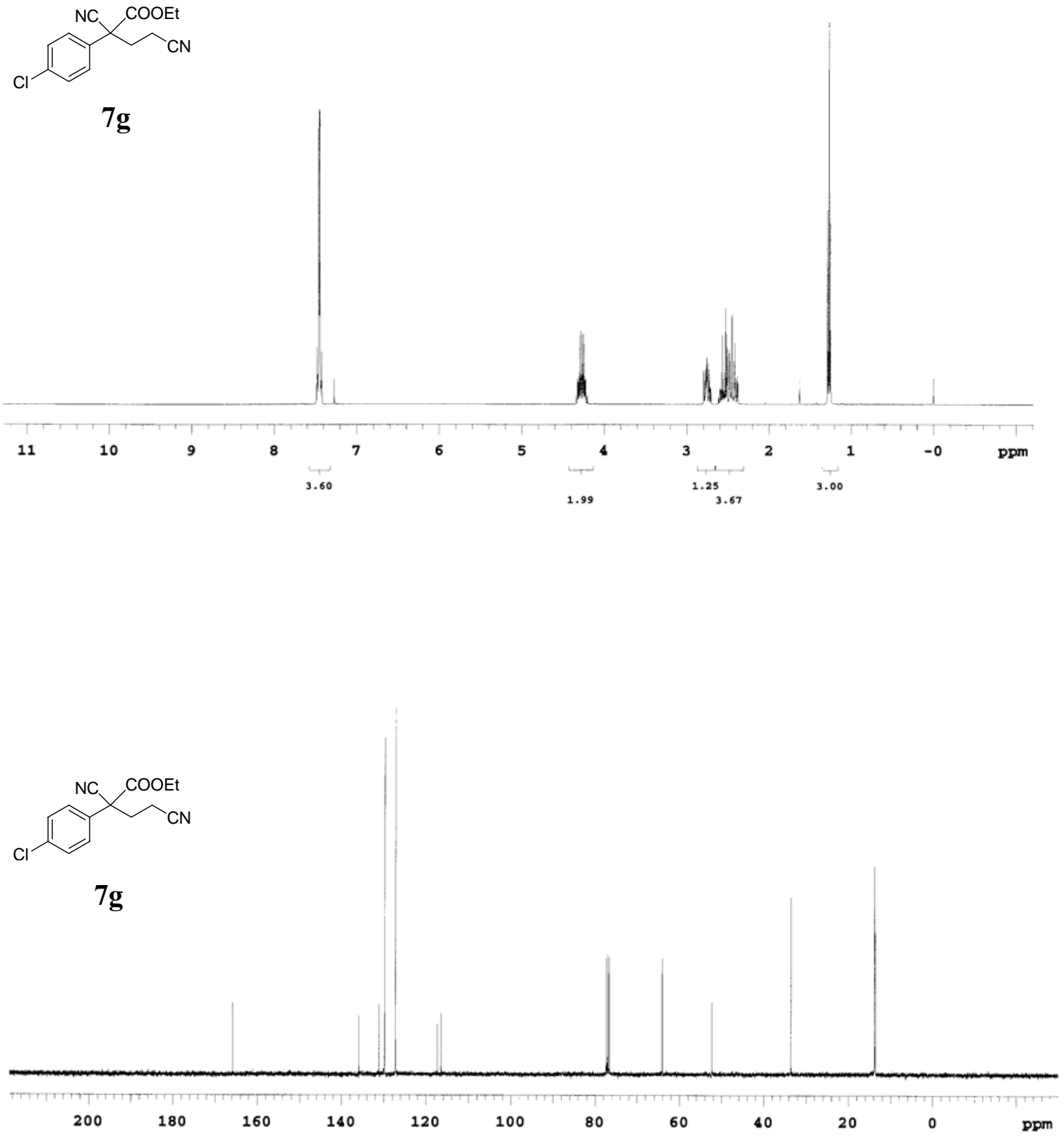

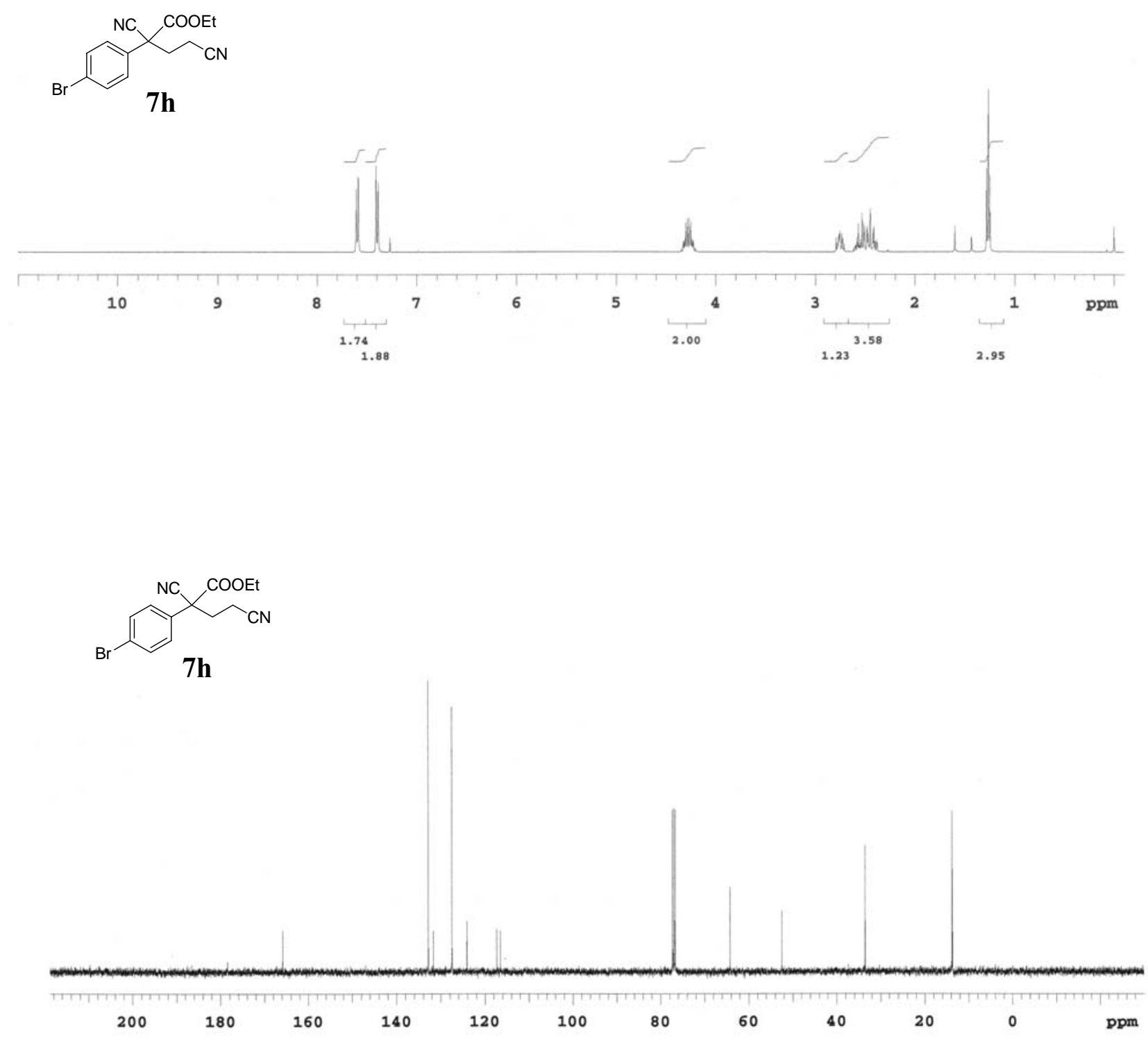

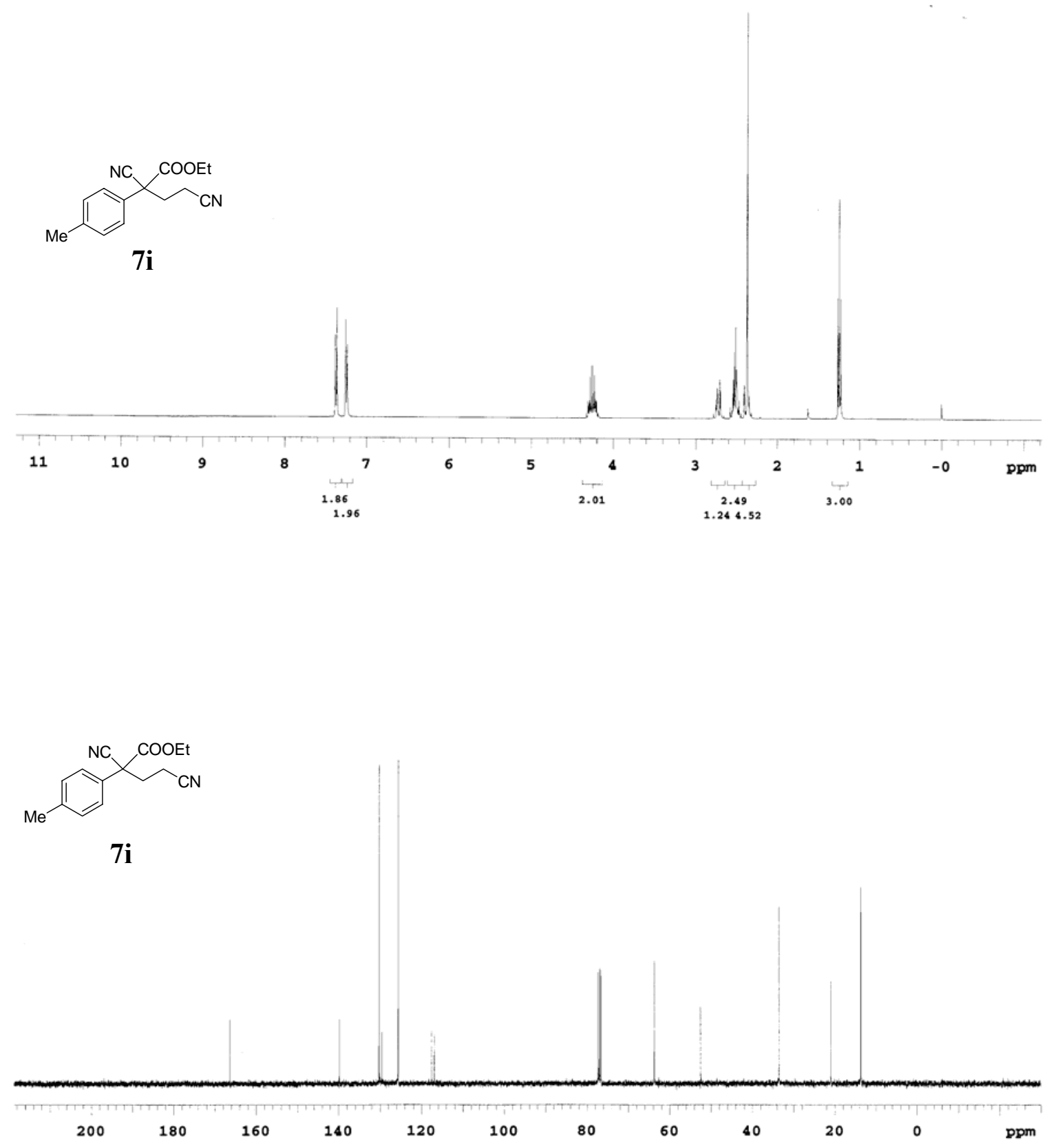

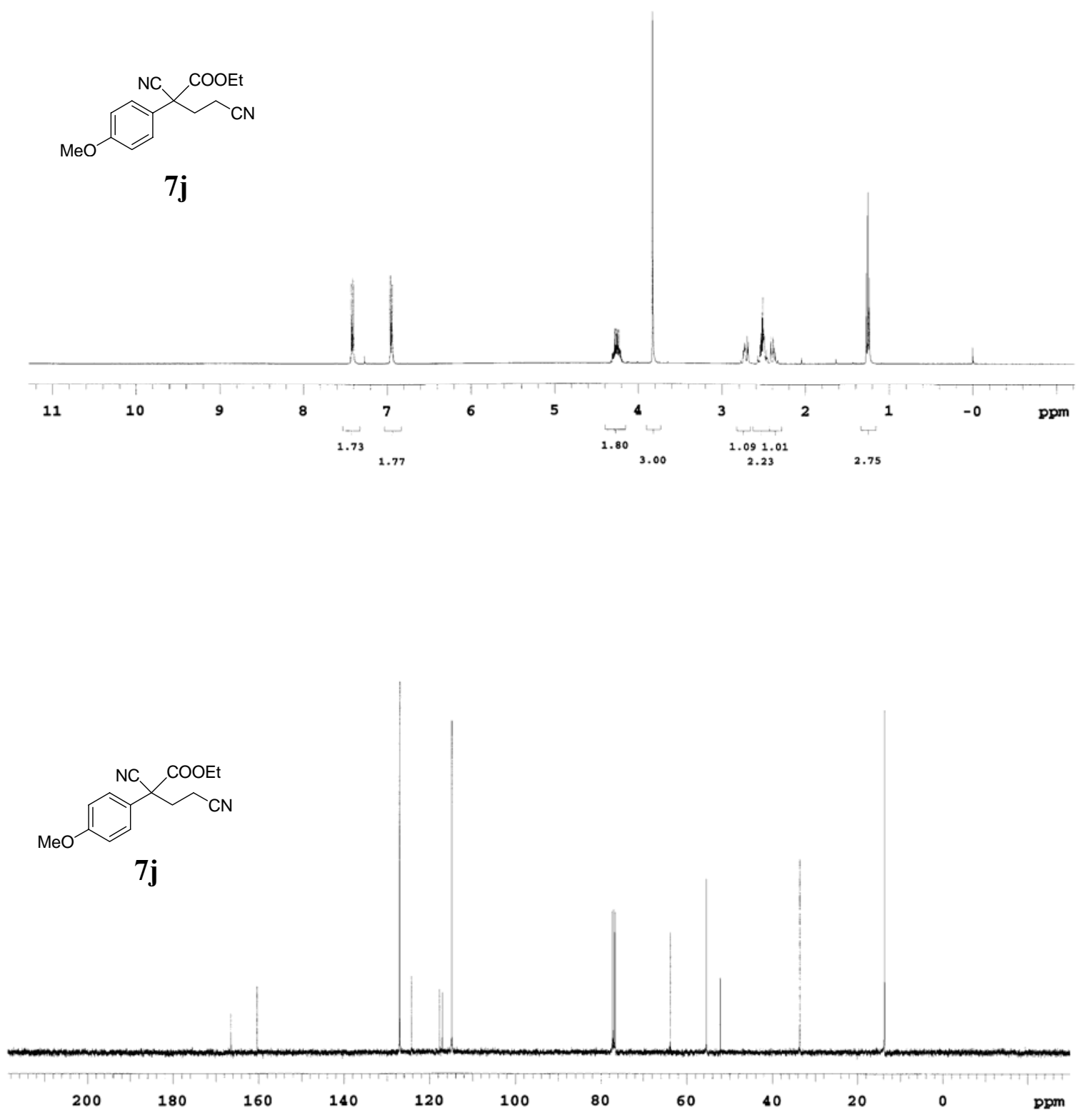

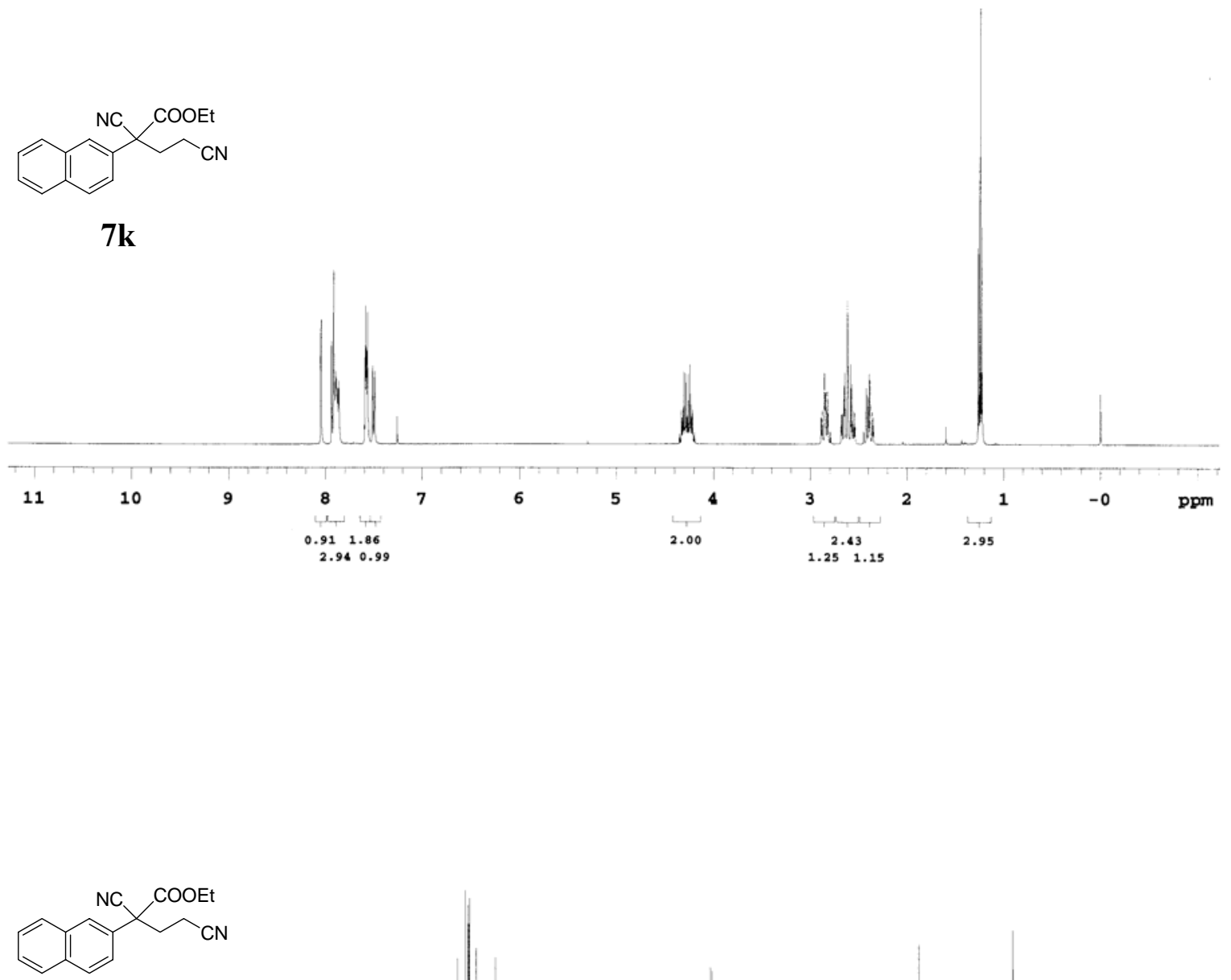

$7 k$

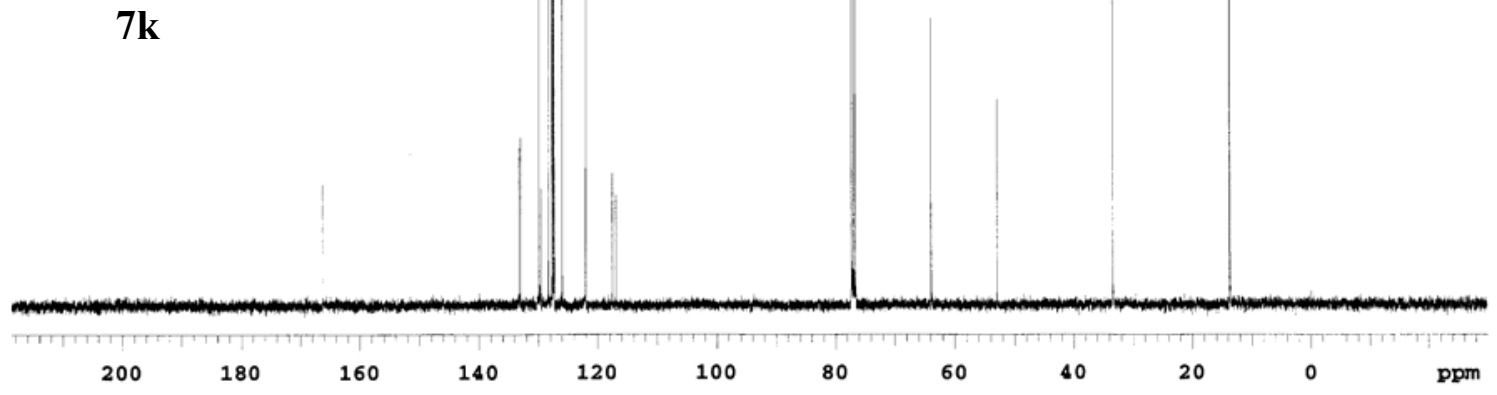



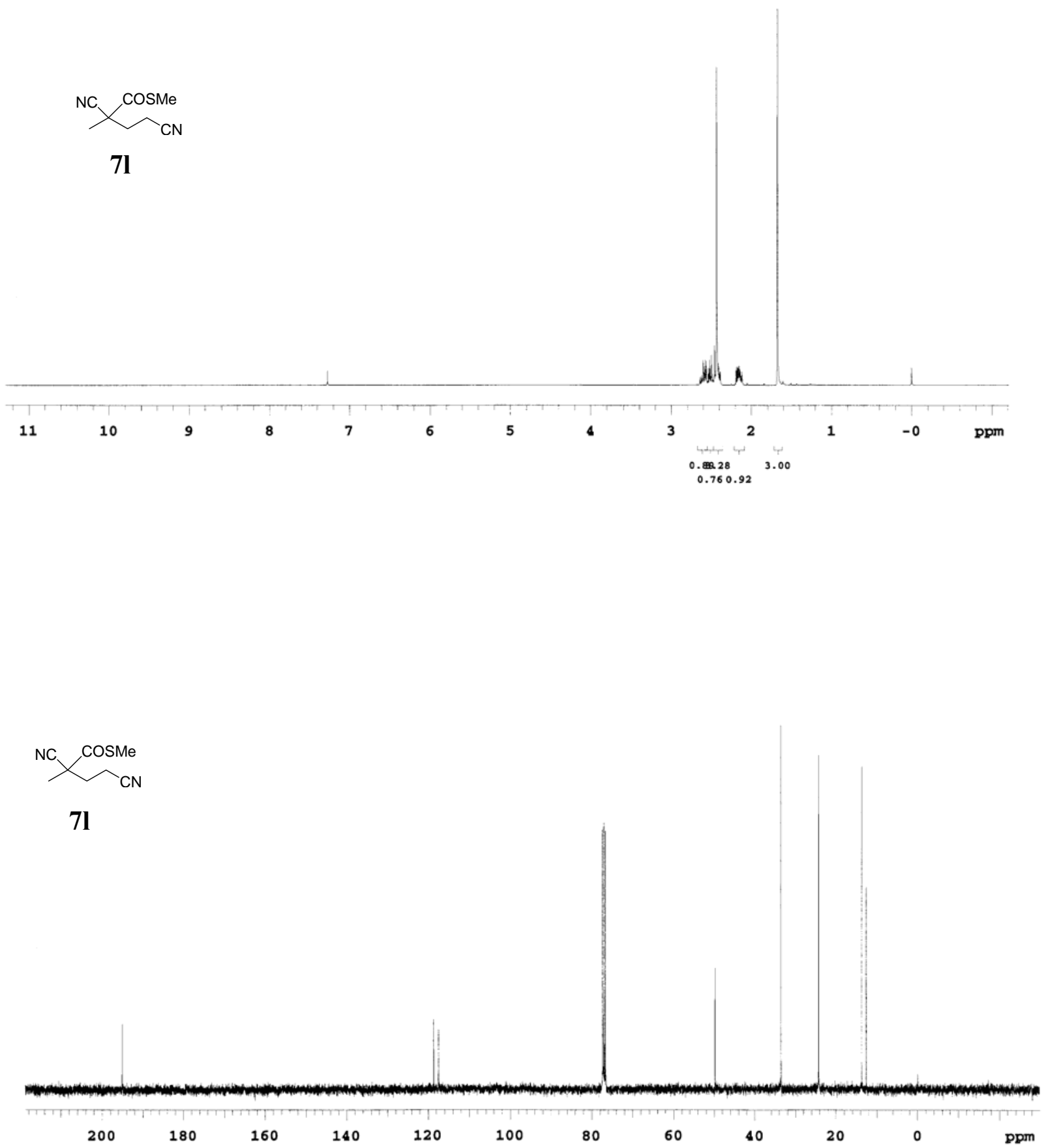

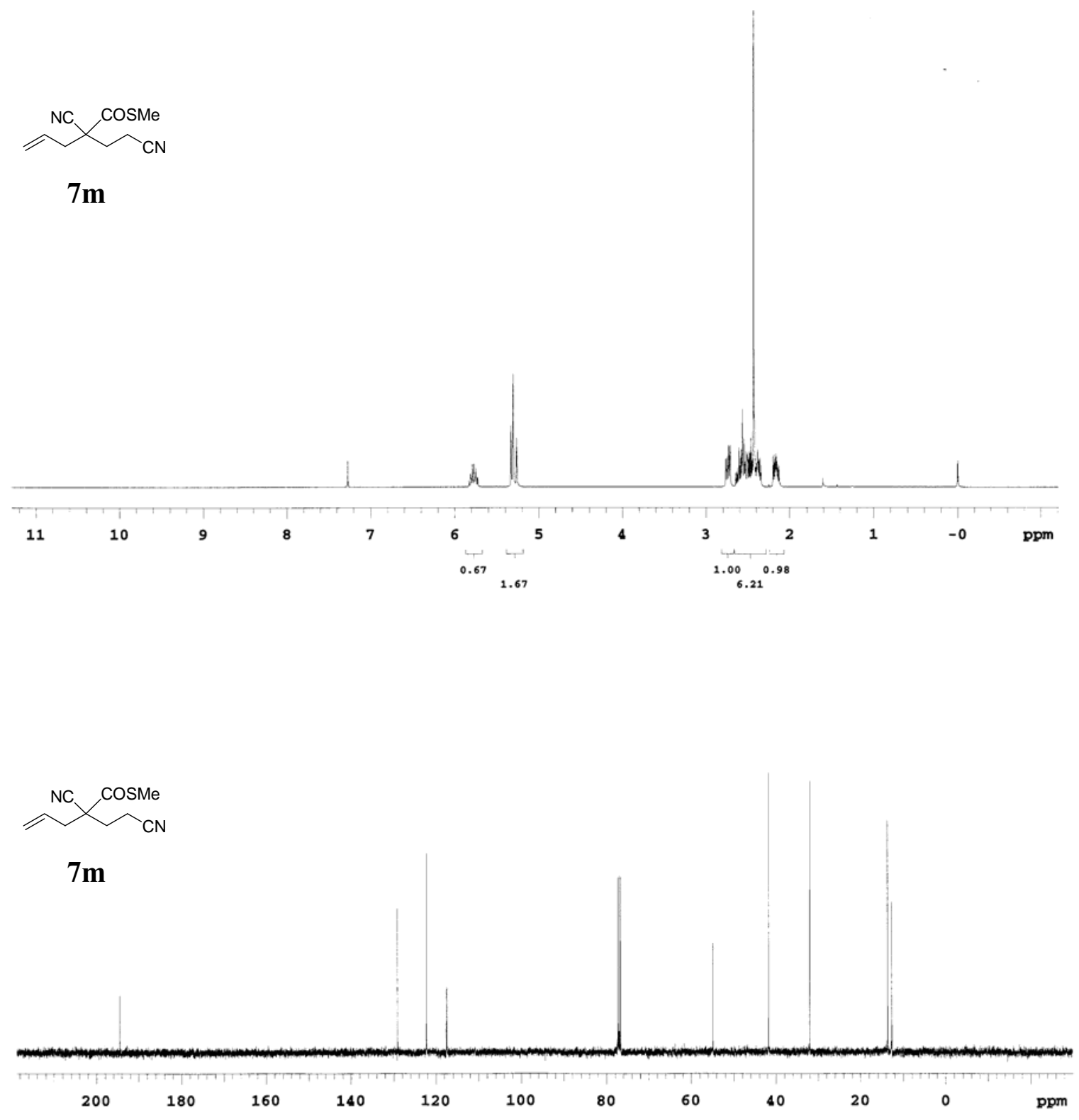

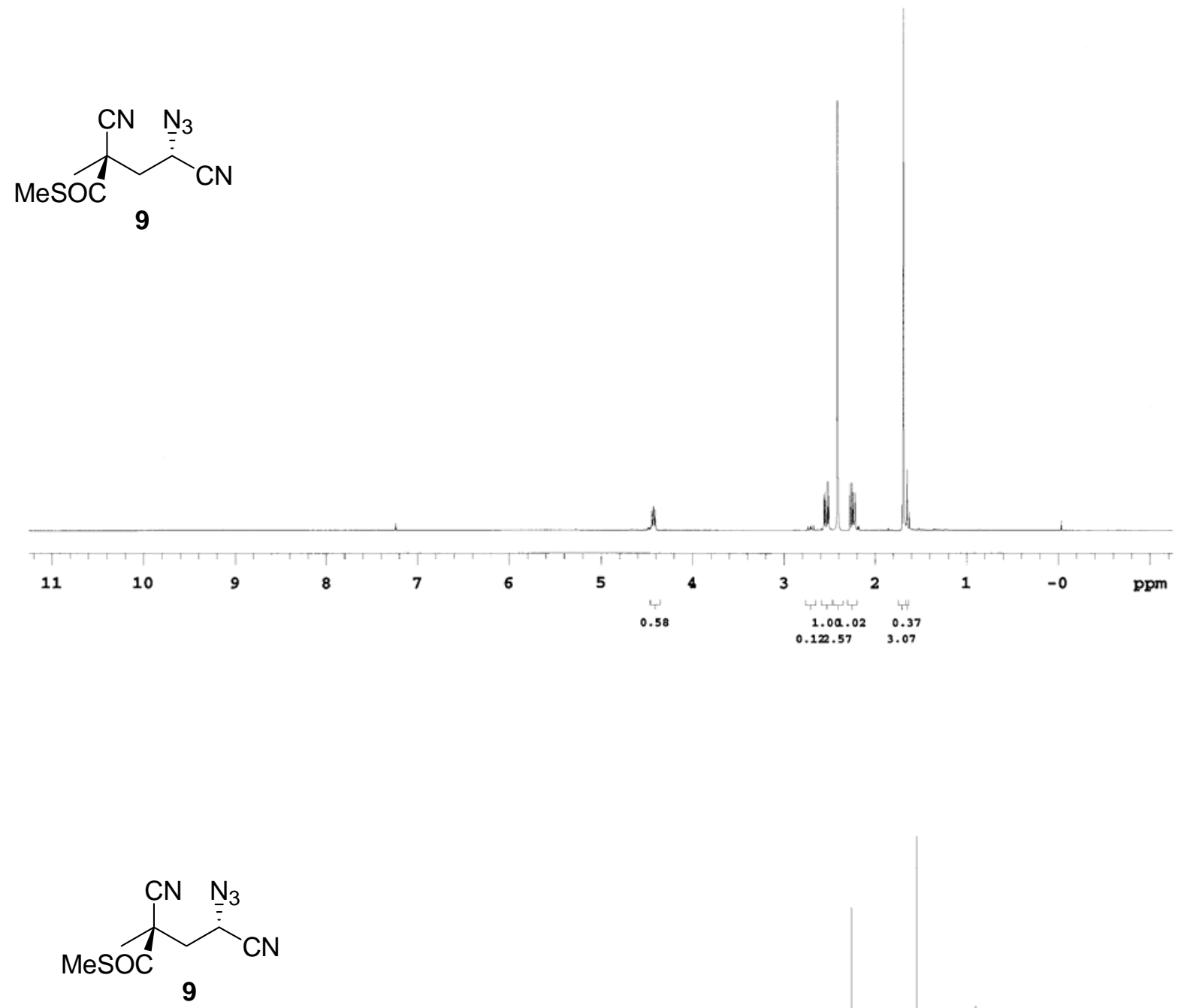

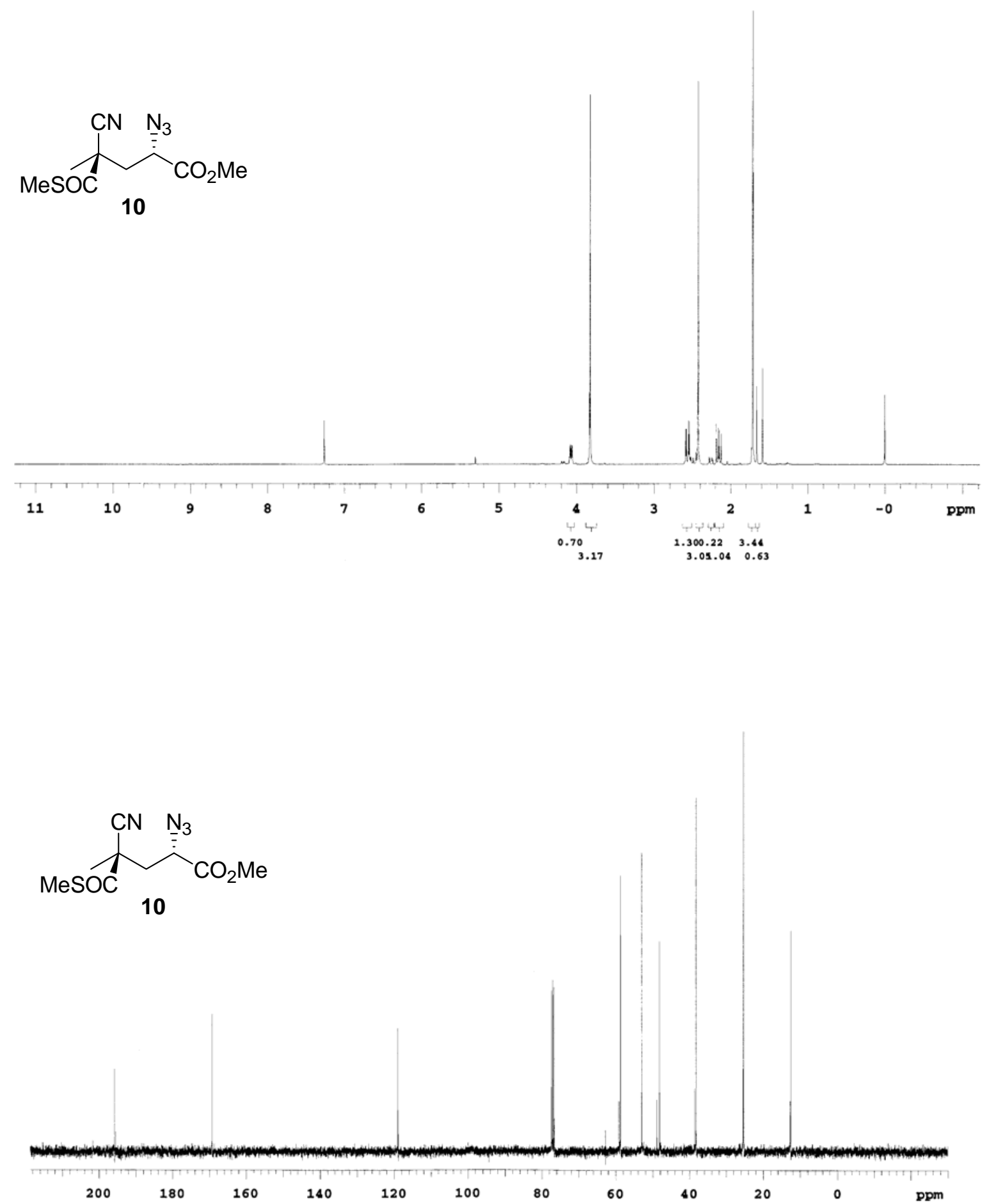
$\overbrace{\mathrm{OH}}^{\mathrm{CN}}$

11

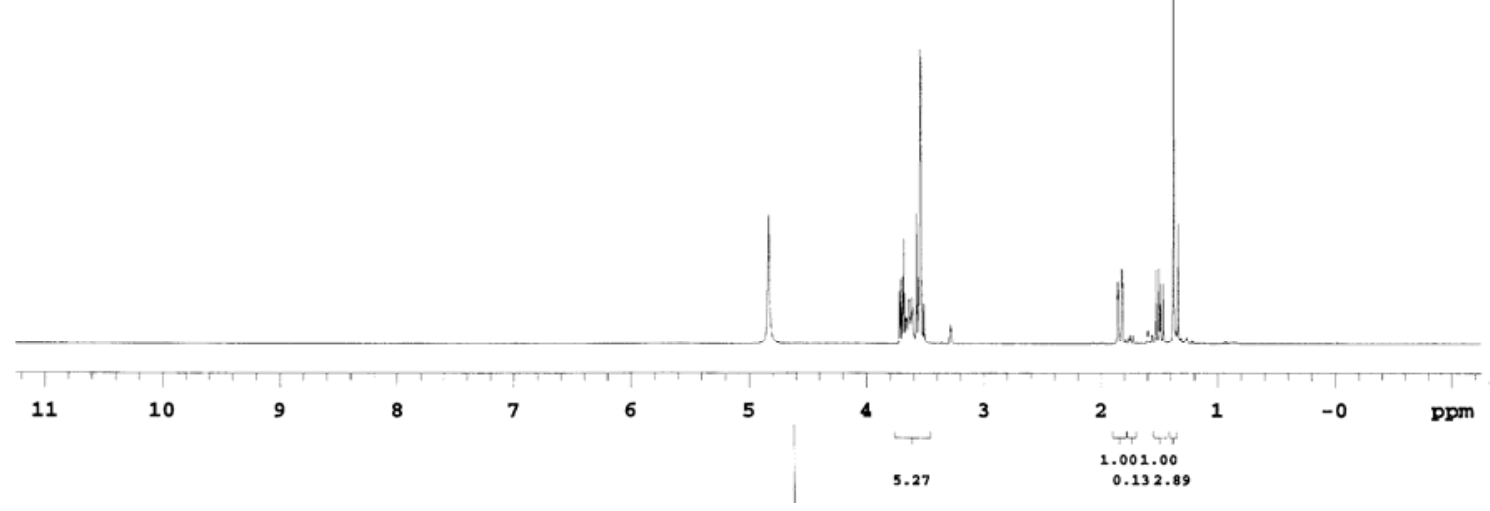<smiles>CC(C#N)(CO)CC([N])CO</smiles>

11

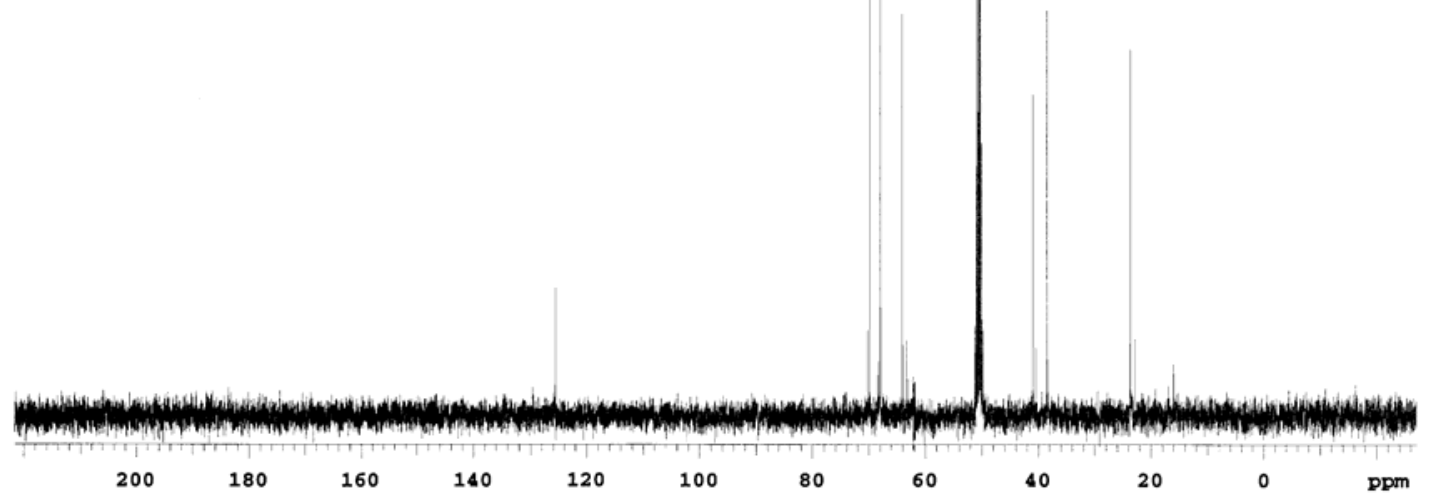


$\overbrace{\text { OTBDPS }}^{C N}$

12

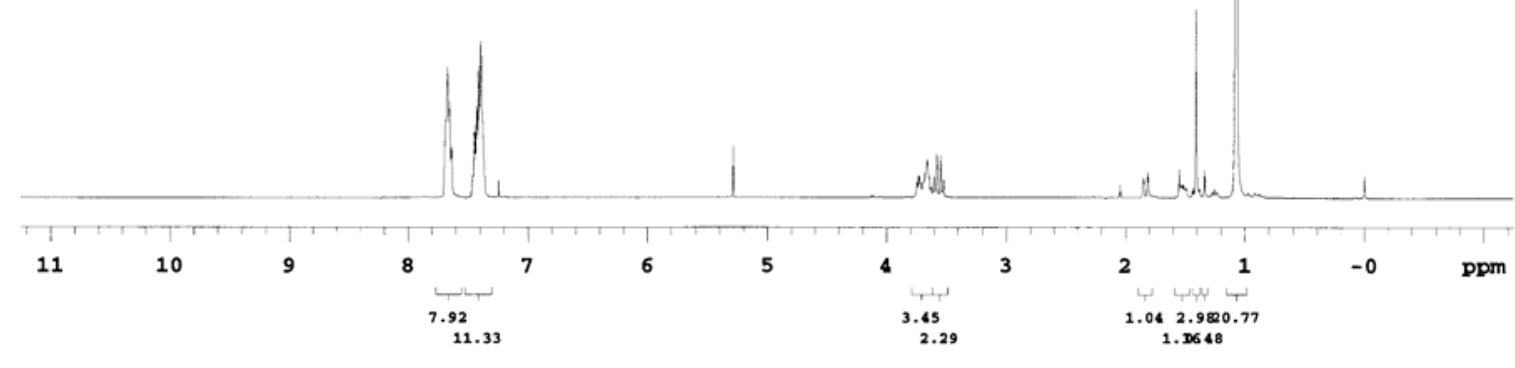

$\underbrace{C N}_{\text {OTBDPS }}$

12

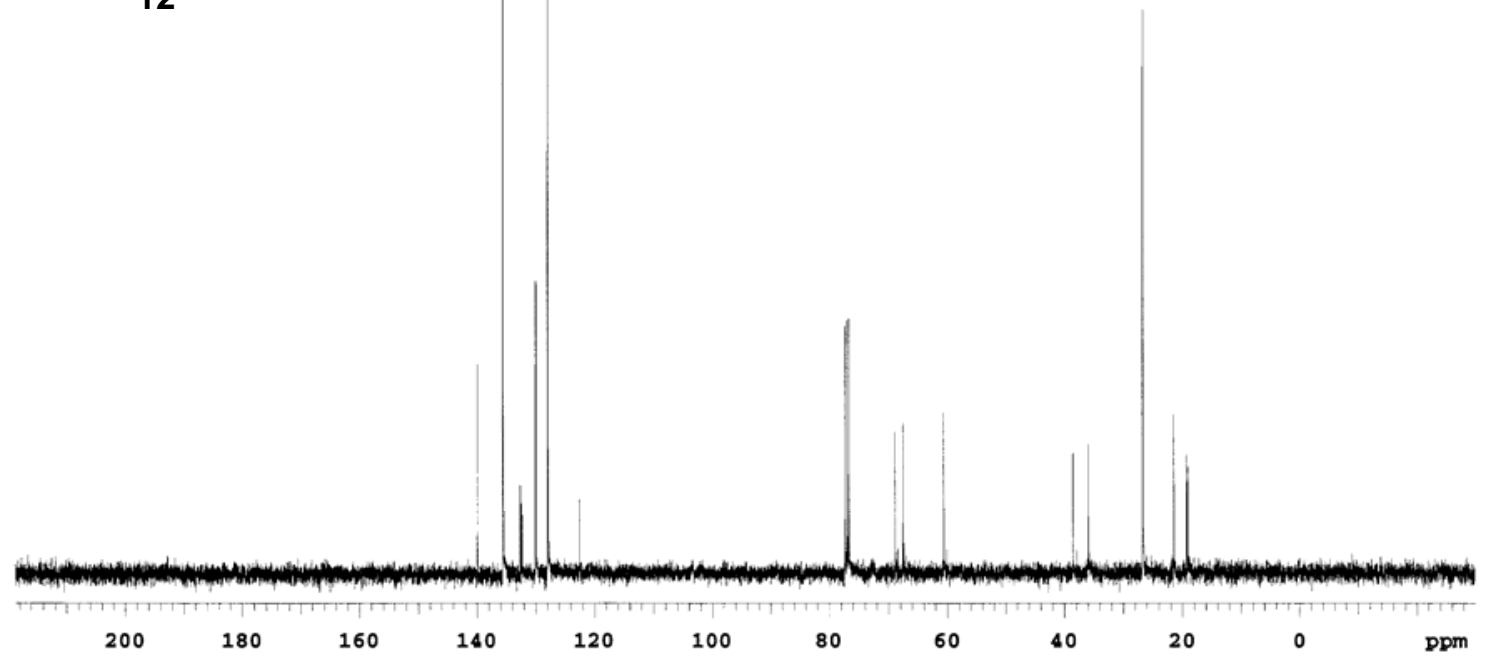


$\underbrace{\mathrm{H}_{2} \mathrm{NOCTBDPS}}_{\text {OTBDPS }}$

13

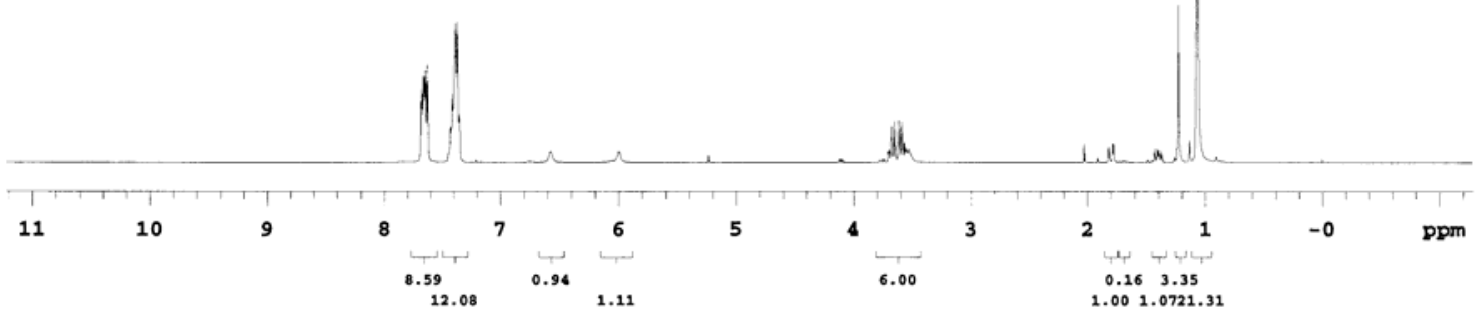

$\underbrace{\mathrm{H}_{2} \mathrm{NOCTBDPS}}_{\text {OTBDPS }}$

13

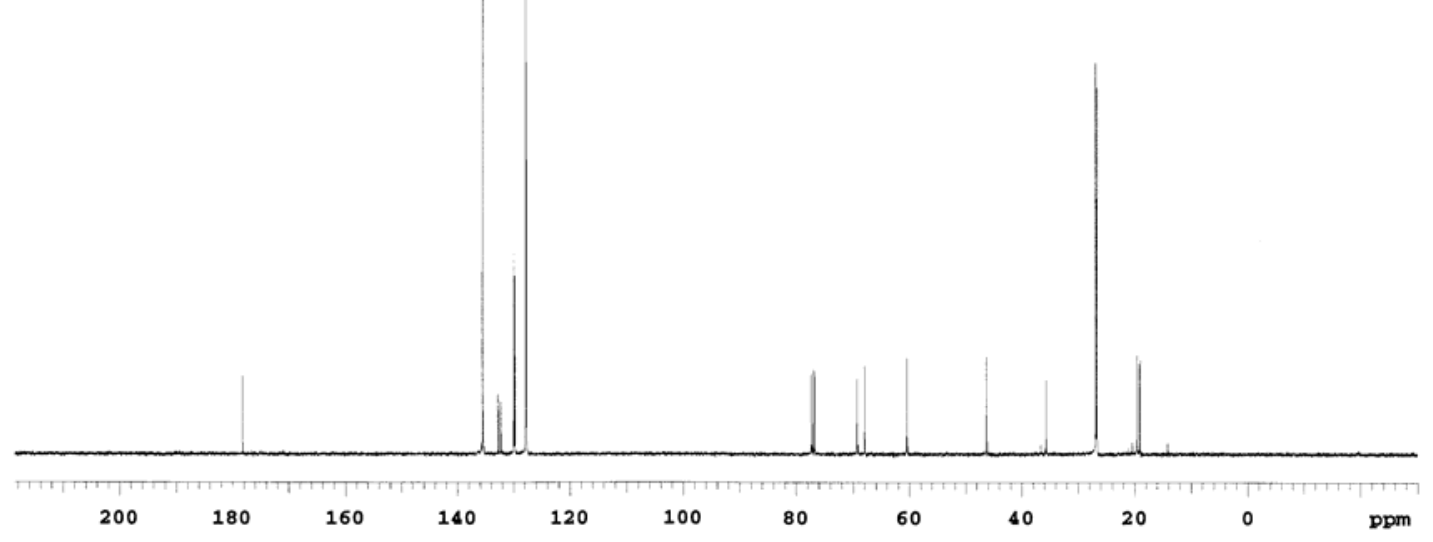



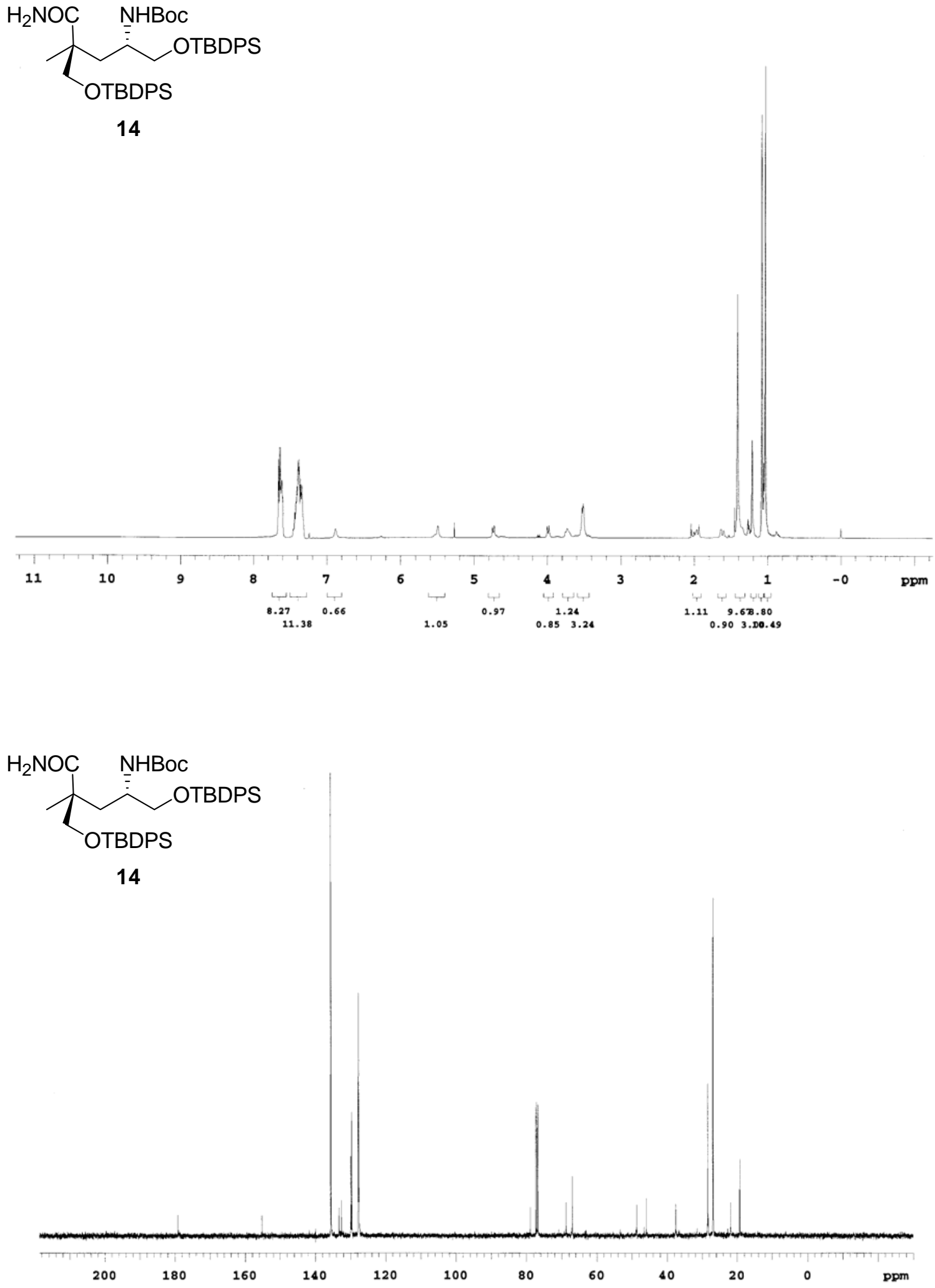


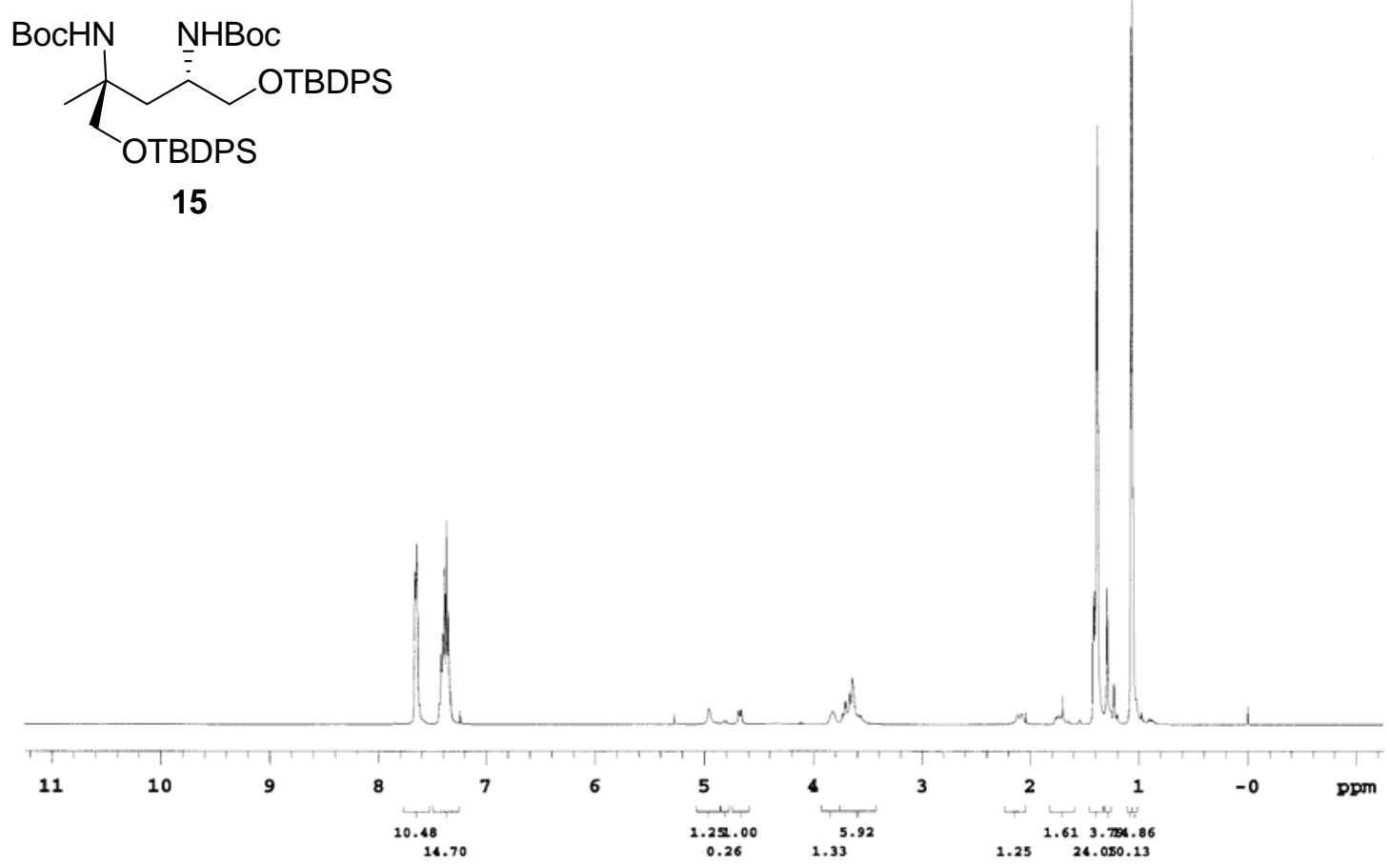

$\underbrace{\text { NHBoc }}_{\text {OTBDPS }}$

15

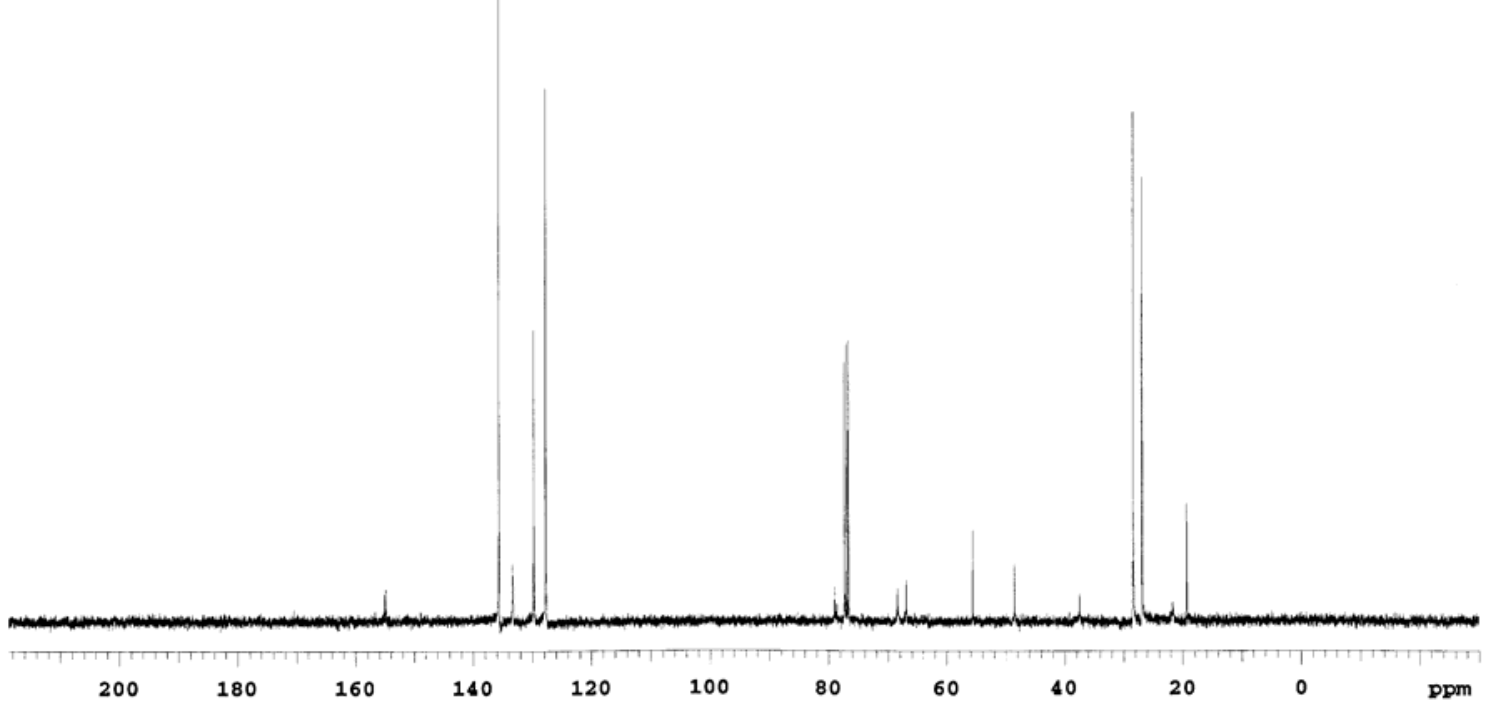



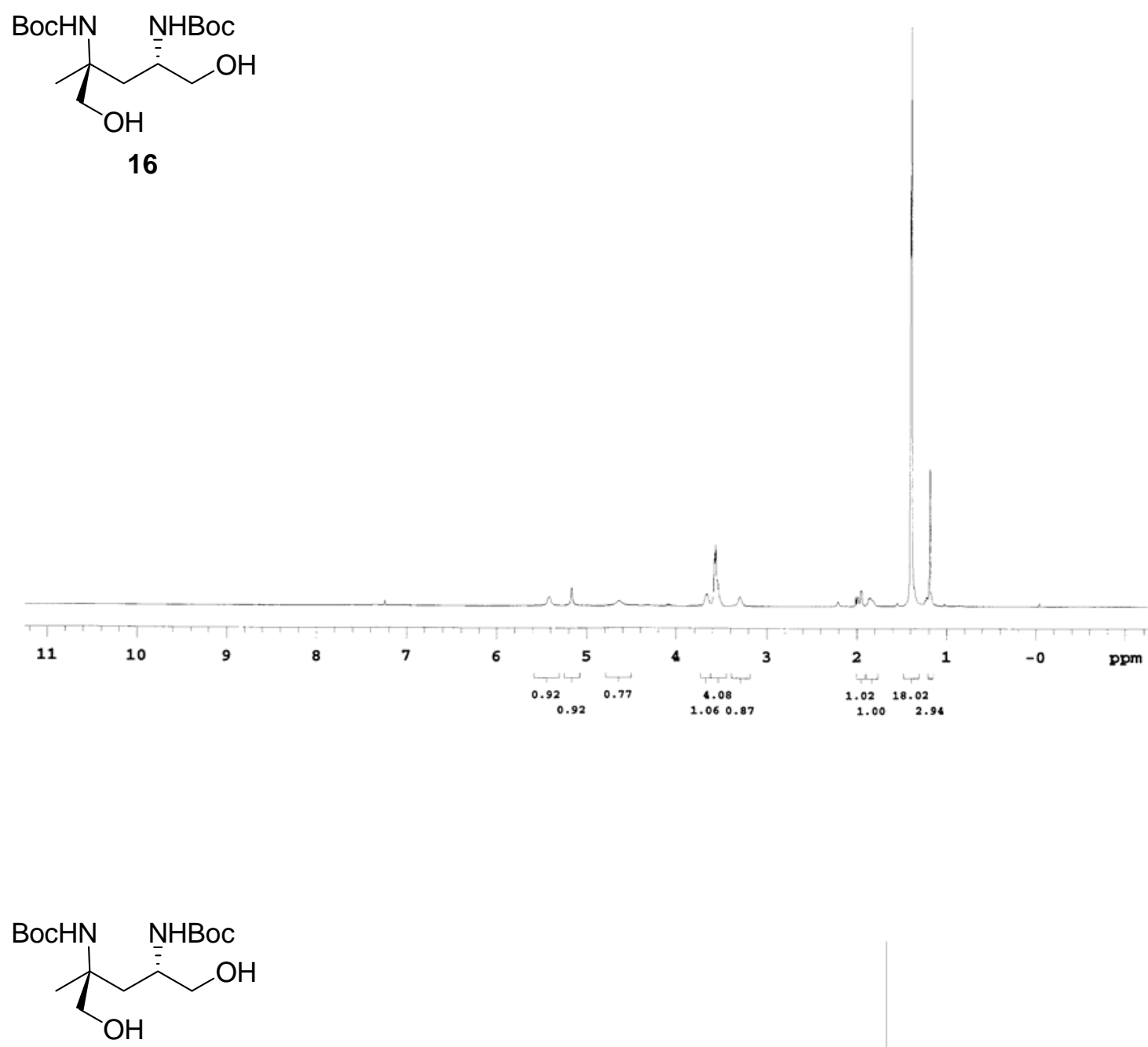

16

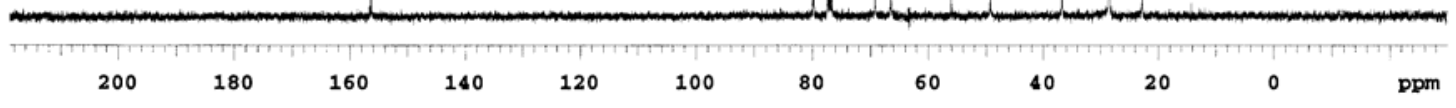



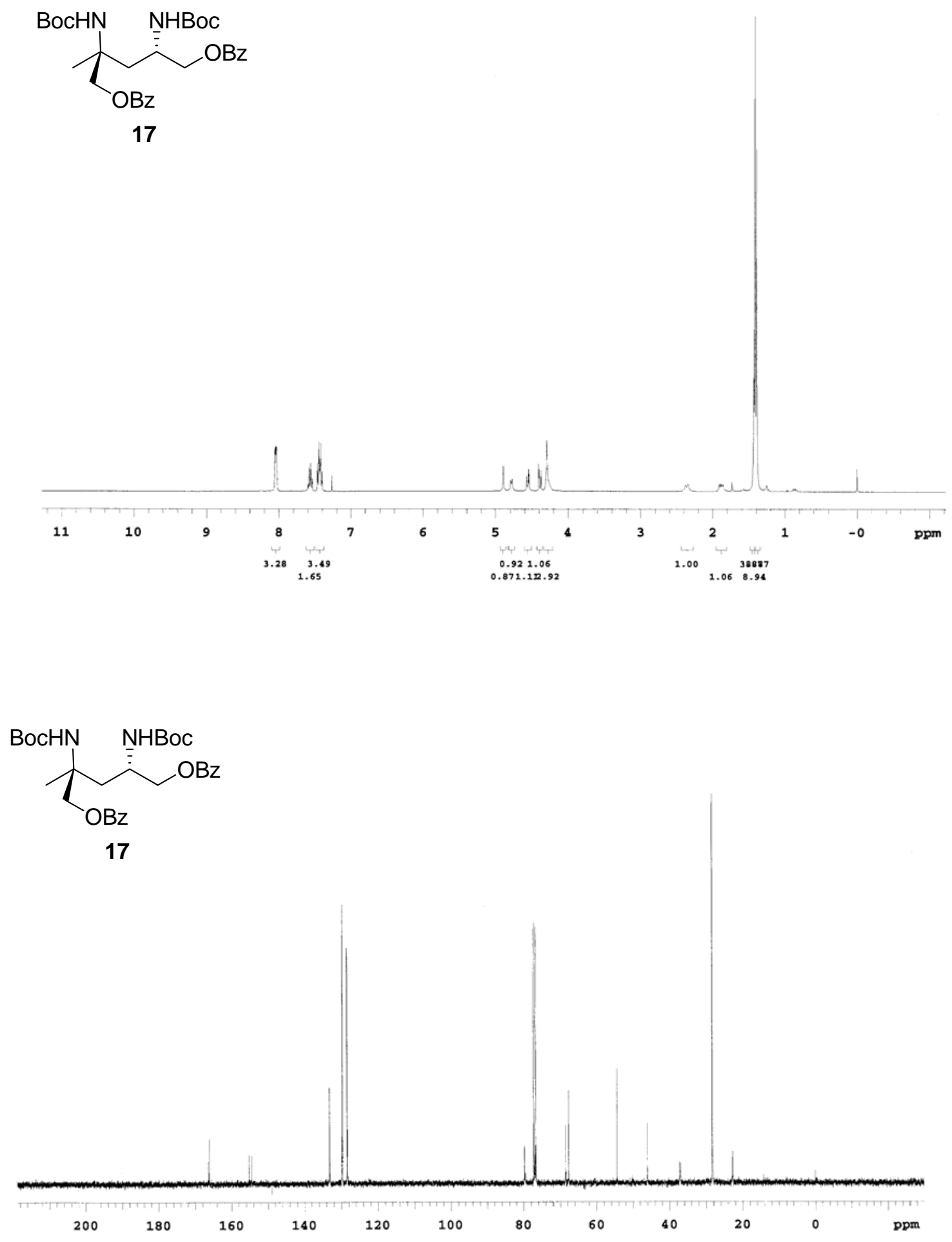
HPLC conditions for ee determination: Chiralcel OD, hexanes: isopropanol = 97: 3, 1.0 $\mathrm{mL} / \mathrm{min}, 220 \mathrm{~nm}$.

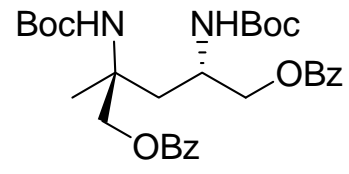

Racemic-17

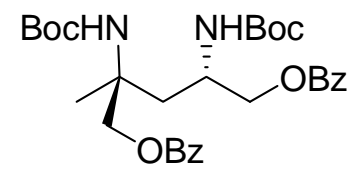

17
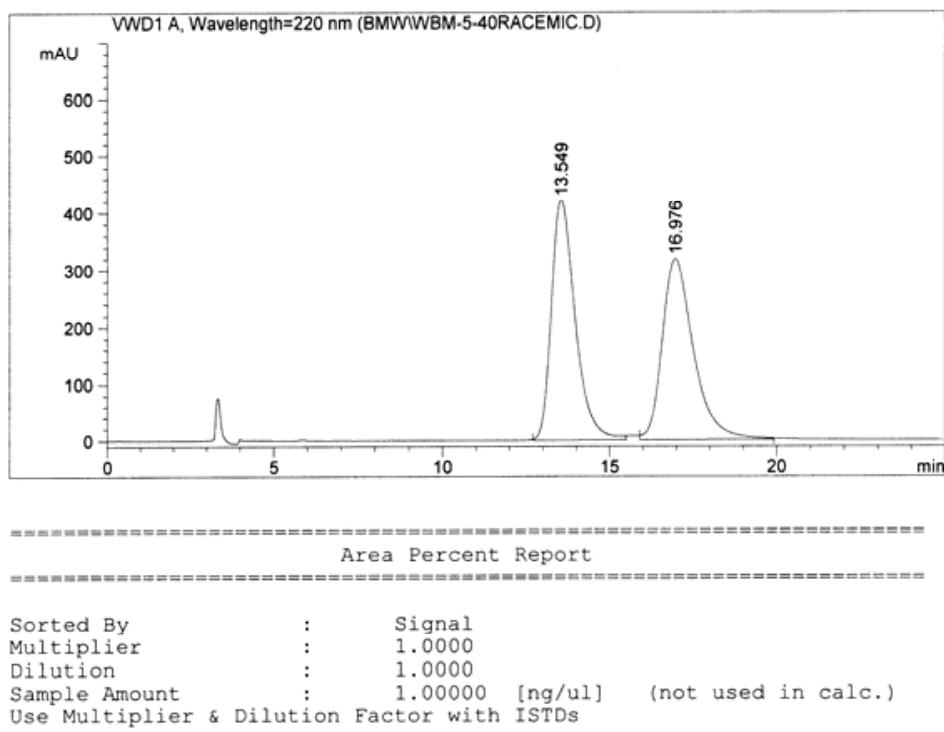

Use Multiplier \& Dilution Factor with ISTDs

Signal 1: VWD1 A, Wavelength=220 nm

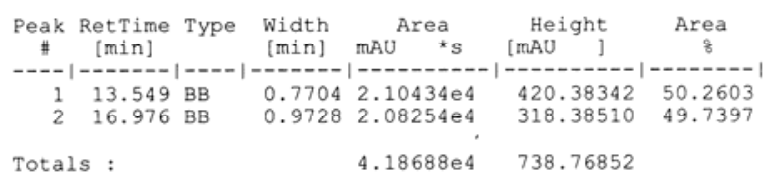

$\begin{array}{lll}\text { Totals : } & 4.18688 \mathrm{e} 4 & 738.76852\end{array}$

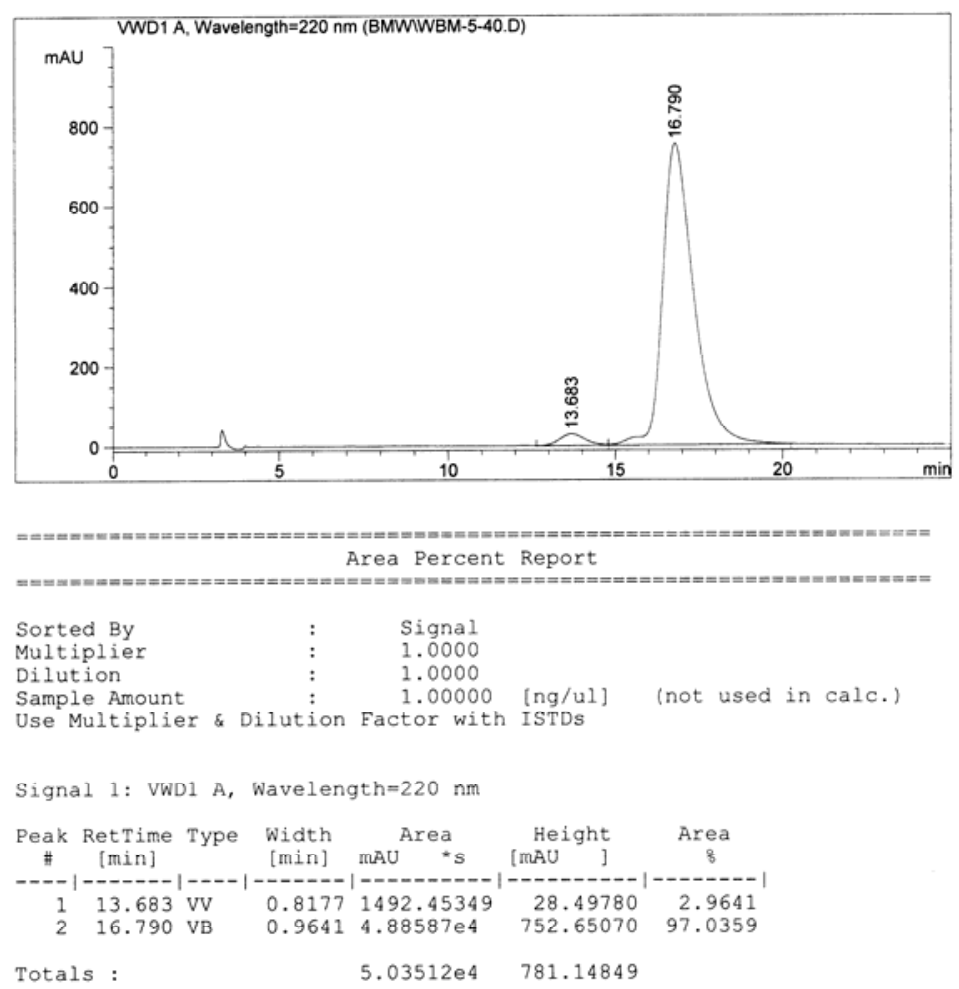

